\title{
Quantum linear Boltzmann equation
}

\author{
Bassano Vacchini ${ }^{a}$, Klaus Hornberger ${ }^{b}$ \\ ${ }^{a}$ Università degli Studi di Milano, Dipartimento di Fisica and INFN Sezione di Milano, Via Celoria 16, 20133 Milano, Italy \\ b Arnold Sommerfeld Center for Theoretical Physics, Ludwig-Maximilians-Universität München, Theresienstraße 37, \\ 80333 Munich, Germany
}

\begin{abstract}
We review the quantum version of the linear Boltzmann equation, which describes in a non-perturbative fashion, by means of scattering theory, how the quantum motion of a single test particle is affected by collisions with an ideal background gas. A heuristic derivation of this Lindblad master equation is presented, based on the requirement of translation-covariance and on the relation to the classical linear Boltzmann equation. After analyzing its general symmetry properties and the associated relaxation dynamics, we discuss a quantum Monte Carlo method for its numerical solution. We then review important limiting forms of the quantum linear Boltzmann equation, such as the case of quantum Brownian motion and pure collisional decoherence, as well as the application to matter wave optics. Finally, we point to the incorporation of quantum degeneracies and self-interactions in the gas by relating the equation to the dynamic structure factor of the ambient medium, and we provide an extension of the equation to include internal degrees of freedom.
\end{abstract}

Key words: Quantum Boltzmann equation, Quantum Brownian motion, Collisional decoherence, Atomic index of refraction, Dynamic structure factor

PACS: 03.65.Yz, 05.20.Dd, 03.75.-b, 47.45.Ab

published in: Physics Reports 478 (2009) 71-120

doi:10.1016/j.physrep.2009.06.001

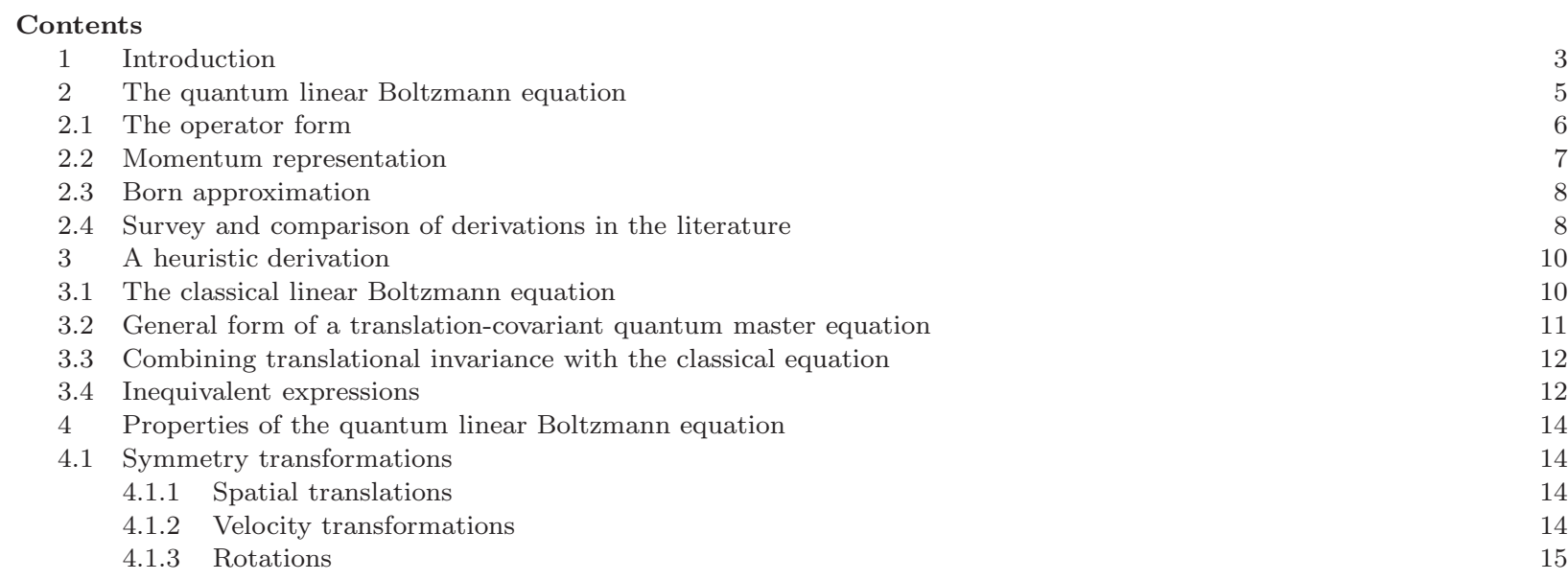

Preprint submitted to Elsevier

28 May 2018 
4.2 Approach to equilibrium

4.2 Detailed balance and the stationary solution

4.2.2 Entropy growth

4.3 Relaxation dynamics

4.3.1 Momentum and energy relaxation

4.3.2 Simulation algorithm

4.3.3 The loss term

4.3.4 Simulating the momentum and energy dynamics

5 The limit of quantum Brownian motion

5.1 The classical Kramers equation

5.2 The quantum Brownian limit

5.2.1 Conditions of validity

5.2.2 Diffusive limit of the quantum linear Boltzmann equation

5.2.3 Microscopic expression for the friction constant

5.3 The master equation of quantum Brownian motion

5.3.1 The diffusion coefficients and complete positivity

5.3.2 The quantum diffusion term

$6 \quad$ Matter wave optics in gaseous samples

6.1 The gas induced energy shift

6.2 The optical potential

6.3 Index of refraction

$7 \quad$ Collisional decoherence of matter waves

7.1 The limit of a massive tracer particle

7.2 Decoherence in position

7.2.1 The exact solution of pure decoherence

7.2.2 Experimental tests

7.3 Decoherence in momentum

7.4 Decoherence in the quantum Brownian motion limit

7.4.1 Position representation

7.4.2 Momentum representation

7.4.3 Relevance of the quantum correction term

8 The dynamic structure factor

8.1 An alternative formulation of the linear Boltzmann equation

8.1.1 The classical expression

8.1.2 Generalization to the quantum case

8.2 Properties of the dynamic structure factor

8.2.1 Expression in terms of the density correlation function

8.2.2 Connection to the laboratory-frame scattering cross-section

8.2.3 Detailed balance and the stationary solution

8.2.4 Fluctuation-dissipation relationship

8.3 Extension to different reservoirs

8.4 Inclusion of internal degrees of freedom

9 Conclusion

A Classical formulation

A.1 The classical linear Boltzmann equation

A.2 Approach to equilibrium

A.3 The friction coefficient of classical Brownian motion

B List of symbols

References 


\section{Introduction}

The Boltzmann equation, as the basic equation of kinetic gas theory, belongs to the foundations of classical statistical mechanics. Apart from its great relevance for concrete applications, it is a paradigm for the description of classical systems far from the thermodynamic equilibrium and for the understanding of irreversibility and equilibration [1/2/3/45. A closely related equation is used if one wants to describe how the motion of a single distinguished test particle is affected by elastic collisions with an ideal, stationary background gas. In both situations one deals with the evolution of a probability distribution defined on the phase space of a single particle. In case of the Boltzmann equation this is the marginal one-particle distribution of a dilute gas, whose self-interaction gives rise to the nonlinear form of the integro-differential equation. There is no such self-interaction if an individual test particle is considered, such that the corresponding equation is linear, like the Liouville equation. Apart form this, the linear equation shares much of its structure and many basic properties with the original Boltzmann equation, and it is derived the same way using the Stosszahlansatz - it is thus aptly called the linear Boltzmann equation 2. This equation for the motion of a distinguished test particle has found important applications, especially in the study of transport phenomena 6[7].

The present review is devoted to the quantum analog of the linear Boltzmann equation. It is therefore important not to confuse its classical counterpart with a "linearized Boltzmann equation". The latter is an approximation of the original Boltzmann equation for a self-interacting gas, which is valid when the gas is close to equilibrium and the interactions are a weak perturbation. In the linear Boltzmann equation, in contrast, the coupling to the gas can be strong and the distinguished test particle may be very far from an equilibrium state. The background gas, on the other hand, is taken to be in a stationary state, typically at thermal equilibrium, which remains undisturbed by the presence of the test particle.

In the quantum physics literature a large number of master equations has been termed "quantum Boltzmann equation". The name is likely to appear whenever the dynamics is amenable to a kinetic description based on interaction events that can be described as scattering processes. A first basic result on a quantum extension of the original Boltzmann equation was the introduction of a correction accounting for the effects of quantum statistics in the classical collision term 8 8910. Moreover, Boltzmann-like kinetic equations appear in a wealth of situations with the aim of describing relaxation and transport properties in a quantum setting. Most recently, the study of quantum kinetic equations required for the description of degenerate Bose gases has been the object of extensive research, see, e.g., the series of papers [11]12[13 14 15[16]17] and references therein.

In the field of mathematical physics, major efforts have been undertaken in order to rigorously derive a quantum version of the nonlinear Boltzmann equation. Here, one typically starts out from the many-particle Hamiltonian with a simple interaction potential and seeks to obtain a nonlinear equation of motion for the reduced single-particle state by considering approximations for the hierarchy of equations of motion of many-particle distribution functions, see [18|19|20|21|22|23] for reviews on recent results. Similar attempts have been devoted to the derivation of the quantum linear Boltzmann equation, using a random potential approach, see 24|25|26] for recent results. However, it should be emphasized that even the classical Boltzmann equation has not yet been properly derived in the rigorous sense of mathematical physics. The problem is substantially more difficult in the quantum formulation, due to the need for accommodating the unitarity of the underlying many-body dynamics and due to the quantum restrictions of the associated phase-space representation.

The situation is more tractable if one asks for the proper quantum version of the linear Boltzmann equation. Due to the inherent linearity of the problem, the quantum equation of motion must be given by a linear mapping of the statistical operator representing the motional state of the test particle. It can be expressed in the framework of quantum dynamical semigroups 27/28 29] since the use of a Boltzmann equation implies that the time evolution is Markovian. It follows that the generator of the irreversible time evolution must have the Gorini-Kossakowski-Sudarshan-Lindblad form [30|31; this guarantees complete positivity, thus ensuring that the motion of the reduced single particle state is compatible with an underlying many particle state that evolves in a unitary fashion. 


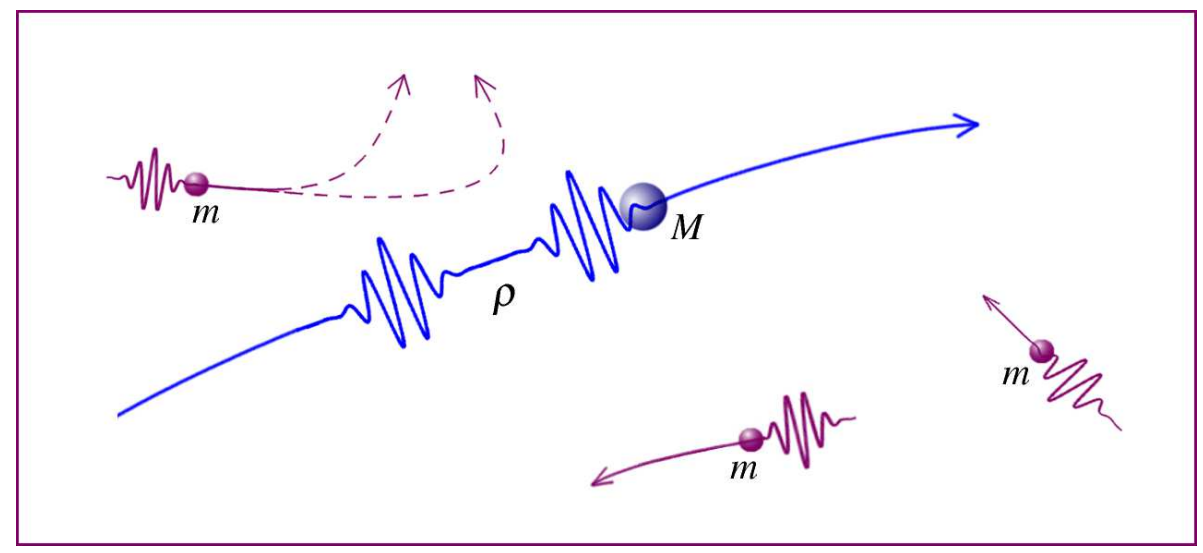

Fig. 1.1. (Color online) The quantum linear Boltzmann equation describes the motion of a single, distinguished test particle in an ambient medium, taken to be an ideal, stationary, and uniform background gas. In the framework of the Markov approximation, it is valid for arbitrary initial states of motion, such as the non-classical spatial superpositions found in an interferometer. As such it describes the interplay of decoherence phenomena at short time scales with the dissipative effects and the thermalizing behavior expected in the longer-term. The interaction with the gas is accounted for in a non-perturbative and microscopic fashion, by means of the exact two-body scattering amplitudes. The equation ceases to be applicable if the neglect of three-body collisions or correlated scattering events cannot be justified, as in the case of liquids.

Using the framework of Lindblad master equations, the natural quantum counterpart of the classical linear Boltzmann equation can indeed be obtained as a unique master equation. It thus describes how the free quantum motion of a test particle, possibly in a very non-classical, delocalized state, is modified by the presence of a background gas, see Fig. 1.1. As with the classical linear Boltzmann equation, the quantum master equation applies provided (i) the background gas is sufficiently dilute, such that three-body collisions can be neglected, (ii) the particle interaction is sufficiently short-ranged, such that scattering theory can be applied, and (iii) the gas temperature is sufficiently high, such that effects of quantum statistics can be disregarded and the Markov assumption is justified. Both the classical and the quantum formulation incorporate the two-body interaction between test particle and a gas molecule in a non-perturbative fashion by means of scattering theory. While the classical equation contains the differential cross-section to account for the molecular collisions, the quantum master equation involves pairs of scattering amplitudes. These objects, which feature a complex phase, show up as operator-valued quantities if the master equation is stated in a representation independent form.

The main significance of the quantum linear Boltzmann equation lies in the fact that it serves to describe the interplay of two distinct gas-induced phenomena, typically occurring on different time scales and usually requiring a non-perturbative description of the coupling to the gas. On one hand, this is the collisional decoherence effect, taking place at short times when the energy exchange between test particle and medium can be disregarded. On the other hand, the test particle experiences friction, leading on a longer time scale to a slowing down of the particle and its eventual thermalization. The equation should thus predict how a test particle, starting out in a delocalized state, gradually loses its ability to display quantum interference, due to the dissemination of position information effected by the gas collisions. One then expects the quantum particle, whose motional state is increasingly hard to distinguish from a classical one, to display the relaxation and equilibration behavior predicted by the classical linear Boltzmann equation. It is thus an important consistency requirement that the quantum linear Boltzmann equation indeed reduces to the classical version once the quantum state of motion is indistinguishable from a classical state. Also in the other relevant limiting cases the equation turns into well-established forms, in particular in the limit of quantum Brownian motion, of pure collisional decoherence, and of coherent forward scattering, as will be discussed below.

In the present review we do not aim at providing a microscopic derivation of the quantum linear Boltzmann equation; such a calculation was given recently in some detail in 32 . Instead, we will motivate the form of the equation in a more succinct line of reasoning by combining natural physical and mathematical requirements. It builds on the structure of the classical equation and on Holevo's characterization of general translation- 
covariant quantum dynamical semigroups [33], by exploiting both the required limiting expression of the equation and the translational symmetry expected in a homogeneous medium. Apart from the operator form, we will also discuss the equation and its limiting cases in different representations, each admitting specific physical insights.

The physics described by the quantum linear Boltzmann equation is discussed and applied in quite different fields of physics, ranging from mathematical physics and statistical mechanics to matter wave optics. Traditionally, quantum versions of the linear Boltzmann equation were introduced in order to provide a microscopic explanation of transport phenomena, or to obtain a derivation of quantum Brownian motion. These are situations where the relevant quantum states are close to the state of motion of a classical particle.

Only recently it has become possible to study the influence of a gas environment on the motion of a molecule which is very far from a classical state, such as in a superposition of macroscopically distinct spatial positions 34. This is a result of the experimental advances in the interferometry of massive particles [3536], allowing one to observe the phenomenon of decoherence independently from dissipative effects. In fact, the main experimental applications and confirmations of the quantum linear Boltzmann equation are found in situations where the quantum coherences described by the statistical operator play a crucial role. This applies to the study of the refractive index for matter waves traveling through a gas [3738, and in particular to the analysis of collisional decoherence for massive particles [39]. It is worth noting that the seminal paper on decoherence by Joos and Zeh [40, seeking to explain the absence of quantum delocalization in a dust particle by the scattering of photons and air molecules, derived and studied what the authors called a Boltzmann-type master equation. Two decades later, the long quest for the characterization of the phenomenon of collisional decoherence has now reached a mature theoretical description, permitting its quantitative experimental confirmation.

The article is organized as follows. In Sect. 2 we introduce the equation both in its operator form and in the momentum representation. Section 3 provides an intuitive line of derivation, based on reconciling the expression of the classical linear Boltzmann equation with the general form of a translation-covariant generator of a quantum dynamical semigroup. Various properties and features of the quantum linear Boltzmann equation are then discussed in the following sections, by first addressing phenomena with a classical counterpart, and then moving to problems which can only be considered in a quantum setting. Specifically, Sect. 4 considers the symmetry properties of the equation and its relaxation and equilibration behavior, while Sect. 5] is devoted to the limiting case of quantum Brownian motion, obtained if the test particle is much more massive than the gas particles. Section [6 deals with a distinguished quantum effect, the index of refraction experienced by a matter wave due to the presence of a background gas, which depends on the coherent part of the quantum linear Boltzmann equation. The phenomenon of decoherence is then addressed in Sect. 7. both regarding collisional decoherence and more general forms. Section 8 discusses an alternative expression of the quantum linear Boltzmann equation in terms of the dynamic structure factor of the gas, suggesting a generalization to the case of quantum degenerate background gases. It further provides the extension of the quantum linear Boltzmann equation to include internal degrees of freedom. We finally present our conclusions in Sect. 9] where we also point to possible future developments.

\section{The quantum linear Boltzmann equation}

We start by introducing the general expression of the quantum linear Boltzmann equation in its representationindependent operator form. In order to understand why the scattering amplitudes and distribution functions in this expression must be operator-valued, and why the momentum projections parallel and perpendicular to the momentum exchange play different roles, it will be helpful to represent it in the appropriate coordinates. We therefore proceed by presenting the equation in the momentum basis, which will also facilitate the comparison with the classical linear Boltzmann equation. We conclude this section with a discussion of the Born approximation and a brief outline of existing approaches for deriving the equation. 


\subsection{The operator form}

The quantum linear Boltzmann equation is a Markovian master equation for the statistical operator $\rho$ describing the motion of a distinguished test particle in a gas. It has the form

$$
\begin{aligned}
\frac{\mathrm{d}}{\mathrm{dt}} \rho & =\mathcal{M} \rho \\
& =\frac{1}{i \hbar}\left[\mathrm{H}_{0}+H_{\mathrm{n}}(\mathrm{P}), \rho\right]+\mathcal{L} \rho,
\end{aligned}
$$

where $\mathrm{H}_{0}=\mathrm{P}^{2} /(2 M)$ is the kinetic energy of the test particle and $H_{\mathrm{n}}(\mathrm{P})$ describes an energy shift due to the presence of the background gas, as defined in Eq. (2.5). Here and in the following we use sans-serif symbols to denote operators in a single particle Hilbert space; variables referring to the test particle will be set in upper-case letters.

The superoperator $\mathcal{L}$ in (2.1) is a linear mapping which accounts for the incoherent part of the collisional interaction with the gas. It can be expressed in Lindblad form as [4]

$$
\begin{aligned}
\mathcal{L} \rho= & \int \mathrm{d} \mathbf{Q} \int_{\mathbf{Q}^{\perp}} \mathrm{d} \mathbf{k}_{\perp}\left[\mathrm{e}^{i \mathbf{Q} \cdot \mathbf{X} / \hbar} L\left(\mathbf{k}_{\perp}, \mathrm{P} ; \mathbf{Q}\right) \rho L^{\dagger}\left(\mathbf{k}_{\perp}, \mathrm{P} ; \mathbf{Q}\right) \mathrm{e}^{-i \mathbf{Q} \cdot \mathbf{X} / \hbar}\right. \\
& \left.-\frac{1}{2}\left\{\rho, L^{\dagger}\left(\mathbf{k}_{\perp}, \mathrm{P} ; \mathbf{Q}\right) L\left(\mathbf{k}_{\perp}, \mathrm{P} ; \mathbf{Q}\right)\right\}\right]
\end{aligned}
$$

where $\mathrm{X}$ and $\mathrm{P}$ are the position and the momentum operator of the test particle, and the curly brackets denote the anti-commutator. The integration is over all momentum transfers $\mathbf{Q}$, and over gas particle momenta $\mathbf{p}$ from the plane perpendicular to $\mathbf{Q}$, i.e., from $\mathbf{Q}^{\perp} \equiv\left\{\mathbf{p} \in \mathbb{R}^{3}: \mathbf{p} \cdot \mathbf{Q}=0\right\}$. The function $L$, which appears operator-valued in (2.2), contains all the details of the collisional interaction with the gas. It is defined by

$$
\begin{aligned}
L(\mathbf{p}, \mathbf{P} ; \mathbf{Q})= & \left.\sqrt{\frac{n_{\text {gas }} m}{m_{*}^{2} Q} f\left(\operatorname{rel}\left(\mathbf{p}_{\perp \mathbf{Q}}, \mathbf{P}_{\perp \mathbf{Q}}\right)-\frac{\mathbf{Q}}{2}\right.}, \operatorname{rel}\left(\mathbf{p}_{\perp \mathbf{Q}}, \mathbf{P}_{\perp \mathbf{Q}}\right)+\frac{\mathbf{Q}}{2}\right) \\
& \times \sqrt{\mu\left(\mathbf{p}_{\perp \mathbf{Q}}+\frac{m}{m_{*}} \frac{\mathbf{Q}}{2}+\frac{m}{M} \mathbf{P}_{\| \mathbf{Q}}\right)},
\end{aligned}
$$

where $m$ and $M$ are the masses of the gas particles and of the test particle, respectively, and $m_{*}=$ $m M /(m+M)$ denotes the reduced mass. It involves also the gas number density $n_{\text {gas }}$, the distribution function $\mu(\mathbf{p})$ of the gas momenta, and, most importantly, the elastic scattering amplitude $f\left(\mathbf{p}_{f}, \mathbf{p}_{i}\right)$ determined by the two-body interaction between gas and test particle. The subscripts $\| \mathbf{Q}$ and $\perp \mathbf{Q}$ denote the component of a vector parallel and perpendicular to the momentum transfer $\mathbf{Q}$, such that $\mathbf{P} \| \mathbf{Q}=(\mathbf{P} \cdot \mathbf{Q}) \mathbf{Q} / Q^{2}$ and $\mathbf{P}_{\perp \mathbf{Q}}=\mathbf{P}-\mathbf{P}_{\| \mathbf{Q}}$ respectively. Finally, relative momenta are stated by means of the abbreviation

$$
\operatorname{rel}(\mathbf{p}, \mathbf{P}) \equiv \frac{m_{*}}{m} \mathbf{p}-\frac{m_{*}}{M} \mathbf{P} \text {. }
$$

The Hamiltonian correction in (2.1) is defined by the function 32]

$$
H_{\mathrm{n}}(\mathbf{P})=-2 \pi \hbar^{2} \frac{n_{\text {gas }}}{m_{*}} \int \mathrm{d} \mathbf{p} \mu(\mathbf{p}) \operatorname{Re}[f(\operatorname{rel}(\mathbf{p}, \mathbf{P}), \operatorname{rel}(\mathbf{p}, \mathbf{P}))] .
$$

As discussed in Sect. 6, this energy shift is related to forward scattering.

The quantum linear Boltzmann equation is valid for non-degenerate, uniform gases with arbitrary stationary momentum distribution $\mu(\mathbf{p})$. However, for concrete calculations we will often tacitly take $\mu(\mathbf{p})$ to be the standard Maxwell-Boltzmann expression

$$
\mu_{\beta}(\mathbf{p})=\frac{1}{\pi^{3 / 2} p_{\beta}^{3}} \exp \left(-\frac{\mathbf{p}^{2}}{p_{\beta}^{2}}\right)
$$

with $p_{\beta}=\sqrt{2 m / \beta}$ the most probable momentum at temperature $T=1 /\left(k_{\mathrm{B}} \beta\right)$. 


\subsection{Momentum representation}

To gain some physical insight into the equation it is convenient to express it in the basis of improper momentum eigenstates, $\mathbf{P}|\mathbf{P}\rangle=\mathbf{P}|\mathbf{P}\rangle$, which will also help in establishing the relation to its classical counterpart. In the momentum representation Eq. (2.2) can be brought into the form

$$
\left\langle\mathbf{P}|\mathcal{L} \rho| \mathbf{P}^{\prime}\right\rangle=\int \mathrm{d} \mathbf{Q} M_{\text {in }}\left(\mathbf{P}, \mathbf{P}^{\prime} ; \mathbf{Q}\right)\left\langle\mathbf{P}-\mathbf{Q}|\rho| \mathbf{P}^{\prime}-\mathbf{Q}\right\rangle-\frac{1}{2}\left[M_{\text {out }}^{\text {cl }}(\mathbf{P})+M_{\text {out }}^{\text {cl }}\left(\mathbf{P}^{\prime}\right)\right]\left\langle\mathbf{P}|\rho| \mathbf{P}^{\prime}\right\rangle .
$$

The "gain term", i.e., the first term on the right-hand side, is here given by

$$
M_{\text {in }}\left(\mathbf{P}, \mathbf{P}^{\prime} ; \mathbf{Q}\right)=\int_{\mathbf{Q}^{\perp}} \mathrm{d} \mathbf{k}_{\perp} L\left(\mathbf{k}_{\perp}, \mathbf{P}-\mathbf{Q} ; \mathbf{Q}\right) L^{*}\left(\mathbf{k}_{\perp}, \mathbf{P}^{\prime}-\mathbf{Q} ; \mathbf{Q}\right)
$$

with $L$ defined in Eq. (2.3). Importantly, 2.8) reduces to the rate known from the classical linear Boltzmann equation if one approaches the diagonals $\mathbf{P}=\mathbf{P}^{\prime}$,

$$
M_{\text {in }}(\mathbf{P}, \mathbf{P} ; \mathbf{Q})=M_{\text {in }}^{\mathrm{cl}}(\mathbf{P} ; \mathbf{Q}) .
$$

This classical gain rate, which involves the quantum mechanically defined differential cross-section $\sigma\left(\mathbf{p}_{f}, \mathbf{p}_{i}\right)=$ $\left|f\left(\mathbf{p}_{f}, \mathbf{p}_{i}\right)\right|^{2}$, reads as

$$
\begin{aligned}
M_{\mathrm{in}}^{\mathrm{cl}}(\mathbf{P}+\mathbf{Q} ; \mathbf{Q})= & \frac{n_{\mathrm{gas}} m}{m_{*}^{2} Q} \int_{\mathbf{Q}^{\perp}} \mathrm{d} \mathbf{k}_{\perp} \mu\left(\mathbf{k}_{\perp}+\frac{m}{m_{*}} \frac{\mathbf{Q}}{2}+\frac{m}{M} \mathbf{P}_{\| \mathbf{Q}}\right) \\
& \times \sigma\left(\operatorname{rel}\left(\mathbf{k}_{\perp}, \mathbf{P}_{\perp \mathbf{Q}}\right)-\frac{\mathbf{Q}}{2}, \operatorname{rel}\left(\mathbf{k}_{\perp}, \mathbf{P}_{\perp \mathbf{Q}}\right)+\frac{\mathbf{Q}}{2}\right) .
\end{aligned}
$$

It has a natural meaning in the sense that the transition rate

$$
M^{\mathrm{cl}}\left(\mathbf{P}_{i} \rightarrow \mathbf{P}_{f}\right) \equiv M_{\mathrm{in}}^{\mathrm{cl}}\left(\mathbf{P}_{f} ; \mathbf{P}_{f}-\mathbf{P}_{i}\right)
$$

provides the rate of collisions which change the test particle momentum from $\mathbf{P}_{i}$ to $\mathbf{P}_{f}$, or equivalently, which lead to a final momentum $\mathbf{P}_{f}$ due to a gain of momentum $\mathbf{Q}=\mathbf{P}_{f}-\mathbf{P}_{i}$.

The loss term in (2.7) is given by the arithmetic mean of the loss rates which appear in the corresponding classical equation. The latter are related to the classical gain rate by

$$
M_{\mathrm{out}}^{\mathrm{cl}}(\mathbf{P})=\int \mathrm{d} \mathbf{Q} M_{\mathrm{in}}^{\mathrm{cl}}(\mathbf{P}+\mathbf{Q} ; \mathbf{Q})
$$

This relationship, which ensures that the probability is conserved in the classical case, follows directly from the structure of (2.2), and it guarantees the normalization of the statistical operator $\rho$.

If fact, by considering Eq. (2.7) only on the diagonal,

$$
\langle\mathbf{P}|\mathcal{L} \rho| \mathbf{P}\rangle=\int \mathrm{d} \mathbf{Q} M_{\text {in }}^{\mathrm{cl}}(\mathbf{P} ; \mathbf{Q})\langle\mathbf{P}-\mathbf{Q}|\rho| \mathbf{P}-\mathbf{Q}\rangle-M_{\text {out }}^{\mathrm{cl}}(\mathbf{P})\langle\mathbf{P}|\rho| \mathbf{P}\rangle
$$

one immediately recovers one of the possible expressions of the classical linear Boltzmann equation, as we shall see in Sect. 3. It takes the explicit form

$$
\begin{aligned}
\langle\mathbf{P}|\mathcal{L} \rho| \mathbf{P}\rangle= & \frac{n_{\text {gas }} m}{m_{*}^{2}} \int \frac{\mathrm{d} \mathbf{Q}}{Q} \int_{\mathbf{Q}^{\perp}} \mathrm{d} \mathbf{k}_{\perp} \sigma\left(\operatorname{rel}\left(\mathbf{k}_{\perp}, \mathbf{P}_{\perp \mathbf{Q}}\right)-\frac{\mathbf{Q}}{2}, \operatorname{rel}\left(\mathbf{k}_{\perp}, \mathbf{P}_{\perp \mathbf{Q}}\right)+\frac{\mathbf{Q}}{2}\right) \\
& \times\left[\mu\left(\mathbf{k}_{\perp}+\frac{m}{m_{*}} \frac{\mathbf{Q}}{2}+\frac{m}{M}\left(\mathbf{P}_{\| \mathbf{Q}}-\mathbf{Q}\right)\right)\langle\mathbf{P}-\mathbf{Q}|\rho| \mathbf{P}-\mathbf{Q}\rangle\right. \\
& \left.-\mu\left(\mathbf{k}_{\perp}+\frac{m}{m_{*}} \frac{\mathbf{Q}}{2}+\frac{m}{M} \mathbf{P}_{\| \mathbf{Q}}\right)\langle\mathbf{P}|\rho| \mathbf{P}\rangle\right] .
\end{aligned}
$$




\subsection{Born approximation}

The weak coupling limit of the quantum linear Boltzmann equation is obtained if one replaces the scattering amplitudes in Eq. 2.3) by their Born approximation. This simplifies the equation considerably, since the Born amplitude associated to the interaction potential $V(\mathbf{x})$ depends only on the difference of the momenta according to

$$
\begin{aligned}
f_{B}\left(\mathbf{p}_{f}, \mathbf{p}_{i}\right) & =-4 \pi^{2} \hbar m_{*}\left\langle\mathbf{p}_{f}|V(\mathbf{x})| \mathbf{p}_{i}\right\rangle \\
& =-\frac{m_{*}}{2 \pi \hbar^{2}} \int \mathrm{d} \mathbf{x} V(\mathbf{x}) \exp \left(-i \frac{\left(\mathbf{p}_{f}-\mathbf{p}_{i}\right) \cdot \mathbf{x}}{\hbar}\right) \\
& \equiv f_{B}\left(\mathbf{p}_{f}-\mathbf{p}_{i}\right) .
\end{aligned}
$$

This removes the operator-valuedness of the scattering amplitudes in (2.2) because the function (2.3) reduces to the form

$$
L(\mathbf{p}, \mathbf{P} ; \mathbf{Q}) \rightarrow \sqrt{\frac{n_{\mathrm{gas}} m}{m_{*}^{2} Q}} f_{B}(-\mathbf{Q}) \sqrt{\mu\left(\mathbf{p}_{\perp \mathbf{Q}}+\frac{m}{m_{*}} \frac{\mathbf{Q}}{2}+\frac{m}{M} \mathbf{P}_{\| \mathbf{Q}}\right)},
$$

such that the $\mathrm{d} \mathbf{k}_{\perp}$-integration in Eq. (2.2) can be carried out if one assumes the Maxwell-Boltzmann distribution (2.6) . The resulting equation then reduces to the one proposed in 42 . Introducing the operators

$$
L_{B}(\mathrm{P} ; \mathbf{Q})=\sqrt{\sqrt{\frac{\beta m}{2 \pi}} \frac{n_{\mathrm{gas}} \sigma_{B}(\mathbf{Q})}{m_{*}^{2} Q}} \exp \left(-\beta \frac{\left(\left(1+\frac{m}{M}\right) Q^{2}+2 \frac{m}{M} \mathrm{P} \cdot \mathbf{Q}\right)^{2}}{16 m Q^{2}}\right),
$$

with $\sigma_{B}=\left|f_{B}\right|^{2}$, it takes the simpler form

$$
\mathcal{L}_{B} \rho=\int \mathrm{d} \mathbf{Q}\left[\mathrm{e}^{i \mathbf{Q} \cdot \mathbf{X} / \hbar} L_{B}(\mathrm{P} ; \mathbf{Q}) \rho L_{B}^{\dagger}(\mathrm{P} ; \mathbf{Q}) \mathrm{e}^{-i \mathbf{Q} \cdot \mathbf{X} / \hbar}-\frac{1}{2}\left\{\rho, L_{B}^{\dagger}(\mathrm{P} ; \mathbf{Q}) L_{B}(\mathrm{P} ; \mathbf{Q})\right\}\right]
$$

where only the integration over the momentum transfers is left.

We note that Eq. (2.2) can be simplified also by other limiting procedures. The minimally extended Caldeira-Leggett master equation is obtained in the diffusive limit described in Sect. 5.2, while the master equation of pure collisional decoherence discussed in Sect. 7.1 is gained in the limit of a very massive tracer particle, $m / M \rightarrow 0$.

\subsection{Survey and comparison of derivations in the literature}

The quantum linear Boltzmann equation has a long history, even though the general form Eq. (2.2) was obtained only recently. The key difficulty lies in the necessity to account for the collisional dynamics in a microscopically realistic and non-perturbative way, leading to a dynamics which is consistent with the semigroup structure of a proper Markovian description and which displays the thermalization behavior known from the classical description.

A Boltzmann-like master equation already appeared in the seminal work by Joos and Zeh [40] which used a scattering theory description to treat the decoherence effect of environmental collisions on the motion of a quantum particle. Despite its similar name the master equation presented there is quite different from Eq. (2.2) in that it linearizes the interaction effect and neglects dissipation, thus leading to an infinite growth of the kinetic energy of the test particle on long time scales. Indeed, the equation is meant to describe only the short-time loss of coherence in the motional state. However, due to the linearization involved the predicted localization rate grows above all bounds for increasingly delocalized quantum states, which may quickly lead to unrealistically large decoherence rates.

This result was improved by Gallis and Fleming [43, who proposed a master equation using the full scattering matrix. This equation predicts a saturation of the localization rate at large distances, as one 
expects for local interactions with the environment, though at a numerically incorrect value. Like the master equation by Joos and Zeh, this result does not account for dissipation phenomena.

To our knowledge, the first proposal pointing to a quantum linear Boltzmann equation which also describes energy relaxation and therefore grants the existence of a stationary solution, was given by Diòsi [4]. As discussed in Sect. 3.4, this result is not equivalent to what we consider the correct quantum linear Boltzmann equation, although the diagonal matrix elements in the momentum representation coincide, yielding the expected classical equation.

The correct result including both decoherence and dissipative effects, though restricted to a perturbative scattering cross-section evaluated in Born approximation, was later obtained in [45|46|42]47]. This work also pointed out an important connection between the collision kernel in the quantum linear Boltzmann equation and a two-point correlation function of the medium known as dynamic structure factor, first introduced by van Hove for the description of scattering of a test particle off a macroscopic sample. In the master equation the dynamic structure factor, given by the Fourier transform of the time dependent density autocorrelation function of the gas, appears operator-valued and accounts for the existence of the canonical stationary solution, as discussed in detail in Sect. 8. At the same time, the connection between this quantum master equation and the general structure of translation-covariant master equations obtained by Holevo [33] was first explored and clarified.

The final, non-perturbative version of the quantum linear Boltzmann equation, which is discussed in the present article, was first derived in [41. It is noteworthy that this result is not obtained from the weak coupling expression by naively replacing the Born approximation of the scattering amplitude with the exact expression, since this procedure is not unambiguous. Indeed, the substitution of $\sigma_{B}^{1 / 2}$ in (2.17) by the exact scattering amplitude would yield an expression very different from the correct equation as given by (2.2) and (2.3), which requires an additional two-dimensional integration.

Other recent attempts to derive master equations related to the effect of a gas environment are due to Alicki 48 and Dodd and Halliwell 49] (see also 50|51/52/53 for recent mathematical work building on the covariance under translations). These papers focus on the collisional decoherence effect for the case of a very massive particle, where dissipative effects are not accounted for. In this regard, a reexamination of the derivation of the master equation by Gallis and Fleming was presented in [54. That article discusses several ways how to consistently incorporate scattering theory into a Markovian description, which served to ascertain the numerical value of the localization rate due to collisional decoherence, thus confirming the prefactor implied by [4446]. The importance of an accurate description of collisional decoherence was demonstrated by experimental measurements with interfering fullerene molecules [3955, which were sensitive to the exact saturation value of the localization rate. We note that the techniques used in 554 were also instrumental for developing the methods required to combine asymptotic scattering theory with a dynamic description of the state evolution, as required in the derivation of the final form of the quantum linear Boltzmann equation.

Retrospectively, one can distinguish two rather different approaches followed in the various attempts at deriving the master equation discussed in the present article. One possible line of argumentation starts from the microscopic Hamiltonian for the joint system of test particle and gas, with an interaction term specified by a potential depending on the relative distance between gas particle and test particle. A natural mathematical framework in this approach is the formalism of second quantization. In this non-relativistic quantum field theoretical setting the interaction is naturally expressed as a density-density coupling. In this context a second order perturbation expansion leads to the Fourier transform of the density autocorrelation function of the gas, that is to its dynamic structure factor [56. The result includes statistical corrections, which are naturally accounted for in a field theoretical formalism. However, the restriction to the second order term involves the Fourier transform of the interaction potential, corresponding to the Born approximation for the scattering cross-section. Going beyond the second order term, thus recovering the full T-matrix including statistical corrections, becomes a very difficult task, which has still not been fully accomplished. This standpoint has been considered in [57/58/42/49].

A second line of reasoning starts from the description of single collision events, formulated by means of scattering theory, which has also recently been the object of mathematical investigations [59]60]61]62]63]. While this approach admits a non-perturbative description of the effect of a collision, it cannot easily account 
for degeneracy effects, which may occur if the gas particles are to be considered as being indistinguishable. The dynamic effect of the gas is essentially described by weighting the effect of a single collision, obtained by tracing over the scattered gas particle, with the rate of collisions. This reasoning was pursued in a phenomenological sense in 6444]. However, since both the rate of collisions and the effect of a single collision depend in general on the particle state, it is not straightforward to derive an expression for the infinitesimal state evolution which is linear in the density operator, as required in a Markovian master equation. It is achieved in a line of reasoning termed the monitoring approach [65[66], which is based on concepts drawn from the theory of generalized and continuous measurements [286768]. It introduces a positive operator for the scattering rate and thus yields an expression for the dynamic state evolution, involving the S-matrix and square roots of the rate operator, which is manifestly of Lindblad form once the environmental trace is done. This approach has been shown to yield nontrivial master equations that have been established by other means 6569. If applied to the case of a tracer particle in a gas it leads in a stringent calculation to the quantum linear Boltzmann equation, as discussed in some detail in 32 .

Finally, we note that a large number of works can be found in the literature which are related to the present subject. While it is impossible to do justice to all authors, we mention a few recent articles related to the derivation of a quantum kinetic equation in the spirit of the Boltzmann equation, both linear

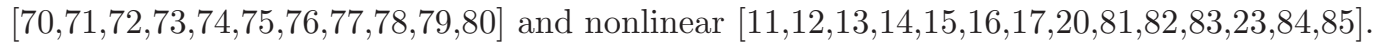

\section{A heuristic derivation}

We now present a heuristic motivation for the particular form of the quantum linear Boltzmann equation as given by Eqs. (2.2) and (2.3). It rests upon two basic requirements, its compatibility with (a) the classical linear Boltzmann equation, and with (b) the structure of the general form of a covariant completely positive master equation. Although our line of reasoning can hardly be called a proper derivation, it is rather straightforward and provides an intuitive reasoning why the particular form given above is necessary.

\subsection{The classical linear Boltzmann equation}

It is helpful to first discuss the classical version of the linear Boltzmann equation. In particular, we will rewrite it in a form that exhibits a natural correspondence with the quantum version. As already explained in Sect. 1] the classical linear Boltzmann equation describes the collisional dynamics of a test particle interacting with a homogeneous, ideal background gas characterized by the momentum distribution $\mu$ (p). The nontrivial part of the equation is therefore the collisional contribution to the time evolution of the distribution function $f(\mathbf{P})$, which we shall denote by $\partial_{t}^{\text {coll }} f(\mathbf{P})$. We start with the form

$$
\begin{aligned}
\partial_{t}^{\text {coll }} f(\mathbf{P})= & \frac{n_{\text {gas }}}{m_{*}^{2}} \int \mathrm{d} \mathbf{P}^{\prime} \int \mathrm{d}^{\prime} \int \mathrm{d} \mathbf{p} \sigma\left(\operatorname{rel}(\mathbf{p}, \mathbf{P}), \operatorname{rel}\left(\mathbf{p}^{\prime}, \mathbf{P}^{\prime}\right)\right) \\
& \times \delta\left(\frac{P^{\prime 2}}{2 M}+\frac{p^{\prime 2}}{2 m}-\frac{P^{2}}{2 M}-\frac{p^{2}}{2 m}\right) \delta^{3}\left(\mathbf{P}^{\prime}+\mathbf{p}^{\prime}-\mathbf{P}-\mathbf{p}\right)\left[\mu\left(\mathbf{p}^{\prime}\right) f\left(\mathbf{P}^{\prime}\right)-\mu(\mathbf{p}) f(\mathbf{P})\right],
\end{aligned}
$$

where the fundamental role of energy and momentum conservation in each single collision is put in the foreground. The differential scattering cross-section $\sigma$, which is a function of the relative momenta before and after a collision with a gas particle, is taken to satisfy $\sigma\left(\mathbf{p}_{f}, \mathbf{p}_{i}\right)=\sigma\left(\mathbf{p}_{i}, \mathbf{p}_{f}\right)$, reflecting the invariance of the collisional interaction under an inversion of time and parity. We note that the collision integral of the original, nonlinear Boltzmann is obtained from (3.1) by replacing $\mu$ (p) with $f(\mathbf{p})$, and by setting $M=m$.

As shown in Appendix A.1 by explicitly evaluating the two delta functions in (3.1) one arrives at the expression

$$
\begin{aligned}
\partial_{t}^{\text {coll }} f(\mathbf{P})= & \frac{n_{\text {gas }} m}{m_{*}^{2}} \int \frac{\mathrm{d} \mathbf{Q}}{Q} \int_{\mathbf{Q}^{\perp}} \mathrm{d} \mathbf{k}_{\perp} \sigma\left(\operatorname{rel}\left(\mathbf{k}_{\perp}, \mathbf{P}_{\perp \mathbf{Q}}\right)-\frac{\mathbf{Q}}{2}, \operatorname{rel}\left(\mathbf{k}_{\perp}, \mathbf{P}_{\perp \mathbf{Q}}\right)+\frac{\mathbf{Q}}{2}\right) \\
& \times\left[\mu\left(\mathbf{k}_{\perp}+\frac{m}{m_{*}} \frac{\mathbf{Q}}{2}+\frac{m}{M}\left(\mathbf{P}_{\| \mathbf{Q}}-\mathbf{Q}\right)\right) f(\mathbf{P}-\mathbf{Q})-\mu\left(\mathbf{k}_{\perp}+\frac{m}{m_{*}} \frac{\mathbf{Q}}{2}+\frac{m}{M} \mathbf{P}_{\| \mathbf{Q}}\right) f(\mathbf{P})\right] .
\end{aligned}
$$


It has already the same form as the right-hand side of Eq. (2.14). By exploiting the notation used in Eq. (2.9) and Eq. (2.12) one can write (3.2) more compactly as

$$
\partial_{t}^{\text {coll }} f(\mathbf{P})=\int \mathrm{d} \mathbf{Q} M_{\text {in }}^{\text {cl }}(\mathbf{P} ; \mathbf{Q}) f(\mathbf{P}-\mathbf{Q})-M_{\text {out }}^{\mathrm{cl}}(\mathbf{P}) f(\mathbf{P}),
$$

that is, one arrives at Eq. 2.13) upon identifying the classical momentum distribution function $f(\mathbf{P})$ with $\langle\mathbf{P}|\rho| \mathbf{P}\rangle$, the diagonal matrix elements of the statistical operator in the momentum representation. Equation (3.3) makes it immediately apparent that the classical linear Boltzmann equation takes exactly the form of a classical Markovian master equation.

\subsection{General form of a translation-covariant quantum master equation}

The classical result Eq. (3.2) already provides a hint on the quantum version of the linear Boltzmann equation, in that it tells how the diagonal matrix elements in the momentum representation must look like. However, this constraint does obviously not suffice to fix the quantum master equation, which is required to describe not only the populations in a given basis, but also the coherences corresponding to the off-diagonal elements.

As is well-known from the theory of open quantum systems [29], the general structure of a Markovian master equation is fixed by the Lindblad form, according to which the non-Hamiltonian part must have the structure

$$
\mathcal{L} \rho=\sum_{j}\left[\mathrm{~L}_{j} \rho \mathrm{L}_{j}^{\dagger}-\frac{1}{2}\left\{\mathrm{~L}_{j}^{\dagger} \mathrm{L}_{j}, \rho\right\}\right] .
$$

In the present case, however, a more powerful mathematical characterization of the master equation is available, which builds on the translational covariance of $\mathcal{L} \rho$, and thus provides further cues on the possible expressions of the Lindblad operators $\mathrm{L}_{j}$. Early results on the subject go back to one of the pioneering works on quantum dynamical semigroups [86, and the problem was later reconsidered in 87/88. The final characterization was obtained by Holevo in a series of papers 89|9019192, by building on a quantum generalization of the classical Lévy-Khintchine formula.

The linear Boltzmann equation must be invariant under translations, both in the classical and in the quantum version, because the background gas is taken to be homogeneous, while the interactions are described by a two-body potential depending on the relative distance between colliding particles. This property is immediately apparent in the structure of the classical equation. As discussed in more detail in Sect. 4.1.1. it implies on the quantum level that the master equation must be translation-covariant, that is, its action must commute with the unitary representation of translations,

$$
\mathcal{L}\left(\mathrm{e}^{-i \mathbf{A} \cdot \mathrm{P} / \hbar} \rho \mathrm{e}^{i \mathbf{A} \cdot \mathrm{P} / \hbar}\right)=\mathrm{e}^{-i \mathbf{A} \cdot \mathrm{P} / \hbar} \mathcal{L} \rho \mathrm{e}^{i \mathbf{A} \cdot \mathrm{P} / \hbar} .
$$

It was shown by Holevo in the aforementioned work that the structure of a master equation which complies with Eq. (3.5) is given by

$$
\mathcal{L} \rho=\int \mathrm{d} \mathbf{Q} \sum_{j}\left[\mathrm{e}^{i \mathbf{Q} \cdot \mathbf{X} / \hbar} L_{j}(\mathrm{P} ; \mathbf{Q}) \rho L_{j}^{\dagger}(\mathrm{P} ; \mathbf{Q}) \mathrm{e}^{-i \mathbf{Q} \cdot \mathbf{X} / \hbar}-\frac{1}{2}\left\{L_{j}^{\dagger}(\mathrm{P} ; \mathbf{Q}) L_{j}(\mathrm{P} ; \mathbf{Q}), \rho\right\}\right],
$$

with arbitrary functions $L_{j}(\mathbf{P} ; \mathbf{Q})$, while an additional Hamiltonian contribution may depend only on P.

This assumes that the mapping $\mathcal{L}$ is bounded and that the underlying statistical process is Poissonian. A more general expression including both a Gaussian and a Poisson component has to be considered if one also allows for unbounded mappings 47/5693. An example of such a Gaussian component is given by the master equation Eq. (5.10), obtained from the quantum linear Boltzmann equation in the Brownian motion limit considered in Sect. 5 ,

Although the form of Eq. (3.6) complies with the Lindblad result, it provides much richer information on the structure of the Lindblad operators. For instance, it states that the only possible dependence on 
the position operator $\mathbf{X}$ can be in the unitary momentum boost operator $\exp (i \mathbf{Q} \cdot \mathbf{X} / \hbar)$. In particular, the diagonal matrix elements of Eq. (3.6) in the momentum representation are given by

$$
\langle\mathbf{P}|\mathcal{L} \rho| \mathbf{P}\rangle=\int \mathrm{d} \mathbf{Q} \sum_{j}\left[\left|L_{j}(\mathbf{Q}, \mathbf{P}-\mathbf{Q})\right|^{2}\langle\mathbf{P}-\mathbf{Q}|\rho| \mathbf{P}-\mathbf{Q}\rangle-\left|L_{j}(\mathbf{Q}, \mathbf{P})\right|^{2}\langle\mathbf{P}|\rho| \mathbf{P}\rangle\right] .
$$

\subsection{Combining translational invariance with the classical equation}

One can now ask whether a natural choice of the Lindblad operators for the quantum linear Boltzmann equation is suggested already by combining the requirements posed by its classical analog and by the characterization of translation-covariant Markovian dynamics. Specifically, by comparing the diagonal momentum matrix elements of the covariant master equation (3.7) with the classical expression Eq. (3.3) one finds that the gain term $\sum_{j}\left|L_{j}(\mathbf{Q}, \mathbf{P}-\mathbf{Q})\right|^{2}$ in Eq. (3.7) corresponds to that expression in Eq. (3.2) which yields the classical rate $M_{\text {in }}^{\text {cl }}(\mathbf{P} ; \mathbf{Q})$ in Eq. (3.3).

It follows that the sum over the discrete set of Lindblad operators $L_{j}(\mathrm{P} ; \mathbf{Q})$ must be replaced by an integration over a two-dimensional continuous index, in order to recover the five-fold integration in Eq. (3.2). Apart from the integral over the momentum transfer $\mathbf{Q}$, this brings about an additional integration over the plane of momenta perpendicular to $\mathbf{Q}$,

$$
\sum_{j} L_{j}(\mathrm{P} ; \mathbf{Q}) \rho L_{j}^{\dagger}(\mathrm{P} ; \mathbf{Q}) \rightarrow \int_{\mathbf{Q}^{\perp}} \mathrm{d} \mathbf{k}_{\perp} L\left(\mathbf{k}_{\perp}, \mathrm{P} ; \mathbf{Q}\right) \rho L^{\dagger}\left(\mathbf{k}_{\perp}, \mathrm{P} ; \mathbf{Q}\right)
$$

with $L(\mathbf{p}, \mathrm{P} ; \mathbf{Q})$ the accordingly continuous collection of Lindblad operators. It follows from Eq. (3.2) that they must satisfy

$$
\begin{aligned}
|L(\mathbf{p}, \mathbf{P} ; \mathbf{Q})|^{2}= & \frac{n_{\text {gas }} m}{m_{*}^{2} Q} \sigma\left(\operatorname{rel}\left(\mathbf{p}_{\perp \mathbf{Q}}, \mathbf{P}_{\perp \mathbf{Q}}\right)-\frac{\mathbf{Q}}{2}, \operatorname{rel}\left(\mathbf{p}_{\perp \mathbf{Q}}, \mathrm{P}_{\perp \mathbf{Q}}\right)+\frac{\mathbf{Q}}{2}\right) \\
& \times \mu\left(\mathbf{p}_{\perp \mathbf{Q}}+\frac{m}{m_{*}} \frac{\mathbf{Q}}{2}+\frac{m}{M} \mathbf{P}_{\| \mathbf{Q}}\right) .
\end{aligned}
$$

The relation $\sigma\left(\mathbf{p}_{f}, \mathbf{p}_{i}\right)=\left|f\left(\mathbf{p}_{f}, \mathbf{p}_{i}\right)\right|^{2}$ and the positivity of $\mu$ now suggest a natural choice for the Lindblad operators,

$$
\begin{aligned}
L(\mathbf{p}, \mathrm{P} ; \mathbf{Q})= & \left.\sqrt{\frac{n_{\text {gas }} m}{m_{*}^{2} Q} f\left(\operatorname{rel}\left(\mathbf{p}_{\perp \mathbf{Q}}, \mathrm{P}_{\perp \mathbf{Q}}\right)-\frac{\mathbf{Q}}{2}\right.}, \operatorname{rel}\left(\mathbf{p}_{\perp \mathbf{Q}}, \mathrm{P}_{\perp \mathbf{Q}}\right)+\frac{\mathbf{Q}}{2}\right) \\
& \times \sqrt{\mu\left(\mathbf{p}_{\perp \mathbf{Q}}+\frac{m}{m_{*}} \frac{\mathbf{Q}}{2}+\frac{m}{M} \mathbf{P}_{\| \mathbf{Q}}\right)} .
\end{aligned}
$$

This expression, which involves a complex, operator-valued scattering amplitude, was obtained here in a rather straightforward and intuitive way. It is identical with the result (2.3), derived stringently by means of quantum scattering theory within the monitoring approach.

\subsection{Inequivalent expressions}

The previous section presented a rather suggestive line of reasoning in support of the expression Eq. (2.3). We emphasize, however, that these arguments are not fully conclusive, since one can formulate quantum master equations different from Eq. (2.2), which still comply with Eq. (3.2) and Eq. (3.6). Incidentally, this is the case for a proposal by Diòsi [44, aiming at the generalization of the classical linear Boltzmann equation. Clearly, the remaining freedom lies in the fact that the diagonal matrix elements in the momentum representation do not uniquely fix the mapping $\mathcal{L}$. The quantum linear Boltzmann equation is an operator equation for the density matrix, after all, while its classical version governs only a probability distribution. 
In order to pinpoint the remaining freedom and the connection to Diòsi's result [44] let us return to the classical expression Eq. (3.2). This equation is invariant under a change of integration variables of the form

$$
\mathbf{k}_{\perp} \rightarrow a\left(\mathbf{k}_{\perp}, \mathbf{P} ; \mathbf{Q}\right) \mathbf{k}_{\perp}+b\left(\mathbf{k}_{\perp}, \mathbf{P} ; \mathbf{Q}\right) \mathbf{P}_{\perp \mathbf{Q}}
$$

with $a$ and $b$ arbitrary scalar functions. This changes the dependence on the test particle momentum $\mathbf{P}$ in the explicit expression of the classical gain term, such that different master equations are obtained when promoting $\mathbf{P}$ to an operator and reading off the Lindblad operators, as done above in Eqs. (3.9) and (3.10). These various master equations coincide with respect to the diagonal in momentum representation, because one can translate back the integration variables when evaluating those matrix elements.

In particular, by choosing $a=1$ and $b=m / M$ the classical linear Boltzmann equation takes the form

$$
\begin{aligned}
\partial_{t}^{\text {coll }} f(\mathbf{P})= & \frac{n_{\text {gas }} m}{m_{*}^{2}} \int \frac{\mathrm{d} \mathbf{Q}}{Q} \int_{\mathbf{Q}^{\perp}} \mathrm{d} \mathbf{k}_{\perp} \sigma\left(\frac{m_{*}}{m} \mathbf{k}_{\perp}-\frac{\mathbf{Q}}{2}, \frac{m_{*}}{m} \mathbf{k}_{\perp}+\frac{\mathbf{Q}}{2}\right) \\
& \times\left[\mu\left(\mathbf{k}_{\perp}+\frac{m}{M} \mathbf{P}_{\perp \mathbf{Q}}+\frac{m}{m_{*}} \frac{\mathbf{Q}}{2}+\frac{m}{M}\left(\mathbf{P}_{\| \mathbf{Q}}-\mathbf{Q}\right)\right) f(\mathbf{P}-\mathbf{Q})\right. \\
& \left.-\mu\left(\mathbf{k}_{\perp}+\frac{m}{M} \mathbf{P}_{\perp \mathbf{Q}}+\frac{m}{m_{*}} \frac{\mathbf{Q}}{2}+\frac{m}{M} \mathbf{P}_{\| \mathbf{Q}}\right) f(\mathbf{P})\right]
\end{aligned}
$$

In this case, the constraint on the Lindblad operators corresponding to Eq. (3.9) reads as

$$
\begin{aligned}
|L(\mathbf{p}, \mathbf{P} ; \mathbf{Q})|^{2}= & \frac{n_{\text {gas }} m}{m_{*}^{2} Q} \sigma\left(\frac{m_{*}}{m} \mathbf{p}_{\perp \mathbf{Q}}-\frac{\mathbf{Q}}{2}, \frac{m_{*}}{m} \mathbf{p}_{\perp \mathbf{Q}}+\frac{\mathbf{Q}}{2}\right) \\
& \times \mu\left(\mathbf{p}_{\perp \mathbf{Q}}+\frac{m}{m_{*}} \frac{\mathbf{Q}}{2}+\frac{m}{M} \mathbf{P}\right) .
\end{aligned}
$$

In the same spirit as above, this would lead to the identification

$$
\begin{aligned}
& L_{D}(\mathbf{p}, \mathbf{P} ; \mathbf{Q})=\sqrt{\frac{n_{\text {gas }} m}{m_{*}^{2} Q}} f\left(\frac{m_{*}}{m} \mathbf{p}_{\perp \mathbf{Q}}-\frac{\mathbf{Q}}{2}, \frac{m_{*}}{m} \mathbf{p}_{\perp \mathbf{Q}}+\frac{\mathbf{Q}}{2}\right) \\
& \times \sqrt{\mu\left(\mathbf{p}_{\perp \mathbf{Q}}+\frac{m}{m_{*}} \frac{\mathbf{Q}}{2}+\frac{m}{M} \mathbf{P}\right)},
\end{aligned}
$$

which corresponds to Diòsi's result 44] written in operator form. Unlike the quantum linear Boltzmann equation (2.3), the scattering amplitude is here not operator-valued, since it is independent of $P$.

Other choices of $a$ and $b$ can lead to further equations of motion. They all recover the classical linear Boltzmann equation, since their diagonal momentum matrix elements coincide, granting in particular the existence of a stationary solution. However, they differ in general as far as the description of quantum effects is concerned. For instance, the diffusive limit considered in Sect. 5 will lead to different master equations of quantum Brownian motion. In this respect, the assignment (3.10), and therefore the quantum linear Boltzmann equation, is distinguished by the fact that it leads to the minimal correction of the CaldeiraLeggett master equation [29, as discussed in Sect. 5]

As another distinguishing feature of the assignment (3.10), the complex rate $M_{\text {in }}\left(\mathbf{P}, \mathbf{P}^{\prime} ; \mathbf{Q}\right)$ resulting from Eq. (2.8) has a physically meaningful form. It is an integration over all those pairs of scattering amplitudes $f$ and $f^{*}$ which are defined by the value of $\mathbf{P}$ and of $\mathbf{P}^{\prime}$, respectively, together with the requirement of energy and momentum conservation for a given choice of $\mathbf{Q}$ and the initial gas particle momentum [66]. In an assignment like (3.14), in contrast, test particle momenta with different energies $P^{2} \neq\left(P^{\prime}\right)^{2}$ would contribute with some common scattering cross-section which does not correspond to the initial and final relative momenta determined by $\mathbf{P}, \mathbf{P}^{\prime}$, and $\mathbf{Q}$. 


\section{Properties of the quantum linear Boltzmann equation}

We now address features of the quantum linear Boltzmann equation, which are the quantum counterpart of properties also exhibited by the classical version. We start by considering the behavior under symmetry transformations. Then the relaxation dynamics predicted by the equation is addressed, and in particular the approach to an equilibrium state. As in the classical case, the latter can be understood independently of the details of scattering cross-section and gas properties. This thermalization behavior is exemplified by means of numerical results based on stochastic unravellings which exploit the particular form of the master equation.

\subsection{Symmetry transformations}

\subsubsection{Spatial translations}

A basic symmetry property of the quantum linear Boltzmann equation is its invariance under spatial translations, which was already employed in Sect. 3. One expects this to hold because the collisional interaction is due to a two-body potential which depends on the relative distance between test and gas particle. Together with the homogeneity of the gas this implies that a transformation to a frame of reference translated by a vector $\mathbf{A}$ should not affect the dynamics. This property is expressed at the mathematical level by requiring that the mapping yielding the infinitesimal time evolution commutes with the action of the unitary representation of translations, as expressed by Eq. (3.5). It implies in particular that if the statistical operator $\rho$ is a solution of the master equation, the translated operator $\mathrm{e}^{-i \mathbf{A} \cdot \mathrm{P} / \hbar} \rho \mathrm{e}^{i \mathbf{A} \cdot \mathrm{P} / \hbar}$ also provides a solution.

To check this invariance of the quantum linear Boltzmann equation let us consider the effect of a translation by a vector $\mathbf{A}$ on the superoperator $\mathcal{M}$ appearing in Eq. (2.1). We here recall its explicit expression

$$
\begin{aligned}
\mathcal{M} \rho= & -\frac{i}{\hbar}\left[\mathrm{H}_{0}+H_{\mathrm{n}}(\mathrm{P}), \rho\right]+\int \mathrm{d} \mathbf{Q} \int_{\mathbf{Q}^{\perp}} \mathrm{d} \mathbf{k}_{\perp}\left[\mathrm{e}^{i \mathbf{Q} \cdot \mathrm{X} / \hbar} L\left(\mathbf{k}_{\perp}, \mathrm{P} ; \mathbf{Q}\right) \rho L^{\dagger}\left(\mathbf{k}_{\perp}, \mathrm{P} ; \mathbf{Q}\right) \mathrm{e}^{-i \mathbf{Q} \cdot \mathrm{X} / \hbar}\right. \\
& \left.-\frac{1}{2}\left\{\rho, L^{\dagger}\left(\mathbf{k}_{\perp}, \mathrm{P} ; \mathbf{Q}\right) L\left(\mathbf{k}_{\perp}, \mathrm{P} ; \mathbf{Q}\right)\right\}\right],
\end{aligned}
$$

with $L(\mathbf{p}, \mathrm{P} ; \mathbf{Q})$ as in Eq. (2.3) and $H_{\mathrm{n}}(\mathrm{P})$ as in Eq. (2.5).

The mapping in the transformed frame of reference is then given by

$$
\mathcal{M}_{\mathbf{A}}[\rho]=\mathrm{e}^{i \mathbf{A} \cdot \mathbf{P} / \hbar} \mathcal{M}\left[\mathrm{e}^{-i \mathbf{A} \cdot \mathbf{P} / \hbar} \rho \mathrm{e}^{i \mathbf{A} \cdot \mathbf{P} / \hbar}\right] \mathrm{e}^{-i \mathbf{A} \cdot \mathbf{P} / \hbar},
$$

and we have to verify that $\mathcal{M}_{\mathbf{A}}=\mathcal{M}$.

This is easily checked by separately considering the commutator term and the incoherent part in (4.1). The Hamiltonian terms are only functions of the momentum operator $\mathrm{P}$ and therefore invariant under translations. In the incoherent part, the specific form of $L(\mathbf{p}, \mathrm{P} ; \mathbf{Q})$ is not important, since it commutes in any case with $\exp (i \mathbf{A} \cdot \mathbf{P} / \hbar)$, while the unitary operators $\exp (i \mathbf{Q} \cdot \mathbf{X} / \hbar)$ and $\exp (-i \mathbf{Q} \cdot \mathbf{X} / \hbar)$ are modified by two opposite phases which cancel out. The invariance clearly relies on the special way in which the position operator $X$ appears in Eq. (4.1).

\subsubsection{Velocity transformations}

The collisional physics underlying the quantum linear Boltzmann equation not only fixes its behavior under translations, but also under velocity transformations. This is due to the fact that the interaction rate depends on the relative velocity between the tracer particle and the gas particles, the latter being drawn from the momentum distribution of the gas particles. A transformation to a reference frame that moves with constant velocity $\mathbf{V}$ should therefore be equivalent to performing the corresponding shift in the momentum distribution of the gas particles.

Let us therefore consider the superoperator of the infinitesimal time evolution $\mathcal{M}_{\mathbf{V}}$, valid in a frame of reference which moves with velocity $\mathbf{V}$ compared to the original frame, 


$$
\mathcal{M}_{\mathbf{V}}[\rho]=\mathrm{e}^{i M \mathbf{V} \cdot \mathbf{X} / \hbar} \mathcal{M}\left[\mathrm{e}^{-i M \mathbf{V} \cdot \mathbf{X} / \hbar} \rho \mathrm{e}^{i M \mathbf{V} \cdot \mathbf{X} / \hbar}\right] \mathrm{e}^{-i M \mathbf{V} \cdot \mathbf{X} / \hbar}
$$

where $\mathcal{M}$ is given by Eq. (4.1). According to the microscopic considerations above, we expect that $\mathcal{M}_{\mathbf{V}}$ can be obtained as well by evaluating $\mathcal{M}$ with the transformed gas momentum distribution $\mu(\mathbf{p}) \rightarrow \mu^{\mathbf{V}}(\mathbf{p})=$ $\mu(\mathbf{p}-m \mathbf{V})$.

To check this symmetry, we first observe that the free kinetic term is left untouched. The explicit form (2.5) of the gas induced energy shift also immediately grants that $H_{\mathrm{n}}(\mathrm{P}-M \mathbf{V})=H_{\mathrm{n}}^{\mathbf{V}}(\mathrm{P})$, where the superscript $\mathbf{V}$ indicates quantities evaluated with the shifted momentum distribution $\mu^{\mathbf{V}}$.

Applying the same analysis to the remaining incoherent part, we note that (4.3) transforms the second term of Eq. (4.1) into

$$
\begin{aligned}
\mathcal{L}_{\mathbf{V}}[\rho]= & \int \mathrm{d} \mathbf{Q} \int_{\mathbf{Q}^{\perp}} \mathrm{d} \mathbf{k}_{\perp}\left[\mathrm{e}^{i \mathbf{Q} \cdot \mathbf{X} / \hbar} L\left(\mathbf{k}_{\perp}, \mathrm{P}-M \mathbf{V} ; \mathbf{Q}\right) \rho L^{\dagger}\left(\mathbf{k}_{\perp}, \mathrm{P}-M \mathbf{V} ; \mathbf{Q}\right) \mathrm{e}^{-i \mathbf{Q} \cdot \mathbf{X} / \hbar}\right. \\
& \left.-\frac{1}{2}\left\{\rho, L^{\dagger}\left(\mathbf{k}_{\perp}, \mathrm{P}-M \mathbf{V} ; \mathbf{Q}\right) L\left(\mathbf{k}_{\perp}, \mathrm{P}-M \mathbf{V} ; \mathbf{Q}\right)\right\}\right] .
\end{aligned}
$$

The definition of $L(\mathbf{p}, \mathbf{P} ; \mathbf{Q})$ in Eq. (2.3) implies

$$
L(\mathbf{p}, \mathbf{P}-M \mathbf{V} ; \mathbf{Q})=L^{\mathbf{V}}\left(\mathbf{p}+m \mathbf{V}_{\perp \mathbf{Q}}, \mathbf{P} ; \mathbf{Q}\right) \text {. }
$$

By inserting this into Eq. (4.4) and performing a change of integration variables $\mathbf{k}_{\perp} \rightarrow \mathbf{k}_{\perp}^{\prime}=\mathbf{k}_{\perp}-m \mathbf{V}_{\perp \mathbf{Q}}$ one

also finds for the incoherent part that the transformation (4.3) is tantamount to the replacement $\mu \rightarrow \mu$. In our notation this proves that $\mathcal{M}_{\mathbf{V}}=\mathcal{M}^{\mathbf{V}}$, that is, boosting the reference frame is equivalent to translating the gas distribution in momentum space.

\subsubsection{Rotations}

On similar grounds one can consider the behavior of the quantum linear Boltzmann equation under spatial rotations. Let us denote by $R$ the rotation around a direction $\mathbf{n}$ by the angle $\alpha$. The mapping $\mathcal{M}_{R}$ in the rotated frame is then related to the original mapping $\mathcal{M}$ by

$$
\mathcal{M}_{R}[\rho]=\mathrm{e}^{i \alpha \mathbf{n} \cdot \mathrm{J} / \hbar} \mathcal{M}\left[\mathrm{e}^{-i \alpha \mathbf{n} \cdot \mathrm{J} / \hbar} \rho \mathrm{e}^{i \alpha \mathbf{n} \cdot \mathrm{J} / \hbar}\right] \mathrm{e}^{-i \alpha \mathbf{n} \cdot \mathrm{J} / \hbar},
$$

where $\mathrm{J}$ is the angular momentum of the test particle, acting as the generator of rotations.

Usually the collisional interaction between the particles depends only on the relative orientation of incoming and outgoing momenta, $f\left(\mathbf{p}_{f}, \mathbf{p}_{i}\right)=f\left(R \mathbf{p}_{f}, R \mathbf{p}_{i}\right)$. One expects that the quantum linear Boltzmann equation is then invariant under rotations provided the gas momentum distribution is isotropic, i.e., $\mu$ (p) $=$ $\mu(R \mathbf{p})$. Indeed, the identity $\mathcal{M}_{R}=\mathcal{M}$ is then checked easily.

\subsection{Approach to equilibrium}

\subsubsection{Detailed balance and the stationary solution}

One of the most important features of both the classical and the quantum linear Boltzmann equation is the existence of a stationary solution of the canonical form, e.g. proportional to the thermal momentum distribution $\nu_{\mathrm{EQ}}(\mathbf{P})$ given by

$$
\nu_{\mathrm{EQ}}(\mathbf{P})=\frac{1}{\pi^{3 / 2} P_{\beta}^{3}} \exp \left(-\frac{\mathbf{P}^{2}}{P_{\beta}^{2}}\right),
$$

with $P_{\beta}=\sqrt{2 M / \beta}$. It is reached asymptotically for any initial state and depends only on the mass $M$ of the test particle and on the temperature $T=1 /\left(k_{\mathrm{B}} \beta\right)$ of the gas, provided the latter is described by the Maxwell-Boltzmann distribution (2.6). We now show that the existence of this solution relies also in the quantum case on the fact that the scattering rate $M_{\text {in }}^{\text {cl }}$ obeys the so-called detailed balance condition. 
Here and in the following the Hamiltonian part of the master equation does not contribute, so that we can concentrate on the mapping $\mathcal{L}$ instead of $\mathcal{M}$.

As a first step, consider the quantum linear Boltzmann equation (2.2) for a state $\nu(\mathrm{P})$, which is only a function of the momentum operator $\mathrm{P}$

$$
\mathcal{L}[\nu(\mathrm{P})]=\int \mathrm{d} \mathbf{Q}\left[M_{\mathrm{in}}^{\mathrm{cl}}(\mathrm{P} ; \mathbf{Q}) \nu(\mathrm{P}-\mathbf{Q})-M_{\mathrm{in}}^{\mathrm{cl}}(\mathrm{P}+\mathbf{Q} ; \mathbf{Q}) \nu(\mathrm{P})\right]
$$

One says that the transition rate $M^{\mathrm{cl}}\left(\mathbf{P}_{i} \rightarrow \mathbf{P}_{f}\right) \equiv M_{\mathrm{in}}^{\mathrm{cl}}\left(\mathbf{P}_{f} ; \mathbf{P}_{f}-\mathbf{P}_{i}\right)$ obeys the detailed balance condition provided

$$
M^{\mathrm{cl}}\left(\mathbf{P}_{1} \rightarrow \mathbf{P}_{2}\right) \exp \left(-\beta \frac{P_{1}^{2}}{2 M}\right)=M^{\mathrm{cl}}\left(\mathbf{P}_{2} \rightarrow \mathbf{P}_{1}\right) \exp \left(-\beta \frac{P_{2}^{2}}{2 M}\right) .
$$

For the gain rate Eq. (2.9) this implies

$$
M_{\text {in }}^{\mathrm{cl}}(\mathbf{P}+\mathbf{Q} ; \mathbf{Q})=M_{\text {in }}^{\mathrm{cl}}(\mathbf{P} ;-\mathbf{Q}) \exp (-\beta E(\mathbf{Q}, \mathbf{P})),
$$

with $E(\mathbf{Q}, \mathbf{P})$ denoting the energy transferred to the test particle in a collision where the momentum changes from $\mathbf{P}$ to $\mathbf{P}+\mathbf{Q}$,

$$
E(\mathbf{Q}, \mathbf{P})=\frac{(\mathbf{P}+\mathbf{Q})^{2}}{2 M}-\frac{P^{2}}{2 M}=\frac{Q^{2}}{2 M}+\frac{\mathbf{Q} \cdot \mathbf{P}}{M} .
$$

This quantity also relates the Maxwell-Boltzmann distribution at different momenta,

$$
\mathrm{e}^{-\beta E(\mathbf{Q}, \mathbf{P})} \nu_{\mathrm{EQ}}(\mathbf{P})=\nu_{\mathrm{EQ}}(\mathbf{P}+\mathbf{Q}) \text {. }
$$

Based on the detailed balance condition (4.9) we therefore have

$$
\mathcal{L} \nu_{\mathrm{EQ}}(\mathrm{P})=\int \mathrm{d} \mathbf{Q}\left[M_{\mathrm{in}}^{\mathrm{cl}}(\mathrm{P} ; \mathbf{Q}) \nu_{\mathrm{EQ}}(\mathrm{P}-\mathbf{Q})-M_{\mathrm{in}}^{\mathrm{cl}}(\mathrm{P} ;-\mathbf{Q}) \nu_{\mathrm{EQ}}(\mathrm{P}+\mathbf{Q})\right],
$$

which is equal to zero because the integrand is manifestly odd in $\mathbf{Q}$. If follows that $\nu_{\mathrm{EQ}}(\mathrm{P})$ is then indeed a stationary solution.

The problem is therefore reduced to checking the validity of the detailed balance condition (4.9). This can be done considering the explicit expression for the classical rate given by Eq. (2.10) and taking as the stationary distribution function $\mu$ for the gas particles the Maxwell-Boltzmann distribution (2.6). Provided the scattering cross-section obeys $\sigma\left(\mathbf{p}_{f}, \mathbf{p}_{i}\right)=\sigma\left(\mathbf{p}_{i}, \mathbf{p}_{f}\right)$ one can then directly confirm that (4.10) holds.

Finally, we emphasize that the existence of a stationary solution can be proved in a more general and direct way by writing the quantum linear Boltzmann equation in terms of the dynamic structure factor of the gas, as will be discussed in Sect. 8 .

\subsubsection{Entropy growth}

The celebrated H-theorem for the nonlinear Boltzmann equation [2] states that the function

$$
H(t)=\int \mathrm{d} \mathbf{P} f(\mathbf{P}) \log f(\mathbf{P}),
$$

satisfies $\partial_{t} H(t) \leqslant 0$. That is, the entropy $S(t)=-k_{\mathrm{B}} H(t)$ associated to the momentum distribution function $f(\mathbf{P})$ of the self-interacting gas particles does not decrease under the evolution of the nonlinear Boltzmann equation. In particular, $\partial_{t} H(t)=0$ if and only if $f(\mathbf{P})=f_{\mathrm{EQ}}(\mathbf{P})$, with $f_{\mathrm{EQ}}(\mathbf{P})$ the canonical stationary solution given by the Maxwell-Boltzmann distribution. This tells us that $f_{\mathrm{EQ}}(\mathbf{P})$ is the only stationary solution, reached for $t \rightarrow \infty$ independently of the initial condition [94.

Unlike the proper Boltzmann equation, the classical linear Boltzmann equation describes an open system, implying that the entropy associated to the distribution function $f(\mathbf{P})$ can increase or decrease in general. 
In this case it is necessary to consider the relative entropy of $f(\mathbf{P})$, defined with respect to another reference distribution function $g(\mathbf{P})$,

$$
H(f \mid g)=\int \mathrm{d} \mathbf{P} f(\mathbf{P}) \log \frac{f(\mathbf{P})}{g(\mathbf{P})} .
$$

As a measure of how effectively one can distinguish the two distribution functions, the relative entropy provides information on how close the two distribution functions are. The quantity is non-negative, and equal to zero if and only if the two distribution functions coincide. We prove in Sect. A.2 that any solution $f(\mathbf{P})$ of the classical linear Boltzmann equation satisfies

$$
\frac{\mathrm{d}}{\mathrm{d} t} H\left(f \mid f_{\mathrm{EQ}}\right) \leqslant 0,
$$

where $f_{\mathrm{EQ}}(\mathbf{P})$ is the canonical stationary solution given by the Maxwell-Boltzmann distribution. Also in this case we have $\partial_{t} H\left(f \mid f_{\mathrm{EQ}}\right)=0$ if and only if $f(\mathbf{P})=f_{\mathrm{EQ}}(\mathbf{P})$. Eq. (4.16) thus reflects the fact that independently of the initial condition any solution approaches the stationary distribution function for $t \rightarrow$ $\infty$.

The situation is similar in the case of the quantum linear Boltzmann equation. Here one considers the quantum correspondence of the relative entropy and studies its behavior under the action of completely positive quantum dynamical semigroups 959697.

One should emphasize that the following argumentation can be put on firmer ground only by confining the whole system in a region of finite volume. In this case the generator of the dynamics is given by a bounded mapping, and the stationary solution of the canonical form is indeed a trace class operator. If the test particle can move in an infinite volume, on the other hand, an operator of this form is not of trace class since $\mathrm{P}$ has a continuous spectrum. The particle is not bounded to a finite region, and it gets completely delocalized with elapsing time.

The quantum analog of the relative entropy is given by the expression

$$
S(\rho \mid w)=k_{\mathrm{B}} \operatorname{Tr} \rho \log \rho-k_{\mathrm{B}} \operatorname{Tr} \rho \log w,
$$

where $\rho$ and $w$ are two statistical operators. According to Klein's inequality this quantity is non-negative, and equal to zero if and only if the two statistical operators are equal [98|96|97.

Taking the quantum relative entropy of the solution of the master equation $\rho_{t}=\mathrm{e}^{t \mathcal{M}} \rho$ with respect to the stationary solution $\rho_{\mathrm{EQ}}$ one is led to consider the time derivative of $S\left(\rho_{t} \mid \rho_{\mathrm{EQ}}\right)$. We can now exploit the inequality

$$
S(\mathcal{U} \rho \mid \mathcal{U} w) \leqslant S(\rho \mid w),
$$

valid for any completely positive mapping $\mathcal{U}$ 989697. It provides a quantum analog of the $\mathrm{H}$-theorem for dynamical evolutions described by quantum dynamical semigroups. In particular, for $\mathcal{U}=\mathrm{e}^{t \mathcal{M}}$, with $\mathcal{M}$ the generator of a Markovian master equation, and noting that $\rho_{\mathrm{EQ}}$ is a time translation invariant state, $\mathrm{e}^{t \mathcal{M}} \rho_{\mathrm{EQ}}=\rho_{\mathrm{EQ}}$, it follows that

$$
\frac{\mathrm{d}}{\mathrm{d} t} S\left(\rho_{t} \mid \rho_{\mathrm{EQ}}\right) \leqslant 0 .
$$

Eq. (4.19) tells us that, even though the variation of the von Neumann entropy associated to the solution $\rho_{t}=\mathrm{e}^{t \mathcal{M}} \rho$ does not have a definite sign, reflecting the fact that the system is open, one still has loss of information with elapsing time because different initial states get less and less distinguishable.

In particular, the derivative of the relative entropy with respect to time can be written as

$$
\frac{\mathrm{d}}{\mathrm{d} t} S\left(\rho_{t} \mid \rho_{\mathrm{EQ}}\right)=k_{\mathrm{B}} \operatorname{Tr}\left[\mathcal{M} \rho_{t}\left(\log \rho_{t}-\log \rho_{\mathrm{EQ}}\right)\right] .
$$

It is equal to zero if and only if $\rho_{t}$ equals the stationary solution, which is unique as a consequence of the fact that only multiples of the identity commute with the Lindblad operators $\exp (i \mathbf{Q} \cdot \mathbf{X} / \hbar) L(\mathbf{p}, \mathbf{P} ; \mathbf{Q})$ 
appearing in the quantum linear Boltzmann equation (2.1) 95|99]. This result is in complete correspondence with the classical case.

Moreover, according to Eq. (4.19) any solution of the master equation gets increasingly close to the stationary distribution function. This implies in particular that any initial state gets diagonal in momentum representation for $t \rightarrow \infty$. That is, the momentum coherences described by the off-diagonal elements decay, such that the quantum linear Boltzmann equation goes over to the classical linear Boltzmann equation, which describes the remaining populations.

\subsection{Relaxation dynamics}

\subsubsection{Momentum and energy relaxation}

The previous section considered the evolution of the statistical operator, which suggested to work in the Schrödinger picture. In contrast to that, we will now use the Heisenberg picture when focussing on the dynamics of momentum and kinetic energy, that is, on the two observables most relevant for the description of a test particle in a gas.

The Heisenberg time evolution of observables requires the adjoint mapping $\mathcal{M}^{*}$, with respect to the quantum linear Boltzmann equation. It is defined through the duality relation

$$
\operatorname{Tr}(\mathbf{A} \mathcal{M} \rho)=\operatorname{Tr}\left(\rho \mathcal{M}^{*} \mathrm{~A}\right)
$$

which connects the space of trace class operators, containing the statistical operators $\rho$, with its dual, the space of bounded observables $A$. The dynamics of a Heisenberg observable $A_{t}$ is thus given by

$$
\frac{\mathrm{d}}{\mathrm{d} t} \mathrm{~A}_{t}=\frac{1}{i \hbar}\left[\mathrm{A}_{t}, \mathrm{H}_{0}+H_{n}(\mathrm{P})\right]+\mathcal{L}^{*} \mathrm{~A},
$$

with

$$
\begin{aligned}
\mathcal{L}^{*} \mathrm{~A}= & \int \mathrm{d} \mathbf{Q} \int_{\mathbf{Q}^{\perp}} \mathrm{d} \mathbf{k}_{\perp}\left[L^{\dagger}\left(\mathbf{k}_{\perp}, \mathrm{P} ; \mathbf{Q}\right) \mathrm{e}^{-i \mathbf{Q} \cdot \mathrm{X} / \hbar} \mathrm{Ae}^{i \mathbf{Q} \cdot X / \hbar} L\left(\mathbf{k}_{\perp}, \mathrm{P} ; \mathbf{Q}\right)\right. \\
& \left.-\frac{1}{2}\left\{L^{\dagger}\left(\mathbf{k}_{\perp}, \mathrm{P} ; \mathbf{Q}\right) L\left(\mathbf{k}_{\perp}, \mathrm{P} ; \mathbf{Q}\right), \mathrm{A}\right\}\right] .
\end{aligned}
$$

We will take the Heisenberg operator to coincide with the corresponding Schrödinger observable at $t=0$, i.e., $A_{0}=A$.

An important class of observables are those which are only functions of the momentum operator, $A_{t}=$ $A_{t}(\mathrm{P})$. The equation of motion in the Heisenberg picture then simplifies considerably,

$$
\frac{\mathrm{d}}{\mathrm{d} t} \mathrm{~A}_{t}=\int \mathrm{d} \mathbf{Q} \int_{\mathbf{Q}^{\perp}} \mathrm{d} \mathbf{k}_{\perp}\left|L\left(\mathbf{k}_{\perp}, \mathrm{P} ; \mathbf{Q}\right)\right|^{2}\left[\mathrm{e}^{-i \mathbf{Q} \cdot \mathbf{X} / \hbar} \mathrm{A}_{t} \mathrm{e}^{i \mathbf{Q} \cdot \mathbf{X} / \hbar}-\mathrm{A}_{t}\right] .
$$

Note that $\mathcal{L}$, and equivalently $\mathcal{L}^{*}$, is covariant under translations in the sense of Eq. (3.5), which implies that the algebra generated by the momentum operator is left invariant. Consequently, $\mathrm{A}_{0}=A_{0}(\mathrm{P})$ implies $\mathrm{A}_{t}=A_{t}(\mathrm{P})$ for $t>0$ and therefore $\left[\mathrm{A}_{t}, \mathrm{P}\right]=0$. Recalling the definition of the quantum and the classical gain rates in Eqs. (2.8) and (2.9), we find that observables given by a function of momentum obey

$$
\frac{\mathrm{d}}{\mathrm{d} t} A_{t}(\mathrm{P})=\int \mathrm{d} \mathbf{Q} M_{\mathrm{in}}^{\mathrm{cl}}(\mathrm{P}+\mathbf{Q} ; \mathbf{Q})\left[A_{t}(\mathrm{P}+\mathbf{Q})-A_{t}(\mathrm{P})\right],
$$

in strict analogy with the classical formulation.

Let us now focus on the time evolution of the expectation values of momentum and kinetic energy, $A(\mathrm{P})=\mathrm{P}$ and $A(\mathrm{P})=\mathrm{P}^{2} /(2 M)$. Using the momentum representation one can evaluate Eq. (4.25) for these quantities by working with real numbers rather than operators. In particular, for $A(\mathbf{P})=\mathbf{P}$ the quantity 
$[A(\mathbf{P}+\mathbf{Q})-A(\mathbf{P})]$ appearing at the right-hand side of Eq. (4.25) is simply the momentum transfer $\mathbf{Q}$ in the single collision. For $A(\mathbf{P})=P^{2} /(2 M)$ it is the energy transfer $E(\mathbf{Q}, \mathbf{P})$ as given by Eq. (4.11). The positivity of $M_{\text {in }}^{\text {cl }}$ thus allows one straightforwardly to confirm from Eq. (4.25) that the change of momentum is positive when the momentum transfer is positive. Likewise, a positive energy transfer increases the energy.

The dynamic equation (4.25) for the momentum and kinetic energy can be evaluated explicitly if one assumes a constant scattering cross-section $\left|f\left(\mathbf{p}_{f}, \mathbf{p}_{i}\right)\right|^{2}=\sigma_{\text {tot }} / 4 \pi$ and a free gas of Maxwell-Boltzmann

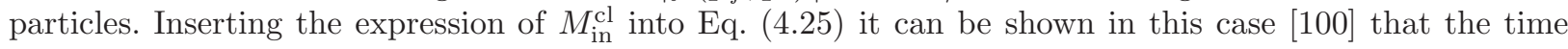
evolution of the momentum expectation value is given by

$$
\frac{\mathrm{d}}{\mathrm{d} t}\langle\mathrm{P}\rangle_{\rho_{t}}=-\frac{8}{3 \sqrt{\pi}} \frac{m_{*}}{M} \Gamma_{\beta}\left\langle\mathrm{P}_{1} F_{1}\left(-\frac{1}{2}, \frac{5}{2} ;-\left(\frac{\mathrm{P}}{M v_{\beta}}\right)^{2}\right)\right\rangle_{\rho_{t}}
$$

Accordingly, the kinetic energy $\mathrm{E}=\mathrm{P}^{2} /(2 M)$ evolves as

$$
\frac{\mathrm{d}}{\mathrm{d} t}\langle\mathrm{E}\rangle_{\rho_{t}}=-\frac{16}{3 \sqrt{\pi}} \frac{m_{*}}{M} \Gamma_{\beta}\left\langle_{1} F_{1}\left(-\frac{1}{2}, \frac{5}{2} ;-\beta \mathrm{E} \frac{m}{M}\right) \mathrm{E}-\frac{3}{2 \beta} \frac{m_{*}}{m}{ }_{1} F_{1}\left(-\frac{3}{2}, \frac{3}{2} ;-\beta \mathrm{E} \frac{m}{M}\right)\right\rangle_{\rho_{t}} .
$$

In these equations we have denoted the most probable velocity of the gas particles as $v_{\beta}=p_{\beta} / m=$ $\sqrt{2 /(\beta m)}$. Moreover,

$$
\Gamma_{\beta}=n_{\text {gas }} v_{\beta} \sigma_{\text {tot }}
$$

is the total scattering rate for a flux of particles with the most probable velocity $v_{\beta}$, and the symbol ${ }_{1} F_{1}$ denotes confluent hypergeometric functions. Their explicit expressions are given by

$$
{ }_{1} F_{1}\left(-\frac{1}{2}, \frac{5}{2} ;-x^{2}\right)=\frac{3}{16} \frac{1}{x^{2}}\left\{\left[1+2 x^{2}\right] \mathrm{e}^{-x^{2}}-\left[1-4 x^{2}-4 x^{4}\right] \frac{\sqrt{\pi}}{2} \frac{\operatorname{erf}(x)}{x}\right\}
$$

and

$$
{ }_{1} F_{1}\left(-\frac{3}{2}, \frac{3}{2} ;-x^{2}\right)=\frac{1}{8}\left\{\left[5+2 x^{2}\right] \mathrm{e}^{-x^{2}}+\left[3+12 x^{2}+4 x^{4}\right] \frac{\sqrt{\pi}}{2} \frac{\operatorname{erf}(x)}{x}\right\},
$$

which are both positive monotone increasing functions. From these expressions it is immediately clear that in general there is no closed evolution equation for either the first or the second moment of the momentum operator, $\langle\mathrm{P}\rangle_{\rho_{t}}$ or $\left\langle\mathrm{P}^{2}\right\rangle_{\rho_{t}}$, since moments of arbitrary high order are involved in the equation, due to the presence of the confluent hypergeometric functions. This becomes manifest in strong deviations of the moments from Gaussian statistics, as can be observed by studying the time dependence of high order cumulants of the momentum distribution [101.

However, the equations do get closed in the limit of a very massive test particle close to thermal equilibrium, which corresponds to the diffusive limit considered in Sect. 5. In this case, the velocity $V=P / M$ of the test particle is taken to be much smaller than the typical velocity $v_{\beta}$ of the gas particles. Thus taking $V / v_{\beta} \ll 1$ and $m / M \ll 1$ in Eq. (4.26) and Eq. (4.27) the confluent hypergeometric functions can be replaced by unity, since ${ }_{1} F_{1}(\alpha, \gamma ; 0)=1$, while the reduced mass $m_{*}$ is replaced by $m$. This leads to

$$
\frac{\mathrm{d}}{\mathrm{d} t}\langle\mathrm{P}\rangle_{\rho_{t}}=-\eta\langle\mathrm{P}\rangle_{\rho_{t}}
$$

which describes a friction proportional to velocity, leading to the expected exponential relaxation to a vanishing mean momentum. Similarly, one finds

$$
\frac{\mathrm{d}}{\mathrm{d} t}\langle\mathrm{E}\rangle_{\rho_{t}}=-2 \eta\left(\langle\mathrm{E}\rangle_{\rho_{t}}-\frac{3}{2 \beta}\right)
$$

which shows that the mean kinetic energy relaxes exponentially to the equipartition value $3 / 2 k_{\mathrm{B}} T$. The friction coefficient $\eta$ in Eq. (4.31) and (4.32) is given by 


$$
\eta=\frac{8}{3 \sqrt{\pi}} \frac{m}{M} \Gamma_{\beta}
$$

\subsubsection{Simulation algorithm}

The complexity of the quantum linear Boltzmann equation prevents its solutions from being analytically tractable, even if one allows for special choices of the scattering amplitude and the initial state. Nevertheless, one can efficiently simulate the temporal behavior of various interesting quantities by using quantum trajectory methods, which build on the Lindblad structure of the master equation and its translation-covariance. These techniques are based on stochastic differential equations for the state vector $|\psi(t)\rangle$, whose solutions are a stochastic process in the Hilbert space. A stochastic unravelling of the quantum master equation is achieved if the ensemble average $\mathbb{E}[|\psi(t)\rangle\langle\psi(t)|]$ yields a solution of the master equation [102]103[104]105|29].

One way of motivating these unravelling techniques is to consider measurement schemes, which lead to instantaneous changes of the wave vector conditioned on the random outcomes. Together with the covariant structure of the master equation described above, this consideration leads to a natural choice of the unravelling. It is a piecewise deterministic process, described by a deterministic time evolution, which gets interrupted by random jumps which change the particle momentum. This unravelling corresponds to a measurement scheme in which one monitors the momentum transferred between system and environment.

For the sake of simplicity we will restrict our analysis to the Born approximation of the equation, where the scattering cross-section depends only on the momentum transfer. Although this is not a conceptual restriction, it leads to major simplifications in the sampling of the jump events.

In Born approximation the quantum linear Boltzmann equation reads

$$
\frac{\mathrm{d}}{\mathrm{d} t} \rho=-\frac{i}{\hbar}[\mathrm{H}, \rho]+\int \mathrm{d} \mathbf{Q}\left[\mathrm{e}^{i \mathbf{Q} \cdot \mathbf{X} / \hbar} L_{B}(\mathrm{P} ; \mathbf{Q}) \rho L_{B}^{\dagger}(\mathrm{P} ; \mathbf{Q}) \mathrm{e}^{-i \mathbf{Q} \cdot \mathbf{X} / \hbar}-\frac{1}{2}\left\{L_{B}^{\dagger}(\mathrm{P} ; \mathbf{Q}) L_{B}(\mathrm{P} ; \mathbf{Q}), \rho\right\}\right]
$$

where we have used the more compact notation $\mathrm{H}=\mathrm{H}_{0}+H_{\mathrm{n}}(\mathrm{P})$ for the Hamiltonian term, and the Lindblad operators are given by Eq. (2.17).

In the Monte Carlo wave function method [105|29] one then considers a stochastic differential equation for the stochastic wave vector $\psi(t)$, which has the form

$$
\begin{aligned}
\mathrm{d}|\psi(t)\rangle= & {\left[-\frac{i}{\hbar} \mathbf{H}-\frac{\mathbf{G}}{2}+\frac{1}{2} \int \mathrm{d} \mathbf{Q} \| L_{B}(\mathbf{P} ; \mathbf{Q})|\psi(t)\rangle \|^{2}\right]|\psi(t)\rangle \mathrm{d} t } \\
& +\int \mathrm{d} \mathbf{Q}\left[\frac{\mathrm{e}^{i \mathbf{Q} \cdot \mathbf{X} / \hbar} L_{B}(\mathrm{P} ; \mathbf{Q})|\psi(t)\rangle}{\| L_{B}(\mathrm{P} ; \mathbf{Q})|\psi(t)\rangle \|}-|\psi(t)\rangle\right] \mathrm{dN}_{\mathbf{Q}}(t) .
\end{aligned}
$$

Here, the rate operator $\mathrm{G}$ is given by

$$
\mathrm{G}=\int \mathrm{d} \mathbf{Q} L_{B}^{\dagger}(\mathrm{P} ; \mathbf{Q}) L_{B}(\mathrm{P} ; \mathbf{Q}) .
$$

The field of Poisson increments $\mathrm{dN}_{\mathbf{Q}}(t)$ satisfies

$$
\begin{aligned}
\mathrm{dN}_{\mathbf{Q}}(t) \mathrm{dN}_{\mathbf{Q}^{\prime}}(t) & =\delta^{3}\left(\mathbf{Q}-\mathbf{Q}^{\prime}\right) \mathrm{dN}_{\mathbf{Q}}(t) \\
\mathbb{E}\left[\mathrm{dN}_{\mathbf{Q}}(t)\right] & =\| L_{B}(\mathbf{P} ; \mathbf{Q})|\psi(t)\rangle \|^{2} \mathrm{~d} t,
\end{aligned}
$$

where the symbol $\mathbb{E}$ denotes the ensemble mean over realizations of the process. The solutions of 4.35) starting from an initial state $|\psi(0)\rangle$ provide an unravelling of the master equation (4.34) in the sense that

$$
\rho_{t}=\mathbb{E}[|\psi(t)\rangle\langle\psi(t)|],
$$

if $\rho_{0}=|\psi(0)\rangle\langle\psi(0)|$.

In spite of the complicated form of the stochastic differential equation, which involves a field of Poisson increments, the Monte Carlo wave function method provides a rather simple simulation algorithm. The 
realizations of the process are characterized by deterministic time evolutions interrupted by jumps. If a jump into the state $|\psi(t)\rangle$ occurs at time $t$, it will evolve until the time $t+\tau$ of the next jump according to the deterministic equation

$$
|\psi(t+\tau)\rangle=\frac{\mathrm{e}^{-i \mathrm{H} \tau / \hbar-\mathrm{G} \tau / 2}|\psi(t)\rangle}{\| \mathrm{e}^{-i \mathrm{H} \tau / \hbar-\mathrm{G} \tau / 2}|\psi(t)\rangle \|} .
$$

The waiting time $\tau$ between two jumps is determined by the cumulative distribution function

$$
F(\tau)=1-\| \mathrm{e}^{-i \mathrm{H} \tau / \hbar-\mathrm{G} \tau / 2}|\psi(t)\rangle \|^{2} .
$$

A straightforward way to obtain the random variable $\tau$ numerically is to draw a random number $\eta$ from a uniform distribution over the interval $[0,1]$ and to invert the relation $\| \mathrm{e}^{-i \mathrm{H} \tau / \hbar-\mathrm{G} \tau / 2}|\psi(t)\rangle \|^{2}=\eta$. The jump is then implemented by the replacement

$$
|\psi(t+\tau)\rangle \rightarrow \frac{\mathrm{e}^{i \mathbf{Q} \cdot \mathbf{X} / \hbar} L_{B}(\mathrm{P} ; \mathbf{Q})|\psi(t+\tau)\rangle}{\| L_{B}(\mathrm{P} ; \mathbf{Q})|\psi(t+\tau)\rangle \|},
$$

where the unitary operator $\exp (i \mathbf{Q} \cdot \mathbf{X} / \hbar)$ effects a momentum kick of amount $\mathbf{Q}$. These momentum transfers must be drawn from the probability distribution

$$
\mathcal{P}(\mathbf{Q})=\frac{\| L_{B}(\mathrm{P} ; \mathbf{Q})|\psi(t)\rangle \|^{2}}{\langle\psi(t)|\mathrm{G}| \psi(t)\rangle},
$$

which is normalized by the total scattering rate out of the state $\psi(t)$,

$$
\langle\psi(t)|\mathrm{G}| \psi(t)\rangle=\int \mathrm{d} \mathbf{Q} \| L_{B}(\mathrm{P} ; \mathbf{Q})|\psi(t)\rangle \|^{2} .
$$

The random jumps in this unravelling are therefore naturally associated to the collisions with the gas molecules experienced by the test particle. The realizations of the process are thus given by a deterministic evolution interrupted by random momentum kicks corresponding to collisions. The value of the momentum transfers and the rate of collisions are fixed by the state dependent quantities Eq. (4.42) and Eq. (4.43) respectively.

The described algorithm can be implemented in a particularly simple way if the initial state is a discrete superposition of momentum eigenstates, $|\psi(0)\rangle=\sum_{j=1}^{N} c_{j}\left|\mathbf{P}_{j}\right\rangle$. Due to the translation-covariance (4.2) of the master equation superpositions of this kind are preserved during the stochastic time evolution in the sense that the quantum trajectory remains a superposition of $N$ momentum eigenstates at all times. The stochastic process is thus mapped into the space of the different amplitudes $c_{j}$ and momenta $\mathbf{P}_{j}$.

\subsubsection{The loss term}

When discussing the momentum and energy relaxation in Sect. 4.3 it was not necessary to work out the loss term of the quantum linear Boltzmann equation explicitly, since it cancels out in Eq. (4.25). However, this expression is of independent interest; it appears in the Monte Carlo simulation of the quantum linear Boltzmann equation through the rate Eq. (4.36), and it also determines the loss of visibility due to collisional decoherence in matter wave interference experiments, as we shall discuss in Sect. 7 We therefore provide an explicit expression assuming that the cross-section depends only on the relative velocity, which includes the case of a constant cross-section discussed in Sect. 4.3.

The classical loss term in the quantum linear Boltzmann equation is given by

$$
\mathcal{L}_{\text {loss }} \rho=-\frac{1}{2}\left\{M_{\text {out }}^{\mathrm{cl}}(\mathrm{P}), \rho\right\},
$$

as evident from Eq. (2.7). It is convenient to rewrite the classical loss rate $M_{\text {out }}^{\mathrm{cl}}(\mathbf{P})$, in terms of an effective scattering cross-section $\sigma_{\text {eff }}(\mathbf{P})$ defined as the area to be multiplied by the current density $n_{\text {gas }} V$ of the test particle, 


$$
M_{\text {out }}^{\mathrm{cl}}(\mathbf{P})=n_{\text {gas }} \frac{P}{M} \sigma_{\text {eff }}(\mathbf{P}) .
$$

The effective cross-section can be calculated exactly if the scattering cross-section is independent of the scattering angle (s-wave scattering), and if it depends on the energy only as a power of the relative velocity between test particle and gas particles. The microscopic cross-section can then be expressed as

$$
\sigma\left(\operatorname{rel}\left(\mathbf{p}_{\perp \mathbf{Q}}, \mathbf{P}_{\perp \mathbf{Q}}\right)-\frac{\mathbf{Q}}{2}, \operatorname{rel}\left(\mathbf{p}_{\perp \mathbf{Q}}, \mathbf{P}_{\perp \mathbf{Q}}\right)+\frac{\mathbf{Q}}{2}\right)=c\left|\frac{\mathbf{p}}{m}-\frac{\mathbf{P}}{M}\right|^{a}=c|\mathbf{v}-\mathbf{V}|^{a} .
$$

This yields the effective scattering cross-section 106

$$
\sigma_{\mathrm{eff}}(\mathbf{P})=8 \sqrt{\pi} \Gamma\left(\frac{a}{2}+2\right) \frac{M v_{\beta}^{a+1} c}{P}{ }_{1} F_{1}\left(-\left(\frac{a}{2}+\frac{1}{2}\right), \frac{3}{2} ;-\left(\frac{P}{M v_{\beta}}\right)^{2}\right),
$$

where ${ }_{1} F_{1}$ denotes the confluent hypergeometric function and $v_{\beta}=\sqrt{2 /(\beta m)}$ the most probable velocity in the gas.

For $a=0$ one recovers the case of a constant scattering cross-section $\left|f\left(\mathbf{p}_{f}, \mathbf{p}_{i}\right)\right|^{2}=\sigma_{\text {tot }} / 4 \pi$, used below to perform a Monte Carlo simulation of the quantum linear Boltzmann equation. The corresponding expression is

$$
\sigma_{\text {eff }}(\mathbf{P})=\sigma_{\text {tot }} \frac{2}{\sqrt{\pi}} \frac{M v_{\beta}}{P}{ }_{1} F_{1}\left(-\frac{1}{2}, \frac{3}{2} ;-\left(\frac{P}{M v_{\beta}}\right)^{2}\right)
$$

where

$$
{ }_{1} F_{1}\left(-\frac{1}{2}, \frac{3}{2} ;-x^{2}\right)=\left\{\frac{1}{2} \mathrm{e}^{-x^{2}}+\left[1+2 x^{2}\right] \frac{\sqrt{\pi}}{4} \frac{\operatorname{erf}(x)}{x}\right\} .
$$

For small velocities of the test particle $V / v_{\beta} \ll 1$ the expression Eq. (4.48) simplifies to

$$
\sigma_{\text {eff }}(\mathbf{P}) \stackrel{V \ll v_{\beta}}{\longrightarrow} \sigma_{\text {tot }} \frac{2}{\sqrt{\pi}} \frac{M v_{\beta}}{P}=\sigma_{\text {tot }} \frac{\bar{v}}{V},
$$

with $\bar{v}=\sqrt{8 / \pi m \beta}$ the average thermal velocity. This corresponds to the famous $1 / V$ dependence of the total scattering cross-section for small velocities, expected when the scattering probability is independent of the velocity of the tracer particle, as verified in neutron scattering 7 .

\subsubsection{Simulating the momentum and energy dynamics}

We now discuss a numerical study of the relaxation properties of the quantum linear Boltzmann equation 101, which is based on the unravelling given by Eq. 4.35) and the algorithm presented above. The use of dimensionless variables, naturally suggested by the typical physical lengths of the problem, turns out to be very convenient. Specifically, we introduce the variables

$$
\mathbf{U}=\frac{\mathbf{P}}{M v_{\beta}}=\frac{\mathbf{V}}{v_{\beta}},
$$

and

$$
\mathbf{K}=\frac{\mathbf{Q}}{m_{*} v_{\beta}} .
$$

They provide the momentum of the test particle, scaled with respect to the most probable velocity $v_{\beta}$ in the gas, as well as the scaled momentum transfer in a single collision.

For the sake of simplicity we also focus on the case of a constant cross-section. In terms of the new variables the Lindblad operators then read as 

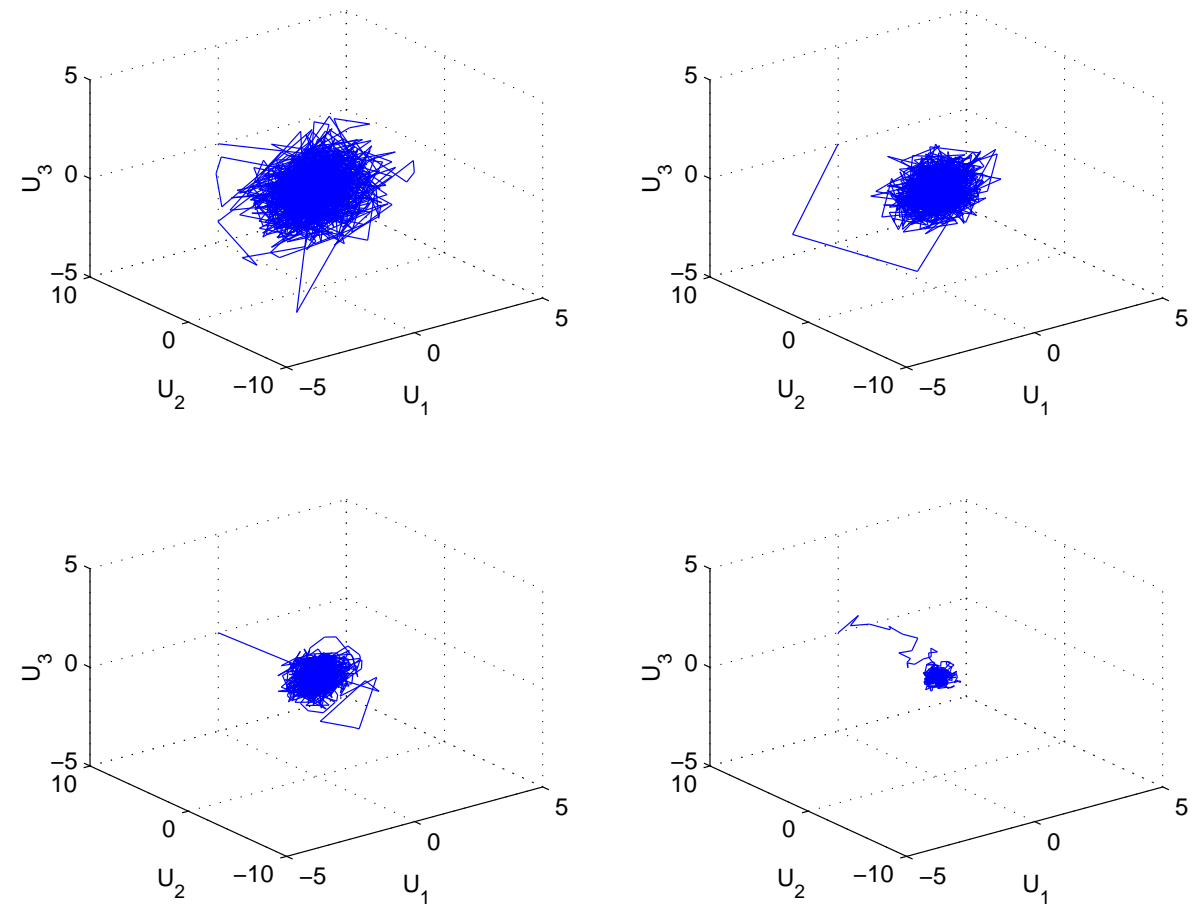

Fig. 4.1. (Color online) Single quantum trajectory realizations of the scaled momentum $\mathbf{U}(t)$, as described in Sect. 4.3 .4 The panels differ in the ratio $m / M$ between the gas and particle mass, which decreases from top left to right bottom (top row: $m / M=2$ and $m / M=1$, bottom row: $m / M=0.5$ and $m / M=0.1$ ). One observes that the number of collisions required to get into the thermal cloud increases as $m / M$ gets smaller, while the width of the cloud decreases.

$$
\tilde{L}_{B}(\mathrm{U} ; \mathbf{K})=\sqrt{\frac{\Gamma_{\beta}}{\sqrt{\pi} K}} \exp \left(-\frac{1}{2}\left(\frac{K}{2}+\frac{\mathrm{U} \cdot \mathbf{K}}{K}\right)^{2}\right) .
$$

We assume that the initial state is a momentum eigenstate $|\mathbf{U}\rangle$, labeled by the dimensionless momentum (4.51) of the test particle. The algorithm considered in Sect. 4.3.2 then leads to a pure jump process in $\mathbf{U}$, whose realizations can be obtained by means of the standard algorithm used for the stochastic simulation of classical Markovian master equations [107. This is due to the fact that the deterministic evolution given by Eq. (4.39) only affects the normalization in this case, since both $\mathrm{H}$ and $\mathrm{G}$ are only functions of the momentum operator.

According to Eq. (4.40) and Eq. (4.43) the waiting time distribution for a jump is characterized by the cumulative distribution function $F(\tau)=1-\exp [-\tilde{\Gamma}(U) \tau]$ in our case, where $\tilde{\Gamma}(U)=\tilde{M}_{\text {out }}^{\text {cl }}(\mathbf{U})$ is the dimensionless version of the loss rate. According to Eq. (4.45) and Eq. (4.48) it reads

$$
\tilde{\Gamma}(U)=\Gamma_{\beta} \frac{2}{\sqrt{\pi}}{ }_{1} F_{1}\left(-\frac{1}{2}, \frac{3}{2} ;-U^{2}\right),
$$

with $\Gamma_{\beta}$ as in Eq. (4.28) and ${ }_{1} F_{1}$ as in Eq. (4.49).

Each jump is characterized by a momentum transfer drawn from the probability density (4.42). In the present case, this distribution depends only on the modulus $K$ of the momentum transfer and on the cosine $\xi=\mathbf{U} \cdot \mathbf{K} /(U K)$ of the angle between $\mathbf{U}$ and $\mathbf{K}$,

$$
\mathcal{P}(K, \xi)=\frac{\Gamma_{\beta}}{2 \sqrt{\pi} \tilde{\Gamma}(U)} K \exp \left[-\left(\frac{K}{2}+U \xi\right)^{2}\right],
$$

while components of the momentum transfer perpendicular to $\mathbf{U}$ are uniformly distributed. For each jump one has to draw random numbers $(K, \xi)$ according to the probability density (4.55) and perform the shift 

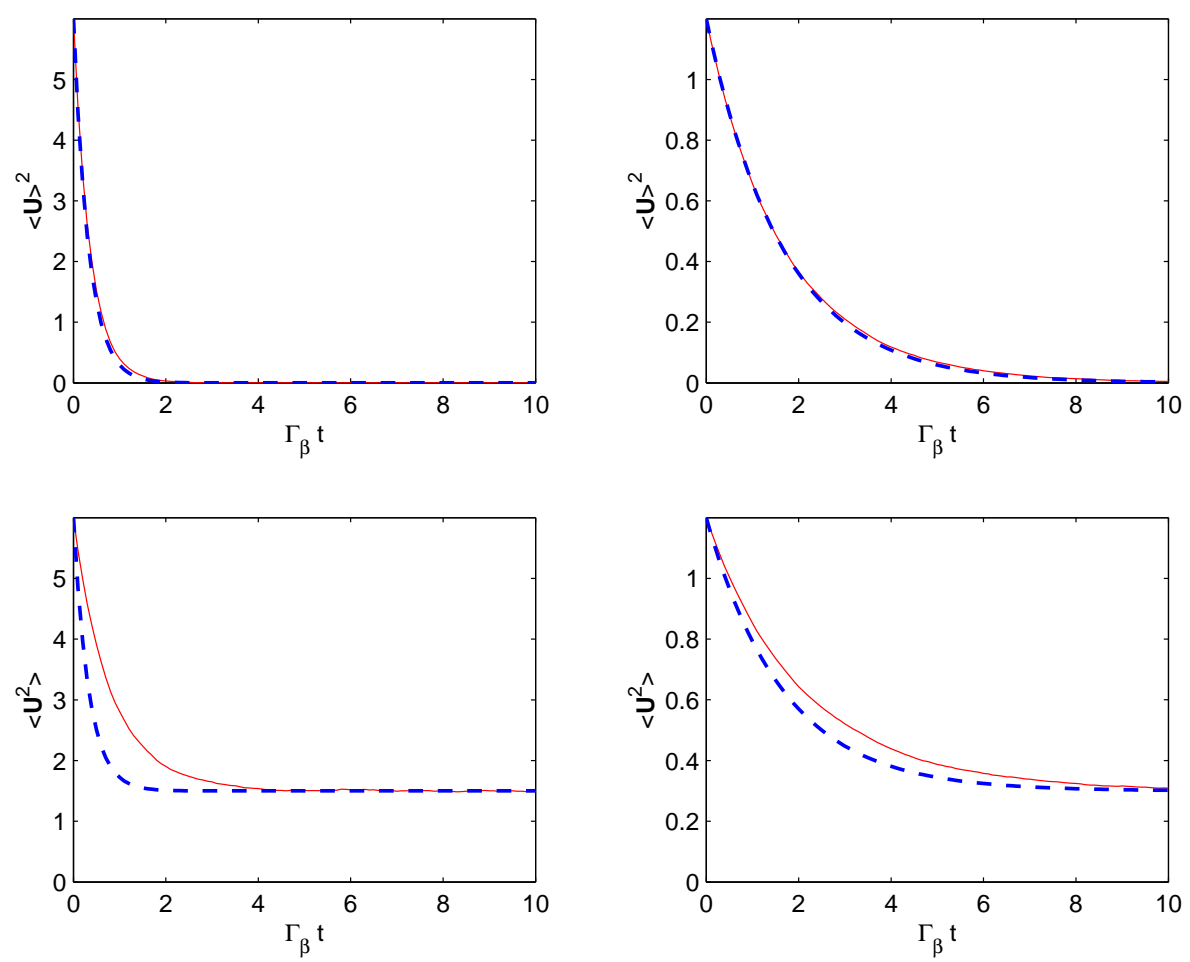

Fig. 4.2. (Color online) Averages of squared mean momentum (top) and mean squared momentum (bottom) for the mass ratios $m / M=1$ (left) and $m / M=0.2$ (right). The solid line gives the results obtained from the Monte Carlo method, while the dashed lines correspond to the analytic predictions in Eq. 4.57) and Eq. 4.58, respectively. As one expects, the analytic approximation deteriorates if one increases the mass ratio (left panels). In this regime the decay rates for the (squared) momentum and the kinetic energy also no longer coincide.

$\mathbf{U} \rightarrow \mathbf{U}+\mathbf{K} m_{*} / M$ with $\mathbf{K}$ given by

$$
\mathbf{K}=K \xi \frac{\mathbf{U}}{U}+K \sqrt{1-\xi^{2}} \frac{\mathbf{U} \times \mathbf{e}}{|\mathbf{U} \times \mathbf{e}|},
$$

with e a random unit vector.

Single realizations of the process for different values of the mass ratio are shown in Fig. 4.1 as threedimensional plots. One observes that the ratio between the masses of the gas and test particle affects the number of collisions which are necessary in order to drive the test particle momentum close to its equilibrium value. At smaller mass ratios $m / M$, an increasingly large number of collisions is required to thermalize the test particle. The fluctuations around the equilibrium value, on the other hand, decrease in this limit.

By averaging over a sufficiently large number of realizations one can study the relaxation of the momentum and of the kinetic energy of the test particle. According to the scaling introduced in Eq. (4.51) the expected equilibrium value $3 /(2 \beta)$ of the kinetic energy corresponds to $\left\langle\mathbf{U}^{2}\right\rangle_{\mathrm{eq}}=3 \mathrm{~m} /(2 M)$, while the momentum should relax to $\langle\mathbf{U}\rangle_{\mathrm{eq}}=0$. This behavior is confirmed well by the numerical simulations reported in Fig. 4.2. where the squared mean momentum $\langle\mathbf{U}\rangle_{\text {eq }}^{2}$ and the mean squared momentum $\left\langle\mathbf{U}^{2}\right\rangle_{\text {eq }}$ are plotted as a function of time in units of the inverse of the total scattering rate $\Gamma_{\beta}$.

The numerical results in Fig. 4.2 are compared to the analytic predictions Eq. (4.31) and Eq. (4.32) for the mean momentum and mean kinetic energy respectively, which are valid for $m / M \ll 1$. In terms of the dimensionless variable $\mathbf{U}$ the solutions of Eq. (4.31) and Eq. (4.32) read as

$$
\langle\mathbf{U}\rangle_{\rho_{t}}^{2}=\langle\mathbf{U}\rangle_{\rho_{0}}^{2} \mathrm{e}^{-2 \eta t}
$$

and 


$$
\left\langle\mathbf{U}^{2}\right\rangle_{\rho_{t}}=\left\langle\mathbf{U}^{2}\right\rangle_{\text {eq }}+\left[\left\langle\mathbf{U}^{2}\right\rangle_{\rho_{0}}-\left\langle\mathbf{U}^{2}\right\rangle_{\mathrm{eq}}\right] \mathrm{e}^{-2 \eta t},
$$

with $\eta$ given by Eq. (4.33). As one observes from Fig. 4.2 the analytic approximation is indeed very good for small mass ratios $m / M \ll 1$, while deviations appear for higher values of the ratio. These changes show up in particular through the appearance of different decay rates for the momentum and the kinetic energy. For large $m / M$ one can also observe strong deviations from Gaussian statistics, e.g., by studying the behavior of cumulants of order higher than two [101.

\section{The limit of quantum Brownian motion}

One of the most important limits of the quantum linear Boltzmann equation is the situation where the test particle has a much greater mass than the gas particles. In the classical case, this limit turns the linear Boltzmann equation into the Kramers equation for the description of Brownian motion. As discussed in the following, the situation is quite similar in the quantum case, where one obtains a quantum Fokker-Planck equation describing quantum Brownian motion.

The term quantum Brownian motion is generally used for master equations intended to phenomenologically describe the quantum analog of classical Brownian motion 108 109/110111, without accounting for the microscopic details of the underlying dynamics. In contrast to such models, which usually assume a linear coupling to a harmonic bath, we focus in the following on the specific situation of a massive test particle suffering collisions with a surrounding Maxwell-Boltzmann gas. The microscopic expressions of the friction and diffusion coefficients are worked out as a function of the cross-section, and we put into evidence the new quantum terms appearing in the master equation corresponding to the Kramers equation.

\subsection{The classical Kramers equation}

In order to discuss the Brownian motion limit of the quantum linear Boltzmann equation it is useful to first recall the relationship between the classical linear Boltzmann equation and Kramers' Fokker-Planck equation. It was Rayleigh who first investigated the limiting case of a very heavy test particle in the collision integral of the linear Boltzmann equation [112113]. The resulting equation in this so-called Rayleigh limit, or Brownian limit, is the well-known Kramers equation. For the homogeneous case and in the absence of an external force it reads as

$$
\partial_{t} f(\mathbf{P})=\eta \sum_{i=1}^{3}\left[\frac{\partial}{\partial P_{i}}\left(P_{i} f(\mathbf{P})\right)+\frac{M}{\beta} \frac{\partial^{2}}{\partial P_{i}^{2}} f(\mathbf{P})\right] .
$$

The friction coefficient $\eta$ is given by 1141115

$$
\eta=\frac{16}{3} \sqrt{\pi} \frac{m}{M} n_{\text {gas }} \sqrt{\frac{2}{\beta m}} \int_{0}^{\infty} \mathrm{d} u u^{5} \mathrm{e}^{-u^{2}} \int_{0}^{\pi} \mathrm{d} \vartheta \sin \vartheta(1-\cos \vartheta) \sigma\left(\vartheta ; u p_{\beta}\right),
$$

with $\sigma\left(\vartheta ; u p_{\beta}\right)$ the differential scattering cross-section as a function of the scattering angle and of the momentum of the gas particle $p=u p_{\beta}$.

The derivation of the microscopic expression of the friction coefficient is not easy to find in the literature. We therefore briefly recall the derivation of (5.1) and (5.2) in Sect. A.3 for the reader's convenience and for the sake of comparison with the quantum calculation. The crucial assumption required to derive the Fokker-Planck Eq. (5.1) from the classical linear Boltzmann equation (3.1) is that the test particle is much heavier than the gas particles, $m / M \ll 1$, and that its momentum is close to the typical thermal value $P_{\beta}=\sqrt{2 M / \beta}$. The collisions with the gas particles are then characterized by small momentum and energy transfers. The typical value of the momentum transfer $Q$ is of the order of the momentum of the colliding gas particles $p_{\beta}=\sqrt{2 m / \beta}$, and therefore much smaller than the momentum $P_{\beta}$ of the test particle, while the energy transfer is on the order of $E(\mathbf{Q}, \mathbf{P}) \approx 1 / \beta \sqrt{m / M}$, which is smaller than the thermal energy $1 / \beta$. As discussed in Sect. A.3, under these conditions one can expand $f\left(\mathbf{P}^{\prime}\right)$ in Eq. (3.1) around $\mathbf{P}$ for small momentum transfers $\mathbf{Q}=\mathbf{P}^{\prime}-\mathbf{P}$, and arrive at Eq. (5.1) by sorting the orders in an $m / M$ expansion. 


\subsection{The quantum Brownian limit}

\subsubsection{Conditions of validity}

The situation is more complicated in the quantum case, since one is dealing with operators whose values can be estimated in a meaningful way only by considering suitable matrix elements. We will show that the quantum master equation which corresponds to the classical Fokker-Planck equation is obtained by expanding the scattering kernel up to second order in the operators $\mathrm{X}$ and $\mathrm{P}$. It is important to stress that the result is not equivalent to a direct application of the correspondence principle on the classical result, which would lead to a master equation which does not generally preserve the positivity of the statistical operator 116117.

The expansion can be performed provided the test particle is very massive and not far from thermal equilibrium, that is to say, close to diagonal in momentum representation. More precisely, the off-diagonal elements $\left\langle\mathbf{P}|\rho| \mathbf{P}^{\prime}\right\rangle$ may differ significantly from zero only for $\Delta P:=\left|\mathbf{P}-\mathbf{P}^{\prime}\right| \lesssim \sqrt{M / \beta}$. In the position representation, the validity of the expansion requires that the motional state exhibits coherences only over a length scale of the order of the thermal wavelength of the test particle, such that $\left\langle\mathbf{X}|\rho| \mathbf{X}^{\prime}\right\rangle$ is appreciably different from zero only within a range given by the thermal de Broglie wavelength $\lambda_{\text {th }}=\sqrt{2 \pi \hbar^{2} \beta / M}$, i.e., for $\Delta X:=\left|\mathbf{X}-\mathbf{X}^{\prime}\right| \lesssim \lambda_{\text {th }}$. The small mass ratio $m / M \ll 1$ implies in particular that when the test particle gets close to thermal equilibrium its coherence length of the order of its thermal de Broglie wavelength is much smaller than the coherence length of the environment given by the thermal wavelength of the gas.

\subsubsection{Diffusive limit of the quantum linear Boltzmann equation}

In order to study the Brownian motion limit of the quantum linear Boltzmann equation, we start with the incoherent part given by Eq. (2.2). Using the explicit expression of the Lindblad operators Eq. (2.3) with the distribution function (2.6) of a Maxwell-Boltzmann gas, one obtains the exact expression

$$
\begin{aligned}
\mathcal{L} \rho= & n_{\text {gas }} \frac{m}{m_{*}^{2}} \int \frac{\mathrm{d} \mathbf{Q}}{Q} \int_{\mathbf{Q}^{\perp}} \mathrm{d} \mathbf{k}_{\perp} \mu_{\beta}\left(\mathbf{k}_{\perp}+\frac{m}{m_{*}} \frac{\mathbf{Q}}{2}\right)\left[f\left(\operatorname{rel}\left(\mathbf{k}_{\perp}, \mathbf{P}_{\perp \mathbf{Q}}\right)-\frac{\mathbf{Q}}{2}, \operatorname{rel}\left(\mathbf{k}_{\perp}, \mathbf{P}_{\perp \mathbf{Q}}\right)+\frac{\mathbf{Q}}{2}\right)\right. \\
& \times \mathrm{e}^{i \mathbf{Q} \cdot \mathbf{x} / \hbar} \exp \left(-\beta \frac{m \mathbf{P}_{\| \mathbf{Q}}^{2}}{4 M^{2}}-\beta \frac{m \mathbf{Q} \cdot \mathbf{P}}{4 M m_{*}}\right) \rho \exp \left(-\beta \frac{m \mathbf{P}_{\| \mathbf{Q}}^{2}}{4 M^{2}}-\beta \frac{m \mathbf{Q} \cdot \mathbf{P}}{4 M m_{*}}\right) \mathrm{e}^{-i \mathbf{Q} \cdot \mathbf{x} / \hbar} \\
& \times f^{\dagger}\left(\operatorname{rel}\left(\mathbf{k}_{\perp}, \mathrm{P}_{\perp \mathbf{Q}}\right)-\frac{\mathbf{Q}}{2}, \operatorname{rel}\left(\mathbf{k}_{\perp}, \mathrm{P}_{\perp \mathbf{Q}}\right)+\frac{\mathbf{Q}}{2}\right) \\
& \left.-\frac{1}{2}\left\{\exp \left(-\beta \frac{m \mathrm{P}_{\| \mathbf{Q}}^{2}}{4 M^{2}}-\beta \frac{m \mathbf{Q} \cdot \mathbf{P}}{4 M m_{*}}\right)\left|f\left(\operatorname{rel}\left(\mathbf{k}_{\perp}, \mathrm{P}_{\perp \mathbf{Q}}\right)-\frac{\mathbf{Q}}{2}, \operatorname{rel}\left(\mathbf{k}_{\perp}, \mathbf{P}_{\perp \mathbf{Q}}\right)+\frac{\mathbf{Q}}{2}\right)\right|^{2}, \rho\right\}\right]
\end{aligned}
$$

In the expansion up to second order in $\mathrm{P}$ the contributions from the terms $\exp \left(-\beta m \mathrm{P}_{\| \mathbf{Q}}^{2} /\left(4 M^{2}\right)\right)$ cancel out, such that they can be replaced by unity. We further expand the various quantities under the assumption that the change in momentum of the Brownian particle is small compared to the scales involved in its motional state. More specifically, one has to assume that for typical values of the momentum transfer $Q$ the relevant matrix elements of the statistical operator vanish unless

$$
\frac{Q}{\hbar} \Delta X \ll 1
$$

and

$$
\frac{\beta Q}{M} \Delta P \ll 1
$$

These conditions are both satisfied if the Brownian state is close to thermal and $M \gg m$, such that $m_{*} \approx m$, since the momentum transfer $Q$ is then typically of the order of the momentum $p_{\beta}=\sqrt{2 m / \beta}$ of the colliding gas particles. This implies in particular that 


$$
\frac{Q}{\hbar} \approx \sqrt{\frac{m}{2 \pi \hbar^{2} \beta}}=\frac{1}{\lambda_{\mathrm{th}}^{\text {gas }}} \ll \frac{1}{\lambda_{\mathrm{th}}} \lesssim \frac{1}{\Delta X}
$$

and

$$
\frac{\beta Q}{M} \approx \sqrt{\frac{m}{M}} \sqrt{\frac{\beta}{M}} \ll \sqrt{\frac{\beta}{M}} \lesssim \frac{1}{\Delta P} .
$$

Apart from the exponentials in Eq. (5.3) one also has to expand the scattering amplitude, which appears as an operator-valued expression. However, for the assumed small mass ratio the argument of the scattering amplitude becomes

$$
\operatorname{rel}\left(\mathbf{k}_{\perp}, \mathbf{P}_{\perp \mathbf{Q}}\right)-\frac{\mathbf{Q}}{2} \approx \mathbf{k}_{\perp}-\frac{m}{M} \mathbf{P}_{\perp \mathbf{Q}}-\frac{\mathbf{Q}}{2}
$$

such that the leading term in an expansion in $m / M$ does not depend any longer on $\mathbf{P}_{\perp \mathbf{Q}}$. This removes an operator dependence arising from the scattering amplitude. By further expanding the exponentials involving $\mathrm{Q} \cdot \mathrm{P}$ in Eq. (5.3) one is then left with

$$
\begin{aligned}
\mathcal{L} \rho= & -\frac{n_{\text {gas }}}{m} \frac{1}{2} \int \frac{\mathrm{d} \mathbf{Q}}{Q} \sum_{j, k=1}^{3} Q_{j} Q_{k} \int_{\mathbf{Q}^{\perp}} \mathrm{d} \mathbf{k}_{\perp} \mu_{\beta}\left(\mathbf{k}_{\perp}+\frac{\mathbf{Q}}{2}\right)\left|f\left(\mathbf{k}_{\perp}-\frac{\mathbf{Q}}{2}, \mathbf{k}_{\perp}+\frac{\mathbf{Q}}{2}\right)\right|^{2} \\
& \times\left[\frac{1}{\hbar^{2}}\left[\mathbf{X}_{j},\left[\mathbf{X}_{k}, \rho\right]\right]+\left(\frac{\beta}{4 M}\right)^{2}\left[\mathrm{P}_{j},\left[\mathrm{P}_{k}, \rho\right]\right]+\frac{i}{\hbar} \frac{\beta}{2 M}\left[\mathbf{X}_{j},\left\{\mathrm{P}_{k}, \rho\right\}\right]\right] .
\end{aligned}
$$

We here omitted the term linear in the momentum transfer, since it vanishes upon integration due to the isotropy of the integrand. By exploiting also the invariance of the scattering cross-section under parity transformations the quantity $Q_{j} Q_{k}$ can be replaced by $\delta_{j k} Q^{2} / 3$. We have thus put into evidence the operator structure of the limiting expression of the quantum linear Boltzmann equation for the case of quantum Brownian motion,

$$
\mathcal{L} \rho=-\eta \sum_{j=1}^{3}\left[\frac{M}{\hbar^{2} \beta}\left[\mathbf{X}_{j},\left[\mathbf{X}_{j}, \rho\right]\right]+\frac{\beta}{16 M}\left[\mathrm{P}_{j},\left[\mathrm{P}_{j}, \rho\right]\right]+\frac{i}{2 \hbar}\left[\mathbf{X}_{j},\left\{\mathrm{P}_{j}, \rho\right\}\right]\right] .
$$

This equation is still covariant under translations [47/56/93], since the limiting procedure does not spoil the symmetry properties.

\subsubsection{Microscopic expression for the friction constant}

We now evaluate the friction coefficient $\eta$ in Eq. (5.10), which is defined by

$$
\eta=\frac{\beta}{6} \frac{n_{\text {gas }}}{M m} \int \mathrm{d} \mathbf{Q} Q \int_{\mathbf{Q}^{\perp}} \mathrm{d}_{\mathbf{k}_{\perp}} \mu_{\beta}\left(\mathbf{k}_{\perp}+\frac{\mathbf{Q}}{2}\right)\left|f\left(\mathbf{k}_{\perp}-\frac{\mathbf{Q}}{2}, \mathbf{k}_{\perp}+\frac{\mathbf{Q}}{2}\right)\right|^{2} .
$$

The exact expression of $\eta$ will turn out to be the same as the classical friction coefficient (5.2). Comparing the procedure followed here with the calculations in Appendix A.3 it turns out that the microscopic derivation of the friction coefficient is more straightforward in the quantum case. Upon using $\delta(\mathbf{p} \cdot \mathbf{Q})=\delta\left(p_{\|}\right) / Q$ and introducing the new variables $\mathbf{p}_{i}=\mathbf{p}+\mathbf{Q} / 2$ and $\mathbf{p}_{f}=\mathbf{p}-\mathbf{Q} / 2$ the expression for $\eta$ becomes

$$
\eta=\frac{\beta}{6} \frac{n_{\text {gas }}}{M m} \int \mathrm{d} \mathbf{p}_{i} \int \mathrm{d} \mathbf{p}_{f} \delta\left(\frac{p_{i}^{2}-p_{f}^{2}}{2}\right)\left|\mathbf{p}_{i}-\mathbf{p}_{f}\right|^{2} \mu_{\beta}\left(\mathbf{p}_{i}\right)\left|f\left(\mathbf{p}_{f}, \mathbf{p}_{i}\right)\right|^{2} .
$$

As a final step, we introduce the angle $\vartheta$ between $\mathbf{p}_{i}$ and $\mathbf{p}_{f}$ as an integration variable, and recall that the scattering cross-section is assumed to depend only on $p_{i}$ and $\vartheta$, thus finally obtaining for a MaxwellBoltzmann gas distribution 


$$
\eta=\frac{16}{3} \sqrt{\pi} \frac{m}{M} n_{\text {gas }} \sqrt{\frac{2}{m \beta}} \int_{0}^{\infty} \mathrm{d} u u^{5} \mathrm{e}^{-u^{2}} \int_{0}^{\pi} \mathrm{d} \vartheta \sin \vartheta(1-\cos \vartheta)\left|f\left(\vartheta ; u p_{\beta}\right)\right|^{2},
$$

with $u$ as in (5.2). This expression coincides with the classical result [114, recalled in Eq. (5.2). For the case of a constant scattering cross-section it reads as

$$
\eta=\frac{8}{3 \sqrt{\pi}} \frac{m}{M} \Gamma_{\beta},
$$

which also agrees with the result (4.33) obtained in Sect. 4.3

\subsection{The master equation of quantum Brownian motion}

Putting the master equation for quantum Brownian motion Eq. (5.10) in the standard form, it finally reads

$$
\mathcal{L} \rho=\frac{1}{i \hbar} \frac{\eta}{2} \sum_{j=1}^{3}\left[\mathrm{X}_{j},\left\{\mathrm{P}_{j}, \rho\right\}\right]-\frac{D_{p p}}{\hbar^{2}} \sum_{j=1}^{3}\left[\mathrm{X}_{j},\left[\mathrm{X}_{j}, \rho\right]\right]-\frac{D_{x x}}{\hbar^{2}} \sum_{j=1}^{3}\left[\mathrm{P}_{j},\left[\mathrm{P}_{j}, \rho\right]\right] .
$$

The friction coefficient $\eta$ is given by Eq. (5.13), while $D_{p p}$ has the meaning of a diffusion coefficient,

$$
D_{p p}=\eta \frac{M}{\beta},
$$

and $D_{x x}$ is related to $D_{p p}$ and to $\eta$ by

$$
D_{x x}=\left(\frac{\beta \hbar}{4 M}\right)^{2} D_{p p}=\frac{\beta \hbar^{2}}{16 M} \eta .
$$

This equation admits an exact solution, though the explicit expression is a bit cumbersome [118]. We shall consider it in Sect. 7.4 when studying how this master equation describes decoherence effects.

We note that the Caldeira-Leggett master equation, describing the high-temperature regime of the CaldeiraLeggett model 119]120[121|111], is related to Eq. (5.15) by neglecting the third term in (5.15), i.e., by setting $D_{x x}=0$. This equation, which cannot be brought into Lindblad form, is also obtained from the classical Kramers equation if one takes the naive operator correspondence $\partial / \partial P_{j} \rightarrow \mathbf{X}_{j} /(i \hbar)$.

\subsubsection{The diffusion coefficients and complete positivity}

The diffusion coefficient $D_{p p}$ can also be related to the second moment of the thermal momentum $\left\langle P^{2}\right\rangle_{\beta}=$ $3 M / \beta$ according to

$$
D_{p p}=\frac{\eta}{3}\left\langle P^{2}\right\rangle_{\beta},
$$

while $D_{x x}$, which is sometimes denoted as "quantum diffusion coefficient" or "momentum localization coeffi-

cient", can be written by means of the thermal de Broglie wavelength $\lambda_{\text {th }}=\sqrt{2 \pi \hbar^{2} \beta / M}$ of the test particle as

$$
D_{x x}=\frac{\eta}{32 \pi} \lambda_{\mathrm{th}}^{2} .
$$

As discussed in [122[123|124|125]126|29] a necessary and sufficient condition for master equations of the form (5.15) to yield a completely positive dynamics is

$$
D_{x x} D_{p p} \geqslant \frac{\eta^{2} \hbar^{2}}{16}
$$

since only then they can be cast into Lindblad form. This is an important statement, because in many approaches the coefficients $\eta, D_{x x}$, and $D_{p p}$ are treated as free, phenomenological parameters. In contrast 
to that, the derivation from the quantum linear Boltzmann equation yielded microscopically formulated definitions and relations of the coefficients (5.15)-(5.17). It is therefore reassuring that $D_{x x}$ and $D_{p p}$ from Eq. (5.17) and Eq. (5.16) satisfy Eq. (5.20) with an equality sign.

For a given choice of $\eta$ and $D_{p p}=\eta M / \beta$ the coefficient $D_{x x}$ obtained in Eq. (5.17) therefore takes the minimal value allowed within the framework of a Lindblad master equation. This implies that the equation of quantum Brownian motion obtained from the quantum linear Boltzmann equation is as close to the classical Kramers equation as it can possibly be, within the bounds set by the requirement of complete positivity.

\subsubsection{The quantum diffusion term}

It was discussed by several authors [122]123|124|125|127/29] that the lack of complete positivity of the Caldeira Leggett master equation can be corrected by adding a "position diffusion term" of the form $\left[\mathrm{P}_{j},\left[\mathrm{P}_{j}, \rho\right]\right]$. In view of the fact that the Brownian limit of the quantum linear Boltzmann equation yields the minimal correction, it is worth noting that this is not achieved in other microscopic approaches.

Diòsi's proposal [44, for instance, leads to an expression of the coefficient $D_{x x}$, whose relation to $D_{p p}$ is not given exclusively by thermodynamic quantities. Rather, it involves a complicated average over the scattering cross-section. More refined derivations within the Caldeira Leggett model, on the other hand, which seek for correction terms beyond the high temperature approximation 128]129, lead to a coefficient $D_{x x}$ which exceeds the present, minimal value by a factor of $4 / 3$.

As already mentioned, the term $\sum_{j=1}^{3}\left[\mathrm{P}_{j},\left[\mathrm{P}_{j}, \rho\right]\right]$ in Eq. (5.15) has no counterpart in the classical FokkerPlanck equation (5.1). It describes a position diffusion process, which is expected to appear together with momentum diffusion, as implied by the inequality Eq. (5.20). That this is a pure quantum phenomenon is confirmed by the fact that the coefficient $D_{x x}$ depends explicitly on $\hbar$; it vanishes in the semiclassical limit $\hbar \rightarrow 0$.

Even though the occurrence of this quantum effect leading to momentum localization was predicted in many theoretical papers, it is still unclear how to observe the effect experimentally. The main difficulty lies in the fact that the position localization term, with its direct classical analog, dominates under typical experimental conditions, due to its strong temperature dependence, while the quantum diffusion term vanishes in the limit of high temperatures. Effects of the latter might show up by either inducing decoherence with respect to the momentum basis, or by looking for corrections to the classical diffusion coefficient. The last possibility was considered for the strong friction limit of the quantum master equation [130. Compared to the classical case, the Einstein diffusion coefficient appears multiplied by a factor $1+(\eta \beta \hbar)^{2} / 16$, involving the ratio between two characteristic times $\beta \hbar$ for the bath of gas particles and $1 / \eta$ for the system. The separation of time scales typical for a Markov description of the dynamics implies that this correction should be extremely small under typical conditions.

\section{Matter wave optics in gaseous samples}

So far, we mostly dealt with features of the quantum linear Boltzmann equation that are also shared by its classical counterpart. In particular, its symmetry and relaxation properties, as well as the existence of the stationary solution, are already described by the behavior of the momentum populations, i.e., of the diagonal matrix elements of Eq. (2.7). In the following two sections, we will now consider properties of the quantum linear Boltzmann equation that become manifest in interference phenomena, where it is decisive that the motion of the test particle may be in a coherently delocalized, wavelike state.

An important quantum effect that one expects to be described by Eq. (2.2) is the dynamical transition of an initially delocalized superposition state into an incoherent, particlelike mixture. Such decoherence phenomena are described in Sect. 7 But before that, we focus on the dynamics described by the coherent part of the quantum linear Boltzmann equation and its loss term. They play an important role in experiments involving spatially separate beams.

The claim that massive particles can exhibit wave-like behavior is one of the central predictions of quantum mechanics; it is confirmed experimentally with neutrons [131132], atoms [133/134]35], and even complex molecules [135]. In such experiments, the de Broglie wave length $\lambda_{\mathrm{dB}}=h /(M V)$ of the matter waves is 
usually much shorter than the scale of change of the potential produced by the gratings and collimation slits, which act as optical elements. It is then often permissible to neglect diffraction phenomena by describing the matter wave in terms of well defined beams, which are coherently split and rejoined by means of interference gratings. The effect of an additional external potential on those beams can then be accounted for, in complete analogy to the optical case, by introducing an index of refraction.

In a number of Mach-Zehnder-type interference experiments a truly macroscopic separation of the interfering beams was achieved. This makes it possible to perform experiments where the interfering particles interact with an external system in just one arm of the interferometer. The modification of the interference fringe pattern then provides sensitive information of the strength of interaction. Its theoretical description requires the use of quantum kinetic equations if the interaction is due to a dilute background gas, as realized in experiments with sodium and lithium atoms 3713638, or due to a homogeneous sample of thermalized condensed matter, as in the experiments performed with the perfect crystal neutron interferometer [137132].

\subsection{The gas induced energy shift}

The presence of a background gas manifests itself not only in the incoherent part $\mathcal{L}$ of the quantum linear Boltzmann equation, but also in a modified Hamiltonian $\mathrm{H}=\mathrm{P}^{2} /(2 M)+H_{\mathrm{n}}(\mathrm{P})$ appearing in its coherent part. The additional term $H_{\mathrm{n}}(\mathrm{P})$, which has been neglected so far, describes the modification of the free matter wave dispersion relation due to the coherent interaction with the background gas. It becomes observable in the abovementioned Mach-Zehnder interferometers as a gas-induced phase shift.

According to Eq. (2.5), derived in 32, the energy shift $H_{\mathrm{n}}(\mathrm{P})$ is related to a "forward scattering process" which does not change the momenta of the gas and the test particle. The forward scattering amplitude $f_{0}(\mathbf{p}) \equiv f(\mathbf{p}, \mathbf{p})$ appears in a thermal average over the momentum distribution of the gas,

$$
\left\langle f_{0}(\mathbf{P})\right\rangle_{\mu}=\int \mathrm{d} \mathbf{p} \mu(\mathbf{p}) f(\operatorname{rel}(\mathbf{p}, \mathbf{P}), \operatorname{rel}(\mathbf{p}, \mathbf{P})),
$$

allowing us to write more compactly

$$
H_{\mathrm{n}}(\mathrm{P})=-2 \pi \hbar^{2} \frac{n_{\text {gas }}}{m_{*}} \operatorname{Re}\left\langle f_{0}(\mathrm{P})\right\rangle_{\mu}
$$

To observe the effect of Eq. (6.2) an experimental situation is required where the interfering matter wave beams are strongly collimated and directed into narrow apertures. In this case any proper, momentum changing collision due to a dilute background gas will unavoidably lead to a loss of the particle. This implies that all particles arriving in the detector experienced at most the effect of "forward scattering". It can be observed as a phase shift in the interference pattern, as done in the experiments [37 136 38].

If the particles do not get lost, their motional state is modified at most coherently in the described experimental situation. The purity of the matter waves impinging at the detector therefore remains unchanged by the presence of the background gas, rendering a description by means of a wave equation possible. The effect of particle loss can be accounted for by an attenuation of the amplitude, as argued below in Sect. 6.3. Incidentally, the loss term introduced in Eq. (2.13) and discussed in Sec. 4.3.3. can be related to the imaginary part of Eq. (6.1). This fact naturally leads to the introduction of an optical potential.

\subsection{The optical potential}

It is helpful to express the damping of the wave amplitude due to collisional losses in term of the forward scattering amplitude. Let us start from Eq. (3.1) for the loss term in the classical linear Boltzmann equation. Switching to center-of-mass variables with respect to $\mathbf{p}^{\prime}$ and $\mathbf{P}^{\prime}$, denoting $\mathbf{k}=\operatorname{rel}\left(\mathbf{p}^{\prime}, \mathbf{P}^{\prime}\right)$ and $\mathbf{K}=\mathbf{p}^{\prime}+\mathbf{P}^{\prime}$ one obtains

$$
M_{\text {out }}^{\mathrm{cl}}(\mathbf{P})=\frac{n_{\text {gas }}}{m_{*}} \int \mathrm{d} k k \int \mathrm{d} \mathbf{p} \mu(\mathbf{p}) \delta(k-|\operatorname{rel}(\mathbf{p}, \mathbf{P})|) \int \mathrm{d} \Omega_{\mathbf{k}} \sigma(\mathbf{k}, \operatorname{rel}(\mathbf{p}, \mathbf{P})) .
$$


We also introduce the total scattering cross-section

$$
\sigma_{\text {tot }}(\mathbf{p})=\int \mathrm{d} \Omega_{\mathbf{n}} \sigma(p \mathbf{n}, \mathbf{p}),
$$

where $\mathbf{n}$ is a unit vector with $\mathrm{d} \Omega_{\mathbf{n}}$ the associated solid angle element. By exploiting the optical theorem [138,

$$
\sigma_{\text {tot }}(\mathbf{p})=\frac{4 \pi \hbar}{p} \operatorname{Im}[f(\mathbf{p}, \mathbf{p})],
$$

and by using the thermal average defined in Eq. (6.1), one finds that the loss term takes the simple form

$$
M_{\text {out }}^{\mathrm{cl}}(\mathbf{P})=\frac{n_{\text {gas }}}{m_{*}} 4 \pi \hbar \operatorname{Im}\left\langle f_{0}(\mathbf{P})\right\rangle_{\mu} .
$$

Recalling Eq. (2.7) and Eq. (6.2) the quantum linear Boltzmann equation can therefore be written as

$$
\begin{aligned}
\frac{\mathrm{d}}{\mathrm{d} t} \rho= & -\frac{i}{\hbar}\left[\mathrm{H}_{0}-2 \pi \hbar^{2} \frac{n_{\text {gas }}}{m_{*}} \operatorname{Re}\left\langle f_{0}(\mathrm{P})\right\rangle_{\mu}, \rho\right]-\frac{1}{2}\left\{\frac{n_{\text {gas }}}{m_{*}} 4 \pi \hbar \operatorname{Im}\left\langle f_{0}(\mathrm{P})\right\rangle_{\mu}, \rho\right\} \\
& +\int \mathrm{d} \mathbf{Q} \int_{\mathbf{Q}^{\perp}} \mathrm{d} \mathbf{k}_{\perp} \mathrm{e}^{i \mathbf{Q} \cdot \mathbf{X} / \hbar} L\left(\mathbf{k}_{\perp}, \mathrm{P} ; \mathbf{Q}\right) \rho L^{\dagger}\left(\mathbf{k}_{\perp}, \mathrm{P} ; \mathbf{Q}\right) \mathrm{e}^{-i \mathbf{Q} \cdot \mathbf{X} / \hbar} .
\end{aligned}
$$

This suggests to introduce a non-hermitian operator, often denoted as optical potential, of the form

$$
V_{\text {opt }}(\mathrm{P})=-2 \pi \hbar^{2} \frac{n_{\text {gas }}}{m_{*}}\left\langle f_{0}(\mathrm{P})\right\rangle_{\mu} .
$$

One thus arrives at a compact and suggestive expression for the quantum linear Boltzmann equation

$$
\begin{aligned}
\frac{\mathrm{d}}{\mathrm{d} t} \rho= & \frac{1}{i \hbar}\left[\mathrm{H}_{0}, \rho\right]+\frac{1}{i \hbar}\left(V_{\text {opt }}(\mathrm{P}) \rho-\rho V_{\text {opt }}^{\dagger}(\mathrm{P})\right) \\
& +\int \mathrm{d} \mathbf{Q} \int_{\mathbf{Q}^{\perp}} \mathrm{d} \mathbf{k}_{\perp} \mathrm{e}^{i \mathbf{Q} \cdot \mathbf{X} / \hbar} L\left(\mathbf{k}_{\perp}, \mathrm{P} ; \mathbf{Q}\right) \rho L^{\dagger}\left(\mathbf{k}_{\perp}, \mathrm{P} ; \mathbf{Q}\right) \mathrm{e}^{-i \mathbf{Q} \cdot \mathbf{X} / \hbar} .
\end{aligned}
$$

The first three terms at the right-hand side describe a coherent dynamics. They preserve the pure states, but not their normalization, due to the fact that the optical potential is not self-adjoint. The completely positive mapping expressed by the last term describes an incoherent contribution. It transforms pure states into mixtures and accounts for the preservation of the trace.

\subsection{Index of refraction}

As described above, typical matter wave interference experiments are characterized by a well-collimated, stationary beam of particles, with a momentum centered around a reference value $\mathbf{P}=\hbar \mathbf{K}$ in the longitudinal direction. One can then exploit the formal analogy between the Schrödinger equation and the Helmholtz wave equation 131133135] in order to introduce an index of refraction for matter waves.

For the case of a constant, complex potential,

$$
V_{\mathrm{opt}}=-2 \pi \hbar^{2} \frac{n_{\mathrm{gas}}}{m_{*}}\left\langle f_{0}(\hbar \mathbf{K})\right\rangle_{\mu},
$$

the stationary Schrödinger equation takes the form

$$
\nabla^{2} \psi(\mathbf{r})+\frac{2 M}{\hbar^{2}}\left[E-V_{\mathrm{opt}}\right] \psi(\mathbf{r})=0 .
$$

By analogy with the Helmholtz equation one can introduce a wave number associated to the propagation of the scalar field $\psi(\mathbf{r})$ with energy $E$, 


$$
K^{\prime}=\sqrt{\frac{2 M}{\hbar^{2}}\left[E-V_{\mathrm{opt}}\right]} .
$$

The effect of the optical potential on the dynamics can thus be equivalently described by an effective index of refraction

$$
n=\frac{K^{\prime}}{K}=\sqrt{1-\frac{V_{\mathrm{opt}}}{E}},
$$

where $K=\sqrt{2 M E} / \hbar$ is the wave number associated to the de Broglie wavelength of the tracer particle. Since the optical potential can be non-Hermitian in general, the index of refraction is in general complex.

In the situations described above, where the last, incoherent term of Eq. (6.9) can be neglected, the matter wave dynamics can thus be effectively described by a wave equation with the optical potential Eq. (6.8). The beam state may then be equivalently expressed as a wave propagation in a medium characterized by the refractive index (6.13), in complete analogy to the optical case.

For dilute gases the complex index of refraction is very close to unity, such that

$$
n \approx 1+2 \pi \frac{n_{\text {gas }}}{K^{2}} \frac{M}{m_{*}}\left\langle f_{0}(\hbar \mathbf{K})\right\rangle_{\mu} .
$$

Its real part

$$
n_{1}=1+2 \pi \frac{n_{\text {gas }}}{K^{2}} \frac{M}{m_{*}} \operatorname{Re}\left\langle f_{0}(\hbar \mathbf{K})\right\rangle_{\mu},
$$

describes the phase shift due to the background gas, while its imaginary part

$$
\begin{aligned}
n_{2} & =2 \pi \frac{n_{\text {gas }}}{K^{2}} \frac{M}{m_{*}} \operatorname{Im}\left\langle f_{0}(\hbar \mathbf{K})\right\rangle_{\mu} \\
& =\frac{M_{\mathrm{cl}}^{\text {out }}(\hbar \mathbf{K})}{2 \hbar K^{2} / M}
\end{aligned}
$$

determined by the loss term of the classical linear Boltzmann equation, accounts for the attenuation of the coherent beam.

We stress that this analysis in terms of a refractive index relies on neglecting the last term in Eq. [6.9). This is only valid under very particular experimental conditions. These might be realized in a Mach-Zender interferometer provided just one of the spatially separated paths interacts with a background gas and particles whose momentum has changed due to this interaction are blocked by the interferometer apertures. In this case only the part of the incoming beam scattered in the forward direction contributes to the signal collected at the outlet of the interferometer. Note in particular that the preservation of the trace implies that the loss and gain term do have the same weight, such that one is not allowed to neglect the last term of Eq. (6.9) because of its smallness with respect to the anticommutator term.

At variance with the typical intuition developed for electromagnetic waves the imaginary part of the index of refraction, which describes the exponential decay of the beam intensity, does not involve true absorption. The tracer particles are obviously not absorbed by the background gas, but the coherent collimated part of the beam entering the interferometer and responsible for the final interference pattern gets reduced. This is the reason why the reduction of intensity in the signal does not lead to a corresponding reduction of the visibility as in the case of collisional decoherence 39. Over a distance $L$ travelled with velocity $V=P / M$ the reduction factor is given by the exponential $\exp \left(-M_{\mathrm{cl}}^{\text {out }}(\mathbf{P}) L / V\right)$. Comparing this quantity with the damped intensity $\exp \left(-2 n_{2} K L\right)$ of an electromagnetic wave with wavenumber $K$ in a medium characterized by an index of refraction with imaginary part $n_{2}$ gives indeed back Eq. (6.16).

Specializing to a rotationally invariant scattering amplitude, $f\left(\mathbf{p}_{f}, \mathbf{p}_{i}\right)=f\left(\vartheta ; E_{\text {rel }}\right)$, with $\vartheta$ the angle between incoming and outgoing relative momentum, and $E_{\mathrm{rel}}=p_{i}^{2} / 2 m_{*}$ the kinetic energy in the center of mass, one can explicitly evaluate the thermal average introduced in Eq. (6.1) 


$$
\left\langle f_{0}(\mathbf{P})\right\rangle_{\mu}=\frac{2}{\sqrt{\pi}} \int_{0}^{\infty} \frac{\mathrm{d} v}{v_{\beta}} \frac{v}{V} \sinh \left(\frac{2 v V}{v_{\beta}^{2}}\right) \exp \left(-\frac{v^{2}+V^{2}}{v_{\beta}^{2}}\right) f\left(0 ; \frac{m_{*} v^{2}}{2}\right) .
$$

Here $V=P / M$ is the velocity of the interfering particle and a Maxwell-Boltzmann distribution (2.6) is assumed with $v_{\beta}=p_{\beta} / m$.

This expression, derived here from the quantum linear Boltzmann equation [32, agrees with the formula in 139140, which was recently obtained along a very different line of thought. While it has been used in the most recent interferometric experiments with lithium atoms 38, it deviates from previous theoretical results 141142] used for the analysis of the earlier experiments [37136143]. The difference lies in the thermal averaging procedure employed, and the averages in Eqs. (6.1), (6.17), which follow naturally from the linear Boltzmann equation, should be considered correct, as also pointed out in [140]. We note that still different methods of deriving a gas induced index of refraction were recently presented in 79:144].

It was already emphasized that the notion of a gas induced index of refraction can only be applied to a restricted set of experimental situations. One may describe phase shift and particle loss phenomena with this concept, but it does not apply to proper decoherence phenomena, where the purity of the motional state in the beams gets dynamically reduced due to the interaction with a background gas. As described in the following section, we have to return to a density matrix description in order to account for such effects.

\section{Collisional decoherence of matter waves}

The concept of environmental decoherence [145|146|147|148|149|29] is often drawn upon to explain how the emergence of classical behavior comes about if physical systems get larger and more complex. Though formulated in the same framework of open quantum systems also used to describe friction and thermalization, decoherence should be distinguished from such dissipative phenomena since it usually takes place on much shorter time scales and even in the absence of relaxation processes with a classical analog.

The main point is to acknowledge the crucial role played by the quantum correlations arising between the system and environmental degrees of freedom. The combined state of system and environment is no longer separable after the interaction, resulting in a loss of purity in the reduced quantum state, which is obtained by disregarding the environmental state by means of a partial trace. In a complementary view, which highlights the quantum aspects of the correlation, one may view the interaction as constituting an information transfer from the system to the environment.

In our case of a quantum test particle, collisions with a background gas have the effect of unsharp position measurements because the scattered state of a gas particle carries away some information on the position of the tracer which would be available, in principle, to an outside observer. One therefore expects that if the test particle is initially in a superposition of two positions, as in an interferometer, the coherence of this state will be reduced the more the better a typical scattering event can distinguish between the two positions. Once the coherence expressed by the off-diagonal elements in the position representation has vanished, the motional state will be indistinguishable from a classical mixture.

In the following we discuss how the dynamics of such collisional decoherence phenomena are described by the quantum linear Boltzmann equation. We start with the case of pure collisional decoherence, which is present even in the limit of an infinitely massive particle, where no dissipation takes place. We also provide the quantitative connection to recent interference experiments [34], where decoherence due to a background gas was observed as a loss of visibility in the quantum interference fringes. After that, we discuss the related topics of the loss of coherence in case of momentum superpositions and in the Brownian motion limit.

\subsection{The limit of a massive tracer particle}

The incoherent part of the quantum linear Boltzmann equation (2.2) simplifies if the test particle is much more massive than the gas particles. The function $L$ from Eq. (2.3), which provides the details of the collisional interaction, is then replaced by 


$$
L(\mathbf{p}, \mathbf{P} ; \mathbf{Q}) \stackrel{M \gg m}{\rightrightarrows} \sqrt{\frac{n_{\text {gas }}}{m Q}} f\left(\mathbf{p}_{\perp \mathbf{Q}}-\frac{\mathbf{Q}}{2}, \mathbf{p}_{\perp \mathbf{Q}}+\frac{\mathbf{Q}}{2}\right) \sqrt{\mu\left(\mathbf{p}_{\perp \mathbf{Q}}+\frac{\mathbf{Q}}{2}\right)} .
$$

Hence, in this limit the Lindblad operators no longer depend on the test particle momentum P. One can now perform the $\mathrm{d}_{\perp}$-integration in Eq. (2.2), such that, recalling Eq. (2.8) and Eq. (2.9), one gets the much simpler expression

$$
\mathcal{L} \rho=-\int \mathrm{d} \mathbf{Q} M_{\mathrm{in}}^{\mathrm{cl}}(\mathbf{Q} ; \mathbf{Q})\left[\rho-\mathrm{e}^{i \mathbf{Q} \cdot \mathbf{X} / \hbar} \rho \mathrm{e}^{-i \mathbf{Q} \cdot \mathbf{X} / \hbar}\right] .
$$

Here the classical rate $M_{\mathrm{in}}^{\mathrm{cl}}(\mathbf{Q} ; \mathbf{Q})$ must be evaluated with $m_{*} \rightarrow m$. It gives the rate for a particle with vanishing momentum $\mathbf{P}=0$ to experience a momentum change of $\mathbf{Q}$.

It is now helpful to introduce the probability distribution of the possible momentum transfers according to

$$
\mathcal{P}(\mathbf{Q})=\frac{M_{\mathrm{in}}^{\mathrm{cl}}(\mathbf{Q} ; \mathbf{Q})}{\int \mathrm{d} \mathbf{Q}^{\prime} M_{\mathrm{in}}^{\mathrm{cl}}\left(\mathbf{Q}^{\prime} ; \mathbf{Q}^{\prime}\right)}
$$

The normalization, i.e., the total rate of collisions is given by the classical loss rate known from Eq. (2.12),

$$
\Gamma_{\text {tot }} \equiv M_{\text {out }}^{\mathrm{cl}}(0)=\int \mathrm{d} \mathbf{Q}^{\prime} M_{\mathrm{in}}^{\mathrm{cl}}\left(\mathbf{Q}^{\prime} ; \mathbf{Q}^{\prime}\right)
$$

It is determined by the total scattering cross-section and therefore always finite.

We can thus write Eq. (7.2) in the compact form [48]

$$
\mathcal{L} \rho=-\Gamma_{\text {tot }} \int \mathrm{d} \mathbf{Q} \mathcal{P}(\mathbf{Q})\left(\rho-\mathrm{e}^{i \mathbf{Q} \cdot \mathbf{X} / \hbar} \rho \mathrm{e}^{-i \mathbf{Q} \cdot \mathbf{X} / \hbar}\right) .
$$

It puts into evidence that the incoherent dynamics is simply given by momentum kicks

$$
\rho \rightarrow \mathrm{e}^{i \mathbf{Q} \cdot \mathbf{X} / \hbar} \rho \mathrm{e}^{-i \mathbf{Q} \cdot \mathbf{X} / \hbar},
$$

characterized by a random momentum transfer $\mathbf{Q}$, which is distributed according to Eq. (7.3). As discussed below, Eq. (7.5) induces an exponential decay of the off-diagonal elements in position representation, i.e., a "localization" of a spatial superposition into a statistical mixture.

A useful explicit expression is obtained from Eq. (7.2) by introducing the variables $\mathbf{p}_{i} \equiv p \mathbf{n}_{i}=\mathbf{p}+\mathbf{Q} / 2$ and $\mathbf{p}_{f} \equiv p \mathbf{n}_{f}=\mathbf{p}-\mathbf{Q} / 2$,

$$
\mathcal{L} \rho=-n_{\text {gas }} \int_{0}^{\infty} \mathrm{d} p \bar{\nu}(p) \frac{p}{m} \int \frac{\mathrm{d} \Omega_{\mathbf{n}_{i}}}{4 \pi} \int \mathrm{d} \Omega_{\mathbf{n}_{f}}\left|f\left(p \mathbf{n}_{f}, p \mathbf{n}_{i}\right)\right|^{2}\left[\rho-\mathrm{e}^{i\left(\mathbf{p}_{i}-\mathbf{p}_{f}\right) \cdot \mathbf{X} / \hbar} \rho \mathrm{e}^{-i\left(\mathbf{p}_{i}-\mathbf{p}_{f}\right) \cdot \mathbf{X} / \hbar}\right],
$$

where $\bar{\nu}(p)=4 \pi p^{2} \mu_{\beta}(p \mathbf{n})$ is the Maxwell distribution of the momentum magnitudes in the gas.

Equation (7.7) corresponds to the result of Gallis and Fleming [43, once amended for an incorrect factor of $2 \pi$. This little flaw, which is however experimentally relevant, was not due to a simple calculational error, but goes back to an incorrect treatment of a "square of $\delta$-functions" in [40. Such a strictly ill-defined expression arises if asymptotic scattering theory is naively incorporated into a dynamic description. One can fix the factor either by circumventing the S-matrix in a weak-coupling treatment 46 150154/49/77. or by formally modifying the S-matrix such that asymptotically outgoing states are not transformed [54]66[32.

The equation of pure collisional decoherence discussed so far was obtained by the naive limit $M \rightarrow \infty$ of the quantum linear Boltzmann equation. As a result, the incoherent part Eq. (2.2) depends only on the position operator $\mathrm{X}$ of the test particle. Also in the coherent part of Eq. (2.1) the dependence on $\mathrm{P}$ gets lost in this limit, since the kinetic energy $\mathrm{P}^{2} /(2 M)$ vanishes, while the gas induced energy shift $H_{\mathrm{n}}(\mathrm{P})$ turns into an (unobservable) constant. It follows that the dynamics of the momentum distribution of the test particle state remains frozen under Eq. (7.7). 
The full quantum linear Boltzmann equation provides of course a much more refined description of the dynamics. However, for a massive test particle and at short time scales, where the effects of an energy exchange between particle and gas are negligible, an improved version of Eq. (7.2) may already be sufficient in many situations. A natural improvement is suggested by the fact that the loss term (7.4) is evaluated for a test particle at rest.

Provided that the test particle is very massive, such that its momentum varies slowly on the short time scale of decoherence, it may be allowed to replace the momentum operator in Eq. (2.2) by its initial expectation value $\mathbf{P}_{0}=\langle\mathrm{P}\rangle_{\rho_{0}}$. This leads to an equation very similar to Eq. (17.2),

$$
\mathcal{L} \rho=-\int \mathrm{d} \mathbf{Q} M_{\text {in }}^{\mathrm{cl}}\left(\mathbf{P}_{0}+\mathbf{Q} ; \mathbf{Q}\right)\left[\rho-\mathrm{e}^{i \mathbf{Q} \cdot \mathbf{X} / \hbar} \rho \mathrm{e}^{-i \mathbf{Q} \cdot \mathbf{X} / \hbar}\right] .
$$

Introducing the normalized probability density

$$
\mathcal{P}_{\mathbf{P}_{0}}(\mathbf{Q})=\frac{M_{\mathrm{in}}^{\mathrm{cl}}\left(\mathbf{P}_{0}+\mathbf{Q} ; \mathbf{Q}\right)}{\int \mathrm{d} \mathbf{Q}^{\prime} M_{\mathrm{in}}^{\mathrm{cl}}\left(\mathbf{P}_{0}+\mathbf{Q}^{\prime} ; \mathbf{Q}^{\prime}\right)},
$$

one arrives at

$$
\mathcal{L} \rho=-\Gamma_{\text {tot }}\left(\mathbf{P}_{0}\right) \int \mathrm{d} \mathbf{Q} \mathcal{P}_{\mathbf{P}_{0}}(\mathbf{Q})\left[\rho-\mathrm{e}^{i \mathbf{Q} \cdot \mathbf{X} / \hbar} \rho \mathrm{e}^{-i \mathbf{Q} \cdot \mathbf{X} / \hbar}\right]
$$

where the rate $\Gamma_{\text {tot }}\left(\mathbf{P}_{0}\right)=M_{\text {out }}^{\text {cl }}\left(\mathbf{P}_{0}\right)$ gives the classical rate of collisions experienced by a test particle with momentum $\mathbf{P}_{0}$.

The difference with respect to Eq. (7.5) lies in the residual, parametric dependence on the momentum of the test particle $\mathbf{P}_{0}$, leading to a more realistic estimate for the total scattering rate $\Gamma_{\text {tot }}\left(\mathbf{P}_{0}\right)$ and thus of collisional decoherence. This is of relevance for the quantitative description of experiments on gas-induced decoherence 3955151106.

It is clear that Eq. (7.10) cannot account for the long time dynamics of momentum and kinetic energy, since the momentum of the test particle here only appears as a classical label. As far as the short time dynamics is concerned, we note that the corresponding random momentum transfers (7.6) are here incorporated in full, without the assumption $|Q \Delta X / \hbar| \ll 1$, used in Sect. 5 when considering the limit of quantum Brownian motion.

\subsection{Decoherence in position}

\subsubsection{The exact solution of pure decoherence}

In position representation Eq. (7.5) takes the simple form

$$
\left\langle\mathbf{X}|\mathcal{L} \rho| \mathbf{X}^{\prime}\right\rangle=-\Gamma_{\text {tot }}\left[1-\Phi_{\mathcal{P}}\left(\mathbf{X}-\mathbf{X}^{\prime}\right)\right]\left\langle\mathbf{X}|\rho| \mathbf{X}^{\prime}\right\rangle,
$$

where $\Phi_{\mathcal{P}}(\mathbf{X})$ denotes the Fourier transform of the probability density (7.3) of momentum transfers, i.e., its characteristic function 152

$$
\Phi_{\mathcal{P}}(\mathbf{S})=\int \mathrm{d} \mathbf{Q} \mathcal{P}(\mathbf{Q}) \mathrm{e}^{i \mathbf{Q} \cdot \mathbf{S} / \hbar}
$$

The solution of the master equation $\mathrm{d} \rho / \mathrm{d} t=\mathcal{L} \rho$ then shows an exponential decay of the position off-diagonal elements,

$$
\left\langle\mathbf{X}\left|\rho_{t}\right| \mathbf{X}^{\prime}\right\rangle=\mathrm{e}^{-\Gamma_{\text {tot }}\left[1-\Phi_{\mathcal{P}}\left(\mathbf{X}-\mathbf{X}^{\prime}\right)\right] t}\left\langle\mathbf{X}\left|\rho_{0}\right| \mathbf{X}^{\prime}\right\rangle
$$

The position density is not affected, $\left\langle\mathbf{X}\left|\rho_{t}\right| \mathbf{X}\right\rangle=\left\langle\mathbf{X}\left|\rho_{0}\right| \mathbf{X}\right\rangle$, since $\Phi_{\mathcal{P}}(0)=1$.

The function $\Phi_{\mathcal{P}}\left(\mathbf{X}-\mathbf{X}^{\prime}\right)$ describes the effect of a single collision event on the statistical operator in the position representation, $\left\langle\mathbf{X}|\rho| \mathbf{X}^{\prime}\right\rangle \rightarrow \Phi_{\mathcal{P}}\left(\mathbf{X}-\mathbf{X}^{\prime}\right)\left\langle\mathbf{X}|\rho| \mathbf{X}^{\prime}\right\rangle$. It is sometimes called the "decoherence 
function" since it amounts to the overlap of two scattered gas particle states displaced by $\mathbf{X}-\mathbf{X}^{\prime}$, which quantifies to what extent the gas can distinguish between the two positions.

It follows from Eq. (7.3) that in terms of the scattering amplitude it is explicitly given by

$$
\Phi_{\mathcal{P}}(\mathbf{S})=\frac{n_{\text {gas }}}{\Gamma_{\text {tot }}} \int \mathrm{d} p \bar{\nu}(p) \frac{p}{m} \int \frac{\mathrm{d} \Omega_{\mathbf{n}_{i}}}{4 \pi} \int \mathrm{d} \Omega_{\mathbf{n}_{f}}\left|f\left(p \mathbf{n}_{f}, p \mathbf{n}_{i}\right)\right|^{2} \mathrm{e}^{i p\left(\mathbf{n}_{i}-\mathbf{n}_{f}\right) \cdot \mathbf{S} / \hbar} .
$$

In the case of isotropic scattering, the decoherence effect depends only on the distance $S=\left|\mathbf{X}-\mathbf{X}^{\prime}\right|$ of the positions considered. It then takes the form

$$
\Phi_{\mathcal{P}}(S)=2 \pi \frac{n_{\text {gas }}}{\Gamma_{\text {tot }}} \int \mathrm{d} p \bar{\nu}(p) \frac{p}{m} \int_{-1}^{1} \mathrm{~d}(\cos \theta)\left|f\left(\cos \theta ; \frac{p^{2}}{2 m}\right)\right|^{2} \operatorname{sinc}\left(2 p \sin \left(\frac{\theta}{2}\right) \frac{S}{\hbar}\right),
$$

with $\theta$ the scattering angle and $\operatorname{sinc}(x)=\sin (x) / x$. Due to the infinite test mass the momentum transfer is given by the expression $Q=2 p \sin (\theta / 2)$, which appears in the argument of the sinc-function. The effect of this function in Eq. (7.15) is thus to suppress position coherences the more the better the scattered state with its resolving capacity $\hbar / Q$ can distinguish between two possible scattering positions with distance $S$.

The second ingredient to Eq. (7.13) is the total rate of collisions $\Gamma_{\text {tot }}$ defined in Eq. (7.4). The effect and the rate of collisions are thus fixed by $\Phi_{\mathcal{P}}$ and $\Gamma_{\text {tot }}$ separately, which can be of great importance in physical applications. We note in particular that the function

$$
\Psi(\mathbf{S}, t)=\mathrm{e}^{-\Gamma_{\text {tot }}\left[1-\Phi_{\mathcal{P}}(\mathbf{S})\right] t}
$$

appearing in Eq. (7.13) is itself a characteristic function, namely that of a compound Poisson process 153 152. Such a process differs from a simple Poisson process in that the jump events, here the collisions, do not have a fixed size, while the waiting time distribution between the events is Poissonian. Rather, the jump size is itself a random variable, here the momentum transfer in a single collision, which is distributed according to $\mathcal{P}(\mathbf{Q})$.

This physical picture behind the collisional dynamics is best seen by expanding the exponential in Eq. (7.13), which corresponds to a jump expansion of the solution of the master equation

$$
\left\langle\mathbf{X}\left|\rho_{t}\right| \mathbf{X}^{\prime}\right\rangle=\sum_{n=0}^{\infty} p_{n}(t) \Phi_{\mathcal{P}}^{n}\left(\mathbf{X}-\mathbf{X}^{\prime}\right)\left\langle\mathbf{X}\left|\rho_{0}\right| \mathbf{X}^{\prime}\right\rangle
$$

Here $p_{n}(t)=\mathrm{e}^{-\Gamma_{\text {tot }} t}\left(\Gamma_{\text {tot }} t\right)^{n} / n$ ! is the probability to have $n$ collisions during the time $t$, given by the Poisson distribution with parameter $\Gamma_{\text {tot }}$. The associated effect is the multiplication of the initial statistical operator in the position representation with the $n$-th power of the decoherence function $\Phi_{\mathcal{P}}$ for the effect of a single collision.

Equation (7.13) is a particular case of a more general class of solutions of master equations for the description of decoherence, applying in the presence of translational invariance. In the general case, the characteristic function $\Psi(\mathbf{X}, t)$ corresponding to Eq. (7.16) is the characteristic function of a Lévy process, of which compound Poisson processes are a particular example 29|152. This result can be obtained considering the general structure of translation-covariant Markovian master equations obtained by Holevo, and considering its limiting expression relevant for the description of decoherence. This provides a common theoretical framework for the description of quite different decoherence experiments [154]. The solution of such master equations reads

$$
\left\langle\mathbf{X}\left|\rho_{t}\right| \mathbf{X}^{\prime}\right\rangle=\Psi\left(\mathbf{X}-\mathbf{X}^{\prime}, t\right)\left\langle\mathbf{X}\left|\rho_{0}\right| \mathbf{X}^{\prime}\right\rangle,
$$

and due to the general properties of characteristic functions it naturally describes decoherence effects. In fact, it is a defining property of characteristic functions that $\Psi(0, t)=1$, while $\left|\Psi\left(\mathbf{X}-\mathbf{X}^{\prime}, t\right)\right| \leqslant 1$, such that diagonal matrix elements are not affected, while off-diagonal matrix elements are generally suppressed. In particular, for the characteristic function of a Lévy process one has $\lim _{t \rightarrow \infty} \Psi\left(\mathbf{X}-\mathbf{X}^{\prime}, t\right)=0$ for $\mathbf{X} \neq \mathbf{X}^{\prime}$ [153, such that all coherences are completely lost with elapsing time. For fixed interaction time $t$, on the 
other hand, a variety of position dependencies are possible, from an exponential loss of coherence to revivals ending up with a constant decoherence rate. All such behaviors are comprised in the general expression of the characteristic function of a Lévy process, provided by the Lévy-Khintchine formula, one of the beautiful results of probability theory 152 .

\subsubsection{Experimental tests}

One of the most important experimental verifications of the quantum linear Boltzmann equation comes from its application to the quantitative explanation of collisional decoherence observed in near-field interferometry experiments with large molecules 39 55. In such experiments a velocity selected molecular beam from a thermal source passes an interferometer made up of material gratings. After flooding the vacuum chamber with various background gases one records the reduction of visibility of the interference fringes as a function of the gas pressure. This decrease of the interference contrast is directly related to the loss of coherence in the position off-diagonal elements in the state of motion, which was discussed in the previous section.

An important requirement for the experimental observation of collisional decoherence is that the interfering molecule is not removed from the interferometer by the interaction with the background gas. As described in Sect. 6, such a loss process would lead to a reduction of the count rate, but it would not diminish the interference contrast of the detected molecules. This is the reason why relatively heavy $\mathrm{C}_{70}$ fullerene molecules were required to observe the effect in 3955, while the Mach-Zehnder-type atom interferometers realized so far cannot observe collisional decoherence with room temperature gases. We note that the loss of visibility in a sodium atom interferometer reported in 155 is therefore due to a different mechanism, namely the gas-induced noise at the preparation, i.e., the slit collimation and at the detection stage of the interferometer beam. The atomic motion between preparation and detection is there coherent, in contrast, such that this gas-induced effect is not described by the theory of Sect. 7.2.1.

Due to the large mass of the interfering particles and the short time of flight in [3955], one is allowed to neglect relaxation effects in the quantum description of the interfering test particle. The limit of Sect. 7.1 thus applies, and one expects in particular that the dynamics is correctly described by Eq. (7.10), which accounts for the finite velocity of the molecular beam with respect to the thermal gas. In the position representation the equation reads as

$$
\left\langle\mathbf{X}|\mathcal{L} \rho| \mathbf{X}^{\prime}\right\rangle=-\Gamma_{\text {tot }}\left(\mathbf{P}_{0}\right)\left[1-\Phi_{\mathcal{P}_{\mathbf{P}_{0}}}\left(\mathbf{X}-\mathbf{X}^{\prime}\right)\right]\left\langle\mathbf{X}|\rho| \mathbf{X}^{\prime}\right\rangle
$$

where $\mathbf{P}_{0}$ is now the mean momentum of the incoming fullerenes. It determines the rate $\Gamma_{\text {tot }}\left(\mathbf{P}_{0}\right)=$ $M_{\text {out }}^{\text {cl }}\left(\mathbf{P}_{0}\right)$, as well as the decoherence function

$$
\Phi_{\mathcal{P}_{\mathbf{P}_{0}}}(\mathbf{X})=\int \mathrm{d} \mathbf{Q} M_{\mathrm{in}}^{\mathrm{cl}}\left(\mathbf{P}_{0}+\mathbf{Q} ; \mathbf{Q}\right) \mathrm{e}^{i \mathbf{Q} \cdot \mathbf{X} / \hbar}
$$

The classical rate $M_{\mathrm{in}}^{\mathrm{cl}}$, defined in Eq. (2.10), includes the differential scattering cross-section for collisions between fullerene and gas molecules.

The interaction between the gas molecules and the fullerenes is well described in the experiment by the isotropic part of the London dispersion force. It corresponds to a potential of the form $U(r)=-C_{6} / r^{6}$, with an interaction strength $C_{6}$, which can be calculated for different gases [156 39]. The associated total scattering cross-section can be obtained in a semiclassical calculation [157]; it depends on the relative velocity as

$$
\sigma_{\text {tot }}(\mathbf{v}-\mathbf{V})=\frac{\pi^{2}}{\sin (\pi / 5) \Gamma(2 / 5)}\left(\frac{3 \pi C_{6}}{8 \hbar}\right)^{2 / 5}|\mathbf{v}-\mathbf{V}|^{-\frac{2}{5}}
$$

A quantitative description of the general loss of coherence in a near field interferometer due to master equations of the form (7.10) was presented in [151. However, the situation is somewhat simpler in the experiment described in 3955] since the background gases are at room temperature. This implies that for practically all positions in the interferometer the collisions are characterized by large momentum transfers 
$Q$ in the sense that $Q \gg \hbar / S$, if $S$ denotes the separation of the interference paths. As follows from Eq. (7.15), already a single collision then suffices to completely reduce the off-diagonal elements of the position representation, $\Phi_{\mathcal{P}}(S \neq 0) \simeq 0$, while $\Phi_{\mathcal{P}}(0)=1$. Only those molecules then contribute to the contrast of the interference pattern which experienced no collisions, while the others contribute only to the unstructured background.

In this situation the loss of visibility is determined only by the collision rate $\Gamma_{\text {tot }}\left(\mathbf{P}_{0}\right)$ in Eq. (7.19), determined by the loss term $\Gamma_{\text {tot }}\left(\mathbf{P}_{0}\right)=M_{\text {out }}^{\text {cl }}\left(\mathbf{P}_{0}\right)$ in the linear Boltzmann equation [106]79, according to the formula 39

$$
\mathcal{V}=\mathcal{V}_{0} \mathrm{e}^{-\Gamma_{\text {tot }}\left(\mathbf{P}_{0}\right) t}
$$

Here $\mathcal{V}_{0}$ is the visibility in the absence of the gas and $t$ is the time of flight of the fullerenes crossing the interferometer.

It is therefore sufficient to evaluate the collision frequency $\Gamma_{\text {tot }}\left(\mathbf{P}_{0}\right)$, which is best described by an effective scattering cross-section

$$
\Gamma_{\text {tot }}\left(\mathbf{P}_{0}\right)=n_{\text {gas }} \frac{P_{0}}{M} \sigma_{\text {eff }}\left(P_{0}\right) .
$$

The expression $\sigma_{\text {eff }}\left(P_{0}\right)$ for a total cross-section of the form Eq. (7.20) has already been presented in Sect. 4.3.3. According to Eq. (4.47), evaluated for $a=-2 / 5$, we have

$$
\Gamma_{\text {tot }}\left(\mathbf{P}_{0}\right)=n_{\text {gas }} \frac{4 \pi \Gamma(9 / 10)}{5 \sin (\pi / 5)}\left(\frac{3 \pi C_{6}}{2 \hbar}\right)^{2 / 5} v_{\beta}^{\frac{3}{5}}{ }_{1} F_{1}\left(-\frac{3}{10}, \frac{3}{2} ;-\left(\frac{P_{0}}{M v_{\beta}}\right)^{2}\right) .
$$

In the experiment, the beam velocity is small compared to the thermal velocities of the gas, $P_{0} /\left(M v_{\beta}\right) \ll 1$. The expression can then be approximated as [39/55/106]

$$
\Gamma_{\text {tot }}\left(\mathbf{P}_{0}\right)=n_{\text {gas }} \frac{4 \pi \Gamma(9 / 10)}{5 \sin (\pi / 5)}\left(\frac{3 \pi C_{6}}{2 \hbar}\right)^{2 / 5} v_{\beta}^{\frac{3}{5}}\left[1+\frac{1}{5}\left(\frac{P_{0}}{M v_{\beta}}\right)^{2}+\mathcal{O}\left(\left(\frac{P_{0}}{M v_{\beta}}\right)^{4}\right)\right] .
$$

One can introduce a critical gas pressure for the onset of decoherence in terms of the effective scattering cross-section introduced in Eq. (7.22),

$$
p_{0}=\frac{M k_{\mathrm{B}} T}{P_{0} \sigma_{\mathrm{eff}}\left(P_{0}\right) t}
$$

One therefore expects an exponential loss of visibility of the interference fringes with growing gas pressure according to

$$
\mathcal{V}=\mathcal{V}_{0} \mathrm{e}^{-p / p_{0}} .
$$

This behavior was observed in the experiments [39/55], in quantitative agreement with theory, see Fig. 7.1

Finally, we mention that the reduction of interference contrast due to momentum exchange effects was the subject of several other experimental [158|159|160|161|162|163] and theoretical [151|164|154|165|166|167] investigations. While the physical mechanism is here the scattering, absorption, or emission of photons, the dynamic effect on the motional state of the interfering particle can still be described with a master equation of the form (7.5), (7.11). All that needs to be done is to adjust the rate of events $\Gamma_{\text {tot }}$ according to the physical mechanism considered, as well as the decoherence function $\Phi_{\mathcal{P}}$ for the effect of a single event [154]. Studies of other environmental decoherence effects in interferometers were presented in [168[169 170]171. These are based on master equations characteristic of linear coupling models, which are of the form of the Brownian motion master equation discussed in Sect. 7.4 


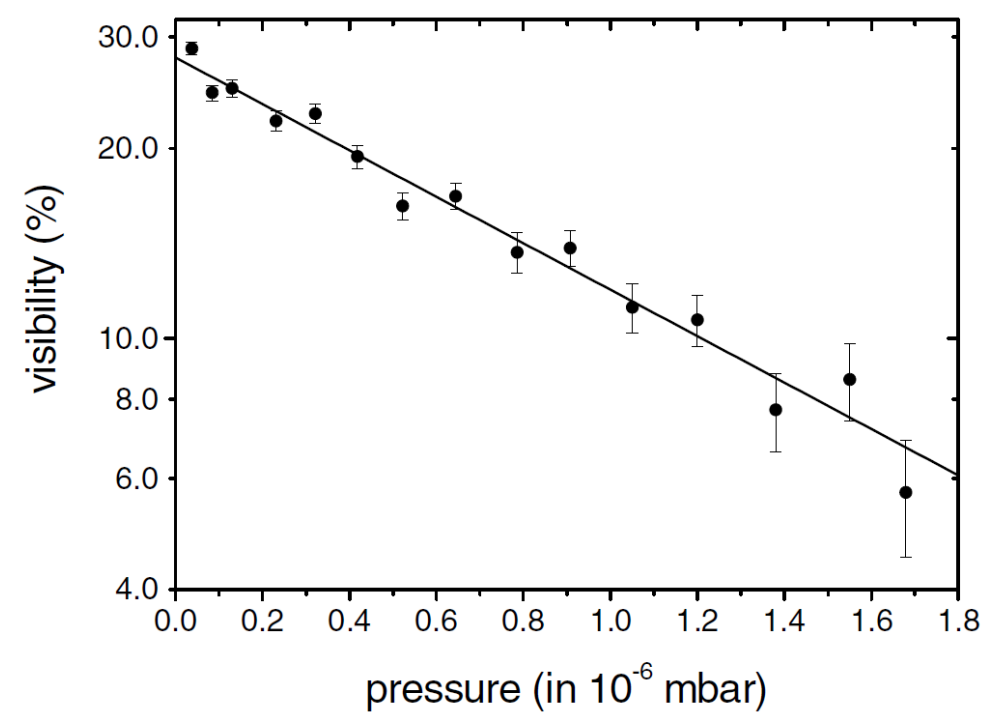

Fig. 7.1. Experimentally observed visibility of the interference fringes of $\mathrm{C}_{70}$ fullerenes in an argon gas at room temperature, as a function of the gas pressure (dots). The solid line gives the prediction of decoherence theory. It is based on Eq. (7.26), but also accounts for gas-induced modifications of the particular gravitational velocity selection scheme employed in the experiment. (Figure taken from [55])

\subsection{Decoherence in momentum}

As described in the previous section, it is relatively simple to evaluate and to experimentally test the decay of coherence in superpositions of position eigenstates. The analogous case of superpositions of momentum eigenstates is more involved. On the theoretical side, the momentum operator of the test particle can no longer be considered a classical label in the limiting expression of the quantum linear Boltzmann equation, but one deals with a master equation for the operators $\mathrm{X}$ and $\mathrm{P}$ of the test particle. From the experimental point of view, it is difficult to conceive experiments testing decoherence in momentum space. The source must generate a state showing longitudinal momentum coherences, which requires a non-stationary beam preparation, as well as an interferometric setup permitting to monitor the presence of such coherences 172 173. This is complicated by the fact that the decoherence in position discussed in Sect. 7.2 is always present and may quickly dominate. It affects superposition states of different momenta, because the free evolution of such states unavoidably leads to a superposition of spatially separated wave packets.

In order to study the decoherence dynamics of momentum superpositions we exploit the quantum trajectory algorithm presented in Sect. 4.3.2. taking as initial condition a coherent superposition of momentum eigenstates. We start with an initial state of the form

$$
|\psi(0)\rangle=\sum_{i=1}^{N} \alpha_{i}(0)\left|\mathbf{U}_{i}(0)\right\rangle,
$$

where the complex amplitudes $\alpha_{i}$ satisfy the normalization condition $\sum_{i=1}^{N}\left|\alpha_{i}(0)\right|^{2}=1$. As discussed in Sect. 4.3.2, the state at a later time $t$ will still be in a superposition of $N$ momentum eigenstates, but with different amplitudes $\alpha_{i}(t)$ and scaled momenta $\mathbf{U}_{i}(t)$

$$
|\psi(t)\rangle=\sum_{i=1}^{N} \alpha_{i}(t)\left|\mathbf{U}_{i}(t)\right\rangle .
$$

Given a jump into the state Eq. (7.28) occurring at time $t$, the deterministic time evolution described by Eq. (4.39) only modifies the amplitudes, 


$$
\alpha_{i}(t+\tau)=\frac{\mathrm{e}^{-i E\left(U_{i}(t)\right) \tau / \hbar} \mathrm{e}^{-\tilde{\Gamma}\left(U_{i}(t)\right) \tau / 2}}{\sqrt{\sum_{j=1}^{N}\left|\alpha_{j}(t)\right|^{2} \mathrm{e}^{-\tilde{\Gamma}\left(U_{j}(t)\right) \tau}}} \alpha_{i}(t),
$$

with $E(U)=M v_{\beta}^{2} U^{2} / 2$ the kinetic energy. The cumulative distribution function $F(\tau)$ of Eq. (4.40) for the waiting time until the next jump is now given by a sum of exponential functions,

$$
F(\tau)=1-\sum_{j=1}^{N}\left|\alpha_{j}(t)\right|^{2} \mathrm{e}^{-\tilde{\Gamma}\left(U_{j}(t)\right) \tau} .
$$

The jump modifies amplitudes and momenta as

$$
\begin{aligned}
\mathbf{U}_{i}(t) & \rightarrow \mathbf{U}_{i}(t)+\frac{m_{*}}{M} \mathbf{K} \\
\alpha_{i}(t+\tau) & \rightarrow f_{i} \alpha_{i}(t+\tau)
\end{aligned}
$$

with

$$
f_{i}=\frac{\mathrm{e}^{-\left(K / 2+\mathbf{K} \cdot \mathbf{U}_{i}(t)\right)^{2} / 2}}{\sqrt{\sum_{j=1}^{N}\left|\alpha_{j}(t)\right|^{2} \mathrm{e}^{-\left(K / 2+\mathbf{K} \cdot \mathbf{U}_{j}(t)\right)^{2}}}} .
$$

While all momentum eigenstates in the superposition are shifted by the same amount, their different amplitudes are modified in different ways. The momentum transfer $\mathbf{K}$ characterizing the jump is distributed according to Eq. (4.42) with the state $|\psi(t)\rangle$ given by Eq. (7.28). The probability density Eq. (4.55) is therefore replaced by the mixture

$$
\widetilde{\mathcal{P}}(K, \xi)=\sum_{i=1}^{N} \lambda_{i} \mathcal{P}\left(K, \xi_{i}\right),
$$

where the weights $\lambda_{i}$ are given by

$$
\lambda_{i}=\frac{\left|\alpha_{i}(t)\right|^{2} \mathrm{e}^{-\tilde{\Gamma}\left(U_{i}(t)\right) \tau} \tilde{\Gamma}\left(U_{i}(t)\right)}{\sum_{j=1}^{N}\left|\alpha_{j}(t)\right|^{2} \mathrm{e}^{-\tilde{\Gamma}\left(U_{j}(t)\right) \tau} \tilde{\Gamma}\left(U_{j}(t)\right)} .
$$

The probability density $\mathcal{P}(K, \xi)$ is defined in Eq. (4.55) and $\xi_{i}=\mathbf{U}_{i} \cdot \mathbf{K} /\left(U_{i} K\right)$ is the cosine of the angle between $\mathbf{K}$ and $\mathbf{U}_{i}(t)$.

We now specialize to a superposition of two momentum eigenstates with equal amplitudes $\alpha_{1}(0)=$ $\alpha_{2}(0)=1 / \sqrt{2}$,

$$
|\psi(0)\rangle=\frac{1}{\sqrt{2}}\left(\left|\mathbf{U}_{1}(0)\right\rangle+\left|\mathbf{U}_{2}(0)\right\rangle\right),
$$

and evaluate the temporal behavior of the matrix elements in position representation. The off-diagonal elements provide a measure for the coherence in the state of motion

$$
C(t)=\mathbb{E}\left[\left|\frac{\alpha_{1}(t) \alpha_{2}^{*}(t)}{\alpha_{1}(0) \alpha_{2}^{*}(0)}\right|\right] .
$$

This quantity is plotted in Fig. 7.2 for the choice $\mathbf{U}_{1}(0)=-\mathbf{U}_{2}(0)=\mathbf{U}_{0}$, such that the distance in momentum space is given by $\left|\mathbf{U}_{1}(0)-\mathbf{U}_{2}(0)\right|=2 U_{0}$. One observes an exponential decay in time in analogy to the behavior (7.13) of the position coherences.

Based on this observation we now assume that the decay has the generic form

$$
C(t)=\mathrm{e}^{-\Lambda\left(U_{0}\right) t} .
$$

We proceed to evaluate how the decay constant $\Lambda$ depends on the value $U_{0}$ characterizing the initial momentum separation and to compare the analytic result with the simulations. 


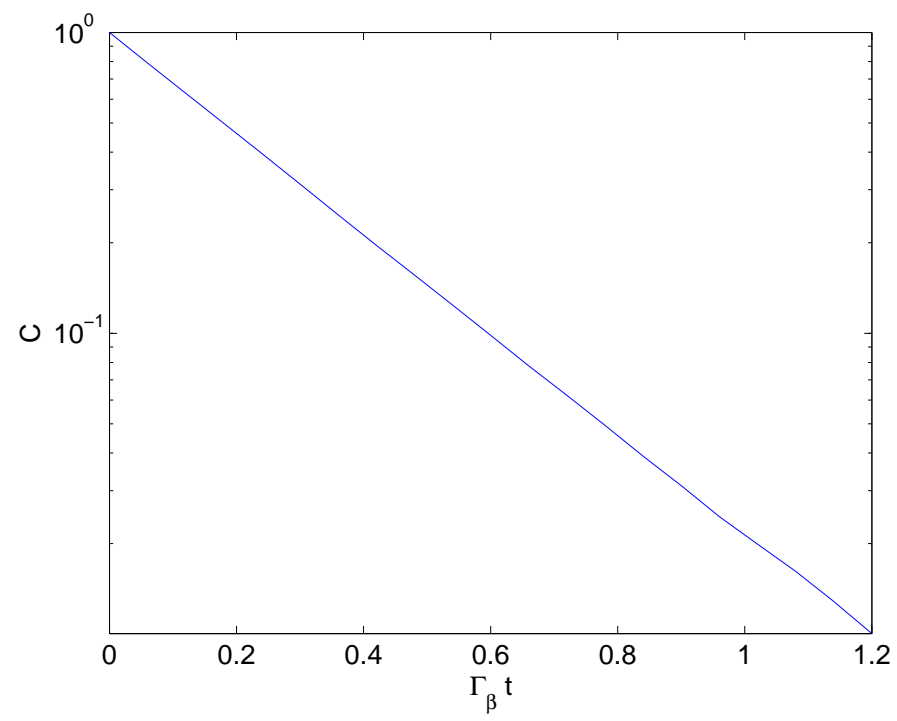

Fig. 7.2. Semilogarithmic plot of the coherence $C(t)$ of a superposition of momentum eigenstates, as defined in Eqs. (7.34), 17.35. One observes a clear exponential decay, in analogy to the behavior of position superpositions. The plot corresponds to a constant cross-section with $U_{0}=4$ and a mass ratio of $m / M=1$; the time is given in units of the inverse total scattering rate 4.28).

The quantum trajectory method presented in Sect. 4.3 .2 is equivalent to solving the master equation. One can thus assess the decay constant by noting that for short times $C(t)$ either does not change, with a probability $1-\tilde{\Gamma}\left(U_{0}\right) t$, or it becomes $f_{1} f_{2}$ if a jump takes place. Neglecting higher order jump processes one thus has

$$
C(t) \approx 1-\Lambda\left(U_{0}\right) t \approx 1-\tilde{\Gamma}\left(U_{0}\right) t+\tilde{\Gamma}\left(U_{0}\right) t\left\langle f_{1} f_{2}\right\rangle,
$$

where $\langle\cdots\rangle$ denotes the average over the different possible momentum transfers. Exploiting the expression Eq. (7.32) and averaging with respect to Eq. (7.33) one comes to

$$
\Lambda\left(U_{0}\right) \approx \tilde{\Gamma}\left(U_{0}\right)\left(1-\left\langle f_{1} f_{2}\right\rangle\right) .
$$

For the case of a constant scattering cross-section, it takes the explicit form

$$
\Lambda\left(U_{0}\right)=\tilde{\Gamma}\left(U_{0}\right)-\Gamma_{\beta} \frac{\operatorname{erf}\left(U_{0}\right)}{U_{0}},
$$

with $\Gamma_{\beta}$ defined in Eq. (4.28). As shown in Fig. 7.3 this prediction is in very good agreement with the values of $\Lambda\left(U_{0}\right)$ extracted from the numerical simulations for various initial values of the momentum $U_{0}$. Similar results can be obtained for different scattering cross-sections, the degree of accuracy of the analytic formula depending on the considered scattering cross-section.

A direct comparison of relaxation and decoherence rate in momentum space can be made for the case $m / M \ll 1$ and assuming that the momentum separation is large compared to the thermal momentum of the gas $U_{0} \gg 1$. The relaxation rate is then given by $\eta$ as in Eq. (4.33), while the decoherence rate Eq. (7.39) can be approximated for $U_{0} \gg 1$ by $\Lambda\left(U_{0}\right) \approx \Gamma_{\beta} U_{0}$. The ratio of relaxation and decoherence rate

$$
\frac{\Lambda}{\eta} \approx \frac{3 \sqrt{\pi}}{8} \frac{M}{m} U_{0} \gg 1
$$

indicates that momentum decoherence takes place on a much shorter time scale than relaxation effects.

The study of how decoherence takes place in momentum space is relevant also for the description of the transition from the quantum linear Boltzmann equation to the classical linear Boltzmann equation, already 


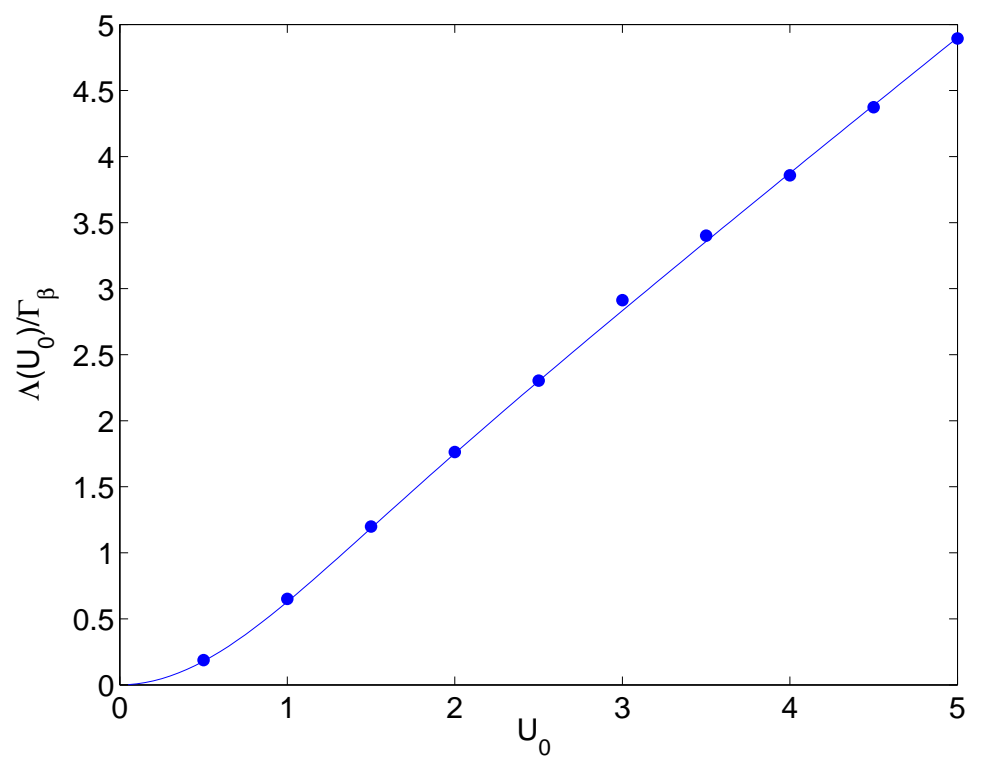

Fig. 7.3. Plot of the decoherence rate $\Lambda$ in units of the collision rate $\Gamma_{\beta}$ as a function of the initial scaled momentum $U_{0}$. The points represent least square fits of the simulated data for the mass $m / M=1$, while the line represents the analytic estimate given by Eq. (7.39).

discussed at the end of Sect. 4.2, As observed in Sect. 2.2, the diagonal matrix elements of the quantum linear Boltzmann equation coincide with the expression of the classical linear Boltzmann equation, provided one considers the quantum scattering cross-section. The dynamical suppression of the off-diagonal matrix elements in the momentum representation as a consequence of decoherence thus amounts to the onset of classical dynamics as far as the momentum observable is concerned.

\subsection{Decoherence in the quantum Brownian motion limit}

Having discussed how the full quantum linear Boltzmann equation can be used to describe decoherence both in position and momentum space, let us now address its description in the Brownian motion limit of the equation. The resulting master equation, which corresponds to approximating the environment by a bath of linearly coupled harmonic oscillators, is most often used to discuss decoherence effects 145/146 147/148149/29. In the simplest case, it leads to an exponential decay rate of the off-diagonal matrix elements in the position representation, which depends like a Gaussian on the position separation. This picture corresponds to the common lore, which however obviously cannot cover all interesting physical situations, neither theoretically [174]175|176]177, nor experimentally [158|159|160|161|39|162].

We start with the master equation obtained by neglecting the friction term in the equation (5.15) of quantum Brownian motion.

$$
\mathcal{L} \rho=-\frac{i}{\hbar}\left[\mathrm{H}_{0}, \rho\right]-\frac{D_{p p}}{\hbar^{2}} \sum_{j=1}^{3}\left[\mathrm{X}_{j},\left[\mathrm{X}_{j}, \rho\right]\right]-\frac{D_{x x}}{\hbar^{2}} \sum_{j=1}^{3}\left[\mathrm{P}_{j},\left[\mathrm{P}_{j}, \rho\right]\right] .
$$

Since the friction term in Eq. (5.15) accounts for energy dissipation, one expects that Eq. (7.41) still describes the short time decoherence phenomena associated with quantum Brownian motion.

\subsubsection{Position representation}

In the position representation, the general solution of Eq. (7.41) can be most easily expressed in terms of the solution of the free Schrödinger equation [118/178/179]. Denoting the freely evolved state at time $t$ as $\rho_{t}^{S}$, we have 


$$
\begin{aligned}
\left\langle\mathbf{X}\left|\rho_{t}\right| \mathbf{X}^{\prime}\right\rangle= & \exp \left(-\frac{D_{p p}}{\hbar^{2}}\left(\mathbf{X}-\mathbf{X}^{\prime}\right)^{2} t\left[1-\frac{\left(D_{p p} / 4 M^{2}\right) t^{2}}{\left[D_{x x}+\left(D_{p p} / 3 M^{2}\right) t^{2}\right]}\right]\right) \\
& \times\left(\frac{1}{4 \pi\left[D_{x x}+\left(D_{p p} / 3 M^{2}\right) t^{2}\right] t}\right)^{3 / 2} \int \mathrm{d} \mathbf{Y} \exp \left(\frac{i}{\hbar} \frac{D_{p p}}{2 M} \frac{\mathbf{Y} \cdot\left(\mathbf{X}-\mathbf{X}^{\prime}\right) t}{\left[D_{x x}+\left(D_{p p} / 3 M^{2}\right) t^{2}\right]}\right) \\
& \times \exp \left(-\frac{Y^{2}}{4\left[D_{x x}+\left(D_{p p} / 3 M^{2}\right) t^{2}\right] t}\right)\left\langle\mathbf{X}-\mathbf{Y}\left|\rho_{t}^{S}\right| \mathbf{X}^{\prime}-\mathbf{Y}\right\rangle .
\end{aligned}
$$

It is easy to recover from Eq. (7.42) the standard result of elementary decoherence models, according to which the loss of coherence in position space is governed by a Gaussian function in the distance $\left|\mathbf{X}-\mathbf{X}^{\prime}\right|$ 18040. In the limit of a very massive particle and short times, the spreading due to the Hamiltonian term can be neglected, $\left(D_{p p} / 3 M^{2}\right) t^{2} \simeq 0$. One is then left with a convolution of the initial statistical operator in position representation with a Gaussian of width $2 D_{x x} t$. On short time scales, and for high temperatures, the Gaussian is strongly peaked. One is then left with the expected expression

$$
\left\langle\mathbf{X}\left|\rho_{t}\right| \mathbf{X}^{\prime}\right\rangle=\exp \left(-\frac{D_{p p}}{\hbar^{2}}\left(\mathbf{X}-\mathbf{X}^{\prime}\right)^{2} t\right)\left\langle\mathbf{X}\left|\rho_{0}\right| \mathbf{X}^{\prime}\right\rangle
$$

\subsubsection{Momentum representation}

In the momentum representation, it is more convenient to directly express the solution with reference to the initial state $\rho_{0}$

$$
\begin{aligned}
\left\langle\mathbf{P}\left|\rho_{t}\right| \mathbf{P}^{\prime}\right\rangle= & \exp \left(-\frac{D_{x x}}{\hbar^{2}}\left(\mathbf{P}-\mathbf{P}^{\prime}\right)^{2} t\right) \exp \left(-\frac{1}{12} \frac{D_{p p}}{\hbar^{2}}\left(\frac{\mathbf{P}-\mathbf{P}^{\prime}}{M} t\right)^{2} t\right) \exp \left(-\frac{i}{\hbar} \frac{P^{2}-P^{\prime 2}}{2 M} t\right) \\
& \times\left(\frac{1}{4 \pi D_{p p} t}\right)^{3 / 2} \int \mathrm{d} \mathbf{Q} \exp \left(-\frac{Q^{2}}{4 D_{p p} t}\right)\left\langle\mathbf{P}-\mathbf{Q}\left|\rho_{0}\right| \mathbf{P}^{\prime}-\mathbf{Q}\right\rangle .
\end{aligned}
$$

Looking at the position matrix elements Eq. (7.42), one notes that the quantum position diffusion term with coefficient $D_{x x}$ mainly corrects the detailed behavior in the exponential suppression of position coherences. In the momentum representation, one has two distinct decoherence mechanisms instead.

The first factor at the right-hand side of Eq. (7.44) is just due to the $\sum_{j=1}^{3}\left[\mathrm{P}_{j},\left[\mathrm{P}_{j}, \rho\right]\right]$ term in Eq. (7.41), in perfect analogy with the $\sum_{j=1}^{3}\left[\mathrm{X}_{j},\left[\mathrm{X}_{j}, \rho\right]\right]$ term causing decoherence in position. The remaining part comes from the joint effect of free evolution and the term causing decoherence in position. As already mentioned, initial superpositions of different momenta lead to position superpositions as time evolves, such that localization effects also destroy coherences in momentum representation. This asymmetry between position and momentum is clearly due to the presence of the kinetic term. Indeed, Eq. (7.42) and Eq. (7.44) acquire the same functional form in the limit $M \rightarrow \infty$, which removes the Hamiltonian term from the master equation.

\subsubsection{Relevance of the quantum correction term}

We now estimate the relative weight of the two contributions at the right-hand side of Eq. (7.44), so as to gain an insight on the relevance of the quantum correction term with coefficient $D_{x x}$. In order to do that we recall the expressions (5.18) and (5.19) for the diffusion coefficients in terms of the characteristic thermal scales. One thus obtains an expression involving the friction constant $\eta$, as well as the temperature and the mass of the test particle,

$$
\begin{aligned}
\left\langle\mathbf{P}\left|\rho_{t}\right| \mathbf{P}^{\prime}\right\rangle= & \exp \left(-\frac{\beta}{16} \frac{\left(\mathbf{P}-\mathbf{P}^{\prime}\right)^{2}}{M}\left[1+\frac{4}{3}\left(\frac{t}{\beta \hbar}\right)^{2}\right] \eta t\right) \exp \left(-\frac{i}{\hbar} \frac{P^{2}-P^{\prime 2}}{2 M} t\right) \\
& \times\left(\frac{\beta}{4 \pi M \eta t}\right)^{3 / 2} \int \mathrm{d} \mathbf{Q} \exp \left(-\frac{\beta Q^{2}}{4 M \eta t}\right)\left\langle\mathbf{P}-\mathbf{Q}\left|\rho_{0}\right| \mathbf{P}^{\prime}-\mathbf{Q}\right\rangle
\end{aligned}
$$


This equation tells us that momentum coherences are exponentially suppressed for $\mathbf{P}-\mathbf{P}^{\prime}$ exceeding the thermal momentum spread $\left\langle P^{2}\right\rangle_{\beta}=3 M / \beta$. The first factor corresponding to the quantum term, however, is the dominating contribution only for times $t \lesssim \beta \hbar$, while for longer times the effect due to the spatial dispersion dominates and grows very quickly with elapsing time.

\section{The dynamic structure factor}

We now assume a slightly different point of view on the classical and the quantum linear Boltzmann equation, by rewriting it in a way which puts into evidence the statistical properties of the gas, as well as the role of the energy and momentum transfer. This is achieved by expressing the collision kernel in terms of the dynamic structure factor of the background gas, a two-point correlation function of its density fluctuations. This quantity, which is popular in the analysis of experiments where microscopic probes scatter off macroscopic samples, is defined as the Fourier transform of the time dependent density autocorrelation function of the gas with respect to both the energy transfer and the momentum transfer of a single collision. Its appearance in the quantum linear Boltzmann equation suggests a natural interpretation of the ensuing dynamics as driven by the density fluctuations in the medium, in accordance with Einstein's understanding of Brownian motion [181. We emphasize that the results discussed in this section are valid for a MaxwellBoltzmann distribution for the gas particles, while they generally rely on further assumptions for more general distributions.

\subsection{An alternative formulation of the linear Boltzmann equation}

\subsubsection{The classical expression}

Let us go back to the classical linear Boltzmann equation written in terms of an explicit energy conservation as in Eq. (3.1). In Sect. 3it serves as a starting point for the heuristic motivation of the quantum Boltzmann equation, where a decisive step is the decomposition of the momenta of gas and test particle into components parallel and perpendicular to the momentum transfer $\mathbf{Q}$. This permits one to express the energy conserving delta function in the classical linear Boltzmann equation in terms of the parallel momentum components only. In the scattering cross-section, in contrast, only momentum components perpendicular to the momentum transfer $\mathbf{Q}$ appear, as can be seen in Eq. (A.8).

We can now observe that the Maxwell-Boltzmann distribution (2.6) is invariant under rotations and that it factorizes in Cartesian coordinates. This implies that for any fixed direction $\mathbf{Q}$ one can write

$$
\mu_{\beta}(\mathbf{p})=\mu_{\beta}^{(2 d)}\left(\mathbf{p}_{\perp \mathbf{Q}}\right) \mu_{\beta}^{(1 d)}\left(\mathbf{p}_{\| \mathbf{Q}}\right)
$$

where

$$
\begin{aligned}
& \mu_{\beta}^{(2 d)}(\mathbf{p})=\frac{\beta}{2 \pi m} \exp \left(-\frac{\beta \mathbf{p}^{2}}{2 m}\right) \\
& \mu_{\beta}^{(1 d)}(\mathbf{p})=\sqrt{\frac{\beta}{2 \pi m}} \exp \left(-\frac{\beta \mathbf{p}^{2}}{2 m}\right)
\end{aligned}
$$

denote probability densities in two and one dimensions, respectively. It follows that also the momentum distribution can be split into factors depending either on the parallel or the perpendicular components of the gas momenta with respect to the momentum transfer $\mathbf{Q}$.

Exploiting these facts, one can perform the integral over the parallel component of the gas momentum in the linear Boltzmann equation Eq. (A.8). This can be done without specifying the scattering cross-section since the latter depends only on the orthogonal components of the gas momenta. One thus obtains the compact expression 


$$
\begin{aligned}
\partial_{t}^{\text {coll }} f(\mathbf{P})= & \frac{n_{\text {gas }}}{m_{*}^{2}} \int \mathrm{d} \mathbf{Q} \int_{\mathbf{Q}^{\perp}} \mathrm{d} \mathbf{k}_{\perp} \mu_{\beta}^{(2 d)}\left(\mathbf{k}_{\perp}\right) \sigma\left(\operatorname{rel}\left(\mathbf{k}_{\perp}, \mathbf{P}_{\perp \mathbf{Q}}\right)-\frac{\mathbf{Q}}{2}, \operatorname{rel}\left(\mathbf{k}_{\perp}, \mathbf{P}_{\perp \mathbf{Q}}\right)+\frac{\mathbf{Q}}{2}\right) \\
& \times\left[S_{\mathrm{MB}}(\mathbf{Q}, \mathbf{P}-\mathbf{Q}) f(\mathbf{P}-\mathbf{Q})-S_{\mathrm{MB}}(\mathbf{Q}, \mathbf{P}) f(\mathbf{P})\right],
\end{aligned}
$$

upon defining

$$
\begin{aligned}
S_{\mathrm{MB}}(\mathbf{Q}, \mathbf{P}) & \equiv \int \mathrm{d} \mathbf{k} \mu_{\beta}(\mathbf{k}) \delta\left(\frac{(\mathbf{P}+\mathbf{Q})^{2}}{2 M}+\frac{(\mathbf{k}-\mathbf{Q})^{2}}{2 m}-\frac{P^{2}}{2 M}-\frac{k^{2}}{2 m}\right) \\
& =\int_{\mathbf{Q} \|} \mathrm{d} \mathbf{k}_{\|} \mu_{\beta}^{(1 d)}\left(\mathbf{k}_{\|}\right) \delta\left(\frac{Q^{2}}{2 m_{*}}-\frac{1}{m_{*}} \mathbf{Q} \cdot \operatorname{rel}\left(\mathbf{k}_{\|}, \mathbf{P}_{\| \mathbf{Q}}\right)\right) .
\end{aligned}
$$

The second line follows from the identity Eq. (A.4) and the normalization is fixed in Eq. (8.3). As will be discussed in Sect.8.2, the function $S_{\mathrm{MB}}(\mathbf{Q}, \mathbf{P})$ is the dynamic structure factor of a gas of Maxwell-Boltzmann particles.

By further introducing an averaged scattering cross-section, involving the two-dimensional distribution (8.2) over the perpendicular gas momenta,

$$
\sigma_{\mathrm{av}}\left(\mathbf{P}_{\perp \mathbf{Q}}, \mathbf{Q}\right) \equiv \int_{\mathbf{Q}^{\perp}} \mathrm{d} \mathbf{k}_{\perp} \mu_{\beta}^{(2 d)}\left(\mathbf{k}_{\perp}\right) \sigma\left(\operatorname{rel}\left(\mathbf{k}_{\perp}, \mathbf{P}_{\perp \mathbf{Q}}\right)-\frac{\mathbf{Q}}{2}, \operatorname{rel}\left(\mathbf{k}_{\perp}, \mathbf{P}_{\perp \mathbf{Q}}\right)+\frac{\mathbf{Q}}{2}\right)
$$

Equation (8.4) assumes the compact form

$$
\partial_{t}^{\text {coll }} f(\mathbf{P})=\frac{n_{\text {gas }}}{m_{*}^{2}} \int \mathrm{d} \mathbf{Q} \sigma_{\text {av }}\left(\mathbf{P}_{\perp \mathbf{Q}}, \mathbf{Q}\right)\left[S_{\mathrm{MB}}(\mathbf{Q}, \mathbf{P}-\mathbf{Q}) f(\mathbf{P}-\mathbf{Q})-S_{\mathrm{MB}}(\mathbf{Q}, \mathbf{P}) f(\mathbf{P})\right] .
$$

By exploiting the factorized expression of the gas distribution function Eq. 8.1) we thus obtained an expression of the classical linear Boltzmann equation which involves only an integral over the momentum transfer $\mathbf{Q}$. Note that if the scattering cross-section only depends on the momentum transfer, as is the case in the Born approximation, the $\mathbf{k}_{\perp}$-integration can be done even in the absence of a factorization property like Eq. (8.1). The averaged scattering cross-section (8.6) then coincides with the proper cross-section.

The integral in Eq. 8.5) can be calculated explicitly, leading to an expression which is best stated in terms of the momentum $\mathbf{Q}$ and the energy $E$ transferred to the test particle, whose momentum prior to the collision is $\mathbf{P}$. We take these quantities as positive if the test particle gains momentum or energy. According to Eq. (4.11) the energy transfer is $E(\mathbf{Q}, \mathbf{P})=Q^{2} /(2 M)+\mathbf{Q} \cdot \mathbf{P} / M$, such that it depends only on $\mathbf{P}_{\| \mathbf{Q}}$. The function $S_{\mathrm{MB}}$ can therefore be expressed equivalently as a function of $\mathbf{Q}$ and $\mathbf{P}_{\| \mathbf{Q}}$, or of $\mathbf{Q}$ and $E(\mathbf{Q}, \mathbf{P})$,

$$
S_{\mathrm{MB}}(\mathbf{Q}, \mathbf{P})=S_{\mathrm{MB}}(\mathbf{Q}, E(\mathbf{Q}, \mathbf{P}))=\sqrt{\frac{\beta m}{2 \pi}} \frac{1}{Q} \exp \left(-\frac{\beta}{8 m} \frac{\left(Q^{2}+2 m E(\mathbf{Q}, \mathbf{P})\right)^{2}}{Q^{2}}\right) .
$$

This exact expression implies in particular

$$
\frac{m}{Q} \mu_{\beta}\left(\mathbf{p}_{\perp \mathbf{Q}}+\frac{m}{m_{*}} \frac{\mathbf{Q}}{2}+\frac{m}{M} \mathbf{P}_{\| \mathbf{Q}}\right)=\mu_{\beta}^{(2 d)}\left(\mathbf{p}_{\perp \mathbf{Q}}\right) S_{\mathrm{MB}}\left(\mathbf{Q}, \mathbf{P}_{\| \mathbf{Q}}\right),
$$

where we stress explicitly that the function $S_{\mathrm{MB}}$ depends on $\mathbf{P}$ only through $\mathbf{P}_{\| \mathbf{Q}}$. Using Eq. (8.9) one immediately finds that Eq. (8.4) and Eq. (3.2) are two equivalent forms of the same equation.

\subsubsection{Generalization to the quantum case}

The particular form (8.4) of the classical linear Boltzmann equation suggests an analogous way of writing the quantum linear Boltzmann equation [58|42]47|182]183, 


$$
\begin{aligned}
\mathcal{L} \rho= & \frac{n_{\text {gas }}}{m_{*}^{2}} \int \mathrm{d} \mathbf{Q} \int_{\mathbf{Q}^{\perp}} \mathrm{d} \mathbf{k}_{\perp} \mu_{\beta}^{(2 d)}\left(\mathbf{k}_{\perp}\right) \\
& \times\left[f\left(\operatorname{rel}\left(\mathbf{k}_{\perp}, \mathrm{P}_{\perp \mathbf{Q}}\right)-\frac{\mathbf{Q}}{2}, \operatorname{rel}\left(\mathbf{k}_{\perp}, \mathrm{P}_{\perp \mathbf{Q}}\right)+\frac{\mathbf{Q}}{2}\right) \mathrm{e}^{i \mathbf{Q} \cdot \mathbf{X} / \hbar} \sqrt{S_{\mathrm{MB}}(\mathbf{Q}, \mathrm{P})} \rho\right. \\
& \times \sqrt{S_{\mathrm{MB}}(\mathbf{Q}, \mathrm{P})} \mathrm{e}^{-i \mathbf{Q} \cdot \times / \hbar} f^{\dagger}\left(\operatorname{rel}\left(\mathbf{k}_{\perp}, \mathrm{P}_{\perp \mathbf{Q}}\right)-\frac{\mathbf{Q}}{2}, \operatorname{rel}\left(\mathbf{k}_{\perp}, \mathrm{P}_{\perp \mathbf{Q}}\right)+\frac{\mathbf{Q}}{2}\right) \\
& \left.-\frac{1}{2}\left\{S_{\mathrm{MB}}(\mathbf{Q}, \mathrm{P})\left|f\left(\operatorname{rel}\left(\mathbf{k}_{\perp}, \mathrm{P}_{\perp \mathbf{Q}}\right)-\frac{\mathbf{Q}}{2}, \operatorname{rel}\left(\mathbf{k}_{\perp}, \mathrm{P}_{\perp \mathbf{Q}}\right)+\frac{\mathbf{Q}}{2}\right)\right|^{2}, \rho\right\}\right] .
\end{aligned}
$$

This is exactly equivalent to Eq. (2.2) for a gas of Maxwell-Boltzmann particles, described by Eq. (2.6), as can easily be seen using Eq. (8.9). Note that the square root in Eq. (8.10) is well defined since $S_{\mathrm{MB}}$ is positive, which is a general property of the dynamic structure factor, as discussed in the following section.

\subsection{Properties of the dynamic structure factor}

\subsubsection{Expression in terms of the density correlation function}

Let us consider a quantum many-body system, e.g. the gas of free particles considered in the previous paragraph, described in second quantization by a field operator $\hat{\psi}(\mathbf{x})$, satisfying canonical commutation or anti-commutation rules. One can then consider the Fourier transformation of the operator density $\hat{n}(\mathbf{x})=$ $\hat{\psi}^{\dagger}(\mathbf{x}) \hat{\psi}(\mathbf{x})$ given by

$$
\hat{\rho}_{\mathbf{Q}}=\int \mathrm{d} \mathbf{x} \mathrm{e}^{-i \mathbf{Q} \cdot \mathbf{x} / \hbar} \hat{n}(\mathbf{x}) .
$$

The associated spectral function

$$
S(\mathbf{Q}, E)=\frac{1}{2 \pi \hbar} \frac{1}{N} \int \mathrm{d} t \mathrm{e}^{i E t / \hbar}\left\langle\hat{\rho}_{\mathbf{Q}}^{\dagger} \hat{\rho}_{\mathbf{Q}}(t)\right\rangle,
$$

with $N$ the total number of particles, is known as dynamic structure factor [184[185]186], though other names such as "spectral density function", "scattering function" and "scattering law" are also used in the literature. It can be expressed in general as the Fourier transform with respect to energy and momentum transfer of the time dependent density autocorrelation function

$$
G(\mathbf{x}, t)=\frac{1}{N} \int \mathrm{d} \mathbf{y}\langle\hat{n}(\mathbf{y}) \hat{n}(\mathbf{x}+\mathbf{y}, t)\rangle
$$

as

$$
S(\mathbf{Q}, E)=\frac{1}{2 \pi \hbar} \int \mathrm{d} t \int \mathrm{d} \mathbf{x} e^{i(E t-\mathbf{Q} \cdot \mathbf{x}) / \hbar} G(\mathbf{x}, t) .
$$

By evaluating the dynamic structure factor for the case of a system of free distinguishable particles one obtains, recalling Eq. (4.11) for the energy transfer,

$$
S(\mathbf{Q}, E(\mathbf{Q}, \mathbf{P}))=\frac{1}{n_{\text {gas }}} \int \frac{\mathrm{d} \mathbf{p}}{(2 \pi \hbar)^{3}}\left\langle\hat{n}_{\mathbf{p}}\right\rangle \delta\left(E(\mathbf{Q}, \mathbf{P})+\frac{(\mathbf{p}-\mathbf{Q})^{2}}{2 m}-\frac{p^{2}}{2 m}\right) .
$$

Here $\left\langle\hat{n}_{\mathbf{p}}\right\rangle$ denotes the expectation value of $\hat{n}_{\mathbf{p}}$, i.e. the mean number of particles with momentum $\mathbf{p}$. If we consider a Maxwell-Boltzmann distribution, such that

$$
\left\langle\hat{n}_{\mathbf{p}}\right\rangle \rightarrow n_{\text {gas }}(2 \pi \hbar)^{3} \mu_{\beta}(\mathbf{p}),
$$

one comes back to the expression introduced in Eq. (8.8) (see e.g. [47[187]). 
It thus appears that the dynamic structure factor $S_{\mathrm{MB}}$, which can be used to express the scattering kernel of the linear Boltzmann equation (8.10), has an important and transparent physical meaning. It is the Fourier transform of the correlation function of the density fluctuations in the medium, reflecting the molecular nature of the gas, which determines the collisional interaction between test particle and gas particles. It thus embodies in a suitable correlation function the original intuition by Einstein which led to the correct understanding of Brownian motion: the dynamics of the test particle is driven by collisions taking place due to the discrete nature of the medium, whose density fluctuations provide the coupling mechanism.

\subsubsection{Connection to the laboratory-frame scattering cross-section}

The dynamic structure factor is particularly useful in describing the scattering of microscopic probes off macroscopic samples, since it allows one to express the energy dependent cross-section in terms of a correlation function of the many-body system [188 185]. It was in fact first introduced by van Hove in order to describe neutron scattering [189].

Using Fermi's golden rule and the Born approximation one can show that the laboratory-frame crosssection $\Sigma$ for the scattering of a test particle off a many-body system is characterized by the dynamic structure factor $S(\mathbf{Q}, E)$ of the many-body system [131]

$$
\frac{\mathrm{d}^{2} \Sigma}{\mathrm{d} \Omega_{P^{\prime}} \mathrm{d} E_{P^{\prime}}}=\frac{M^{2}}{4 \pi^{2} \hbar^{4}} \frac{P^{\prime}}{P}|\tilde{V}(\mathbf{Q})|^{2} S(\mathbf{Q}, E)=\frac{M^{2}}{m_{*}^{2}} \frac{P^{\prime}}{P}\left|f_{B}(\mathbf{Q})\right|^{2} S(\mathbf{Q}, E) .
$$

Here a scattering event is considered in which a particle of mass $M$ changes its momentum from $\mathbf{P}$ to $\mathbf{P}^{\prime}=\mathbf{P}+\mathbf{Q}$, absorbing an energy $E$ according to (4.11), and $\tilde{V}(\mathbf{Q})$ is the Fourier transform of the two-body interaction potential

$$
\tilde{V}(\mathbf{Q})=\int \mathrm{d} \mathbf{x} \mathrm{e}^{i \mathbf{Q} \cdot \mathbf{x} / \hbar} V(\mathbf{x})
$$

related through Eq. (2.15) to the scattering amplitude in Born approximation.

More generally, provided multiple scattering effects can be neglected, one has [188]

$$
\frac{\mathrm{d}^{2} \Sigma}{\mathrm{d} \Omega_{P^{\prime}} \mathrm{d} E_{P^{\prime}}}=\frac{\mathrm{d} \Sigma}{\mathrm{d} \Omega_{P^{\prime}}} S(\mathbf{Q}, E)
$$

where $\mathrm{d} \Sigma / \mathrm{d} \Omega_{P^{\prime}}$ describes the single scattering event in the laboratory frame.

\subsubsection{Detailed balance and the stationary solution}

For a strongly interacting many-body quantum system the dynamic structure factor is no longer analytically tractable as in Eq. (8.8), but it can only be measured in suitable scattering experiments. However, due to its very definition the dynamic structure factor acquires many interesting general properties. First of all, it is always positive as a consequence of Bochner's theorem [152, because the density autocorrelation function Eq. (8.13) is a positive definite function. This property is to be expected on physical grounds, since the dynamic structure factor is directly proportional to a scattering cross-section according to Eq. (8.17). The dynamic structure factor further obeys the so-called detailed balance condition

$$
S(\mathbf{Q}, E)=\mathrm{e}^{-\beta E} S(-\mathbf{Q},-E)
$$

which is the crucial property for ensuring the existence of a stationary solution of both the classical and the quantum linear Boltzmann equation. In order to confirm this property one has to consider Eq. (8.12) and to observe that $\hat{\rho}_{\mathbf{Q}}^{\dagger}=\hat{\rho}_{-\mathbf{Q}}$, as follows directly from the definition Eq. (8.11), and to exploit the fact that the expectation value is obtained with respect to the equilibrium state of the many-body system $\rho_{\mathrm{EQ}} \propto \mathrm{e}^{-\beta H}$, where $H$ is the full Hamiltonian. The property Eq. 8.20 therefore holds generally, for any many-body system in a thermal equilibrium state.

In our case, where the energy transfer can be expressed by Eq. (4.11), the detailed balance condition can also be written as 


$$
S(\mathbf{Q}, \mathbf{P})=\mathrm{e}^{-\beta E(\mathbf{Q}, \mathbf{P})} S(-\mathbf{Q}, \mathbf{P}+\mathbf{Q}) .
$$

It is now simple to prove that Eq. (8.21) ensures the existence of a stationary solution of the canonical form $\rho_{\mathrm{EQ}}(\mathbf{P}) \propto \exp \left(-\beta P^{2} /(2 M)\right)$ for both the classical and the quantum linear Boltzmann equation. For $f(\mathbf{P}) \rightarrow \rho_{\mathrm{EQ}}(\mathbf{P})$ the expression

$$
S_{\mathrm{MB}}(\mathbf{Q}, \mathbf{P}-\mathbf{Q}) \rho_{\mathrm{EQ}}(\mathbf{P}-\mathbf{Q})-S_{\mathrm{MB}}(\mathbf{Q}, \mathbf{P}) \rho_{\mathrm{EQ}}(\mathbf{P})
$$

is odd in $\mathbf{Q}$, such that Eq. (8.4) implies $\partial_{t}^{\text {coll }} \rho_{\mathrm{EQ}}(\mathbf{P})=0$. Similarly, in the quantum case the expression Eq. (8.10) for an operator $\rho=\nu(\mathrm{P})$, which is only a function of the momentum operator $\mathrm{P}$, simply reads

$$
\begin{aligned}
\mathcal{L} \nu(\mathrm{P})= & \frac{n_{\text {gas }}}{m_{*}^{2}} \int \mathrm{d} \mathbf{Q} \int_{\mathbf{Q}^{\perp}} \mathrm{d} \mathbf{k}_{\perp} \mu_{\beta}^{(2 d)}\left(\mathbf{k}_{\perp}\right)\left|f\left(\operatorname{rel}\left(\mathbf{k}_{\perp}, \mathrm{P}_{\perp \mathbf{Q}}\right)-\frac{\mathbf{Q}}{2}, \operatorname{rel}\left(\mathbf{k}_{\perp}, \mathrm{P}_{\perp \mathbf{Q}}\right)+\frac{\mathbf{Q}}{2}\right)\right|^{2} \\
& \times\left[S_{\mathrm{MB}}(\mathbf{Q}, \mathrm{P}-\mathbf{Q}) \nu(\mathrm{P}-\mathbf{Q})-S_{\mathrm{MB}}(\mathbf{Q}, \mathrm{P}) \nu(\mathrm{P})\right] .
\end{aligned}
$$

For the case $\nu(\mathrm{P}) \rightarrow \nu_{\mathrm{EQ}}(\mathrm{P})$ the condition Eq. (8.21) again implies that the integrand is an odd function of Q, such that $\mathcal{L} \nu_{\mathrm{EQ}}(\mathrm{P})=0$. Note that the explicit expression Eq. (8.8) of $S_{\mathrm{MB}}$ is not relevant for the proof, which relies only on the property Eq. 8.21), valid for a generic medium.

As expected, also in the quantum case the existence and the form of the stationary solution do not depend on the scattering amplitude, even though it appears operator-valued. This strong correspondence with the classical case is due to the fact that the covariance under translations implies that the generator $\mathcal{L}$ leaves the algebra generated by the momentum operator invariant, as discussed in Sect. 4.3 .

\subsubsection{Fluctuation-dissipation relationship}

Let us now discuss the origin of the detailed balance property, beyond its direct calculation based on the definition of dynamic structure factor in Eq. (8.12). To this end, we have to touch upon the fluctuationdissipation theorem.

The dynamic structure factor can be directly related to the dynamic susceptibility studied in linear response theory. Denoting the imaginary part of the dynamic response function as $\chi^{\prime \prime}(\mathbf{Q}, E)$, one finds $[186$ ]

$$
S(\mathbf{Q}, E)=\frac{1}{\pi} \frac{1}{1-e^{\beta E}} \chi^{\prime \prime}(\mathbf{Q}, E) .
$$

Since the imaginary part of the dynamic susceptibility, which describes the dissipative part of the response, is by definition an odd function of energy, this relationship ensures that the dynamic structure factor obeys the detailed balance condition Eq. (8.20). We recall that, contrary to the usual perspective in linear response theory, we are here concerned with the reduced dynamics of the test particle, thus taking the momentum and energy transferred to the particle as positive.

Introducing the real correlation function of the gas medium

$$
\phi^{+}(\mathbf{Q}, t)=\frac{1}{\hbar N}\left\langle\left\{\hat{\rho}_{\mathbf{Q}}(t), \hat{\rho}_{\mathbf{Q}}^{\dagger}\right\}\right\rangle,
$$

for the fluctuations of the operator $\hat{\rho}_{\mathbf{Q}}$ given by Eq. 8.11), the fluctuation-dissipation relationship can be formulated in terms of the dynamic structure factor as follows [184 56 ]

$$
\phi^{+}(\mathbf{Q}, t)=-\frac{1}{\hbar} \int_{-\infty}^{+\infty} \mathrm{d} E \exp \left(i \frac{E}{\hbar} t\right) \operatorname{coth}\left(\frac{\beta}{2} E\right)\left(1-e^{\beta E}\right) S(\mathbf{Q}, E)
$$

This is a consequence of the definition of the dynamic structure factor Eq. (8.12) as well as Eq. (8.25). When expressed in terms of the imaginary part of the dynamic susceptibility according to Eq. (8.24) this identity gives back the usual formulation of the fluctuation-dissipation theorem, once again taking as positive the energy when transferred to the test particle. 
In this spirit, the expression (8.17) for the laboratory-frame cross-section can be viewed as a formulation of the fluctuation-dissipation relationship. The test particle experiences dissipation due to energy and momentum transfer processes described by the cross-section on the left-hand side of Eq. (8.17), which is related on the right-hand side to the equilibrium density fluctuations, characterized by the dynamic structure factor.

\subsection{Extension to different reservoirs}

In the previous sections, we considered the explicit expression of the dynamic structure factor for an ideal gas described by the Maxwell-Boltzmann distribution. The latter implies that the gas particles are either distinguishable or that the gas temperature is sufficiently high such that effects due to their indistinguishability can be neglected. We note that the result (8.8) is the same in the classical and the quantum calculation [184.

For the case of an ideal gas, one can calculate the exact dynamic structure factor also by taking into account the effect of quantum statistics. Starting from the density autocorrelation function Eq. (8.13) for a gas of identical bosons or fermions it takes the form

$$
S_{\mathrm{BF}}(\mathbf{Q}, E(\mathbf{Q}, \mathbf{P}))=\frac{1}{n_{\text {gas }}} \int \frac{\mathrm{d} \mathbf{p}}{(2 \pi \hbar)^{3}}\left\langle\hat{n}_{\mathbf{p}}\right\rangle_{\mathrm{BF}}\left(1 \pm\left\langle\hat{n}_{\mathbf{p}}\right\rangle_{\mathrm{BF}}\right) \delta\left(E(\mathbf{Q}, \mathbf{P})+\frac{(\mathbf{p}-\mathbf{Q})^{2}}{2 m}-\frac{p^{2}}{2 m}\right),
$$

which should be compared to Eq. (8.15). Here the upper sign stands for Bose-Einstein and the lower one for Fermi-Dirac statistics, respectively. The corresponding distribution functions

$$
\left\langle\hat{n}_{\mathbf{p}}\right\rangle_{\mathrm{BF}}=\frac{1}{z^{-1} \mathrm{e}^{\beta \frac{\mathbf{p}^{2}}{2 M}} \mp 1}
$$

are characterized by the fugacity $z=\exp (\beta \mu)$. The integral (8.27) can be done, leading to [47]

$$
S_{\mathrm{BF}}(\mathbf{Q}, E(\mathbf{Q}, \mathbf{P}))=\frac{1}{n_{\mathrm{gas}}} \frac{2 \pi m^{2}}{\beta(2 \pi \hbar)^{3}} \frac{1}{Q} \frac{ \pm 1}{1-\mathrm{e}^{\beta E(\mathbf{Q}, \mathbf{P})}} \log \left[\frac{1 \mp z \exp \left(-\frac{\beta}{8 m} \frac{\left(Q^{2}+2 m E(\mathbf{Q}, \mathbf{P})\right)^{2}}{Q^{2}}\right)}{1 \mp z \exp \left(-\frac{\beta}{8 m} \frac{\left(Q^{2}-2 m E(\mathbf{Q}, \mathbf{P})\right)^{2}}{Q^{2}}\right)}\right] .
$$

In the limit of small fugacity $z \rightarrow n_{\text {gas }}\left(2 \pi \hbar^{2} \beta / m\right)^{3 / 2}$, corresponding to negligible degeneracy, the latter expression goes back to Eq. (8.15). As one can also check directly, $S_{\mathrm{BF}}(\mathbf{Q}, E)$ obeys the detailed balance condition 8.20 for both statistics.

Derivations of the quantum linear Boltzmann equation in the weak-coupling limit, in which a thermal reservoir of identical quantum particles is considered, have been obtained in [58] for the case of a bosonic reservoir, and more generally in 42 , leading to

$$
\mathcal{L} \rho=\frac{n_{\text {gas }}}{m_{*}^{2}} \int \mathrm{d} \mathbf{Q}\left|f_{B}(\mathbf{Q})\right|^{2}\left[\mathrm{e}^{i \mathbf{Q} \cdot \mathbf{X} / \hbar} \sqrt{S_{\mathrm{BF}}(\mathbf{Q}, \mathrm{P})} \rho \sqrt{S_{\mathrm{BF}}(\mathbf{Q}, \mathbf{P})} \mathrm{e}^{-i \mathbf{Q} \cdot \mathbf{X} / \hbar}-\frac{1}{2}\left\{S_{\mathrm{BF}}(\mathbf{Q}, \mathrm{P}), \rho\right\}\right]
$$

This result is the quantum counterpart of a classical linear Boltzmann equation, which is modified in order to account for statistical corrections. The latter can be obtained upon setting $\mu_{\mathrm{BF}}(\mathbf{p})=\left\langle\hat{n}_{\mathbf{p}}\right\rangle_{\mathrm{BF}} /\left(n_{\mathrm{gas}}(2 \pi \hbar)^{3}\right)$ and replacing $\mu_{\beta}(\mathbf{p})$ in Eq. (3.1) by $\mu_{\mathrm{BF}}(\mathbf{p})\left(1 \pm n_{\text {gas }}(2 \pi \hbar)^{3} \mu_{\mathrm{BF}}\left(\mathbf{p}^{\prime}\right)\right)$, which leads to

$$
\begin{aligned}
\partial_{t}^{\text {coll }} f(\mathbf{P})= & \frac{n_{\text {gas }}}{m_{*}^{2}} \int \mathrm{d} \mathbf{P}^{\prime} \mathrm{d} \mathbf{p}^{\prime} \mathrm{d} \mathbf{p} \delta\left(\frac{P^{\prime 2}}{2 M}+\frac{p^{\prime 2}}{2 m}-\frac{P^{2}}{2 M}-\frac{p^{2}}{2 m}\right) \delta^{3}\left(\mathbf{P}^{\prime}+\mathbf{p}^{\prime}-\mathbf{P}-\mathbf{p}\right) \\
& \times \sigma\left(\operatorname{rel}(\mathbf{p}, \mathbf{P}), \operatorname{rel}\left(\mathbf{p}^{\prime}, \mathbf{P}^{\prime}\right)\right)\left[\mu_{\mathrm{BF}}\left(\mathbf{p}^{\prime}\right)\left(1 \pm n_{\text {gas }}(2 \pi \hbar)^{3} \mu_{\mathrm{BF}}(\mathbf{p})\right) f\left(\mathbf{P}^{\prime}\right)\right. \\
& \left.-\mu_{\mathrm{BF}}(\mathbf{p})\left(1 \pm n_{\text {gas }}(2 \pi \hbar)^{3} \mu_{\mathrm{BF}}\left(\mathbf{p}^{\prime}\right)\right) f(\mathbf{P})\right]
\end{aligned}
$$

The statistical corrections in this modified classical linear Boltzmann equation are the analog of the modifications introduced by Uehling and Uhlenbeck to the original classical equation [9]. They express the fact 
that quantum correlations in the gas can be relevant even in the absence of any self-interactions, just because the occupancy of the different momentum states may enhance or suppress the related scattering rates due to statistics.

We mention that a simplified form of the master equation (8.10) was used in [58] to study how the effects of quantum statistics in a Boltzmann gas affect the reduction of visibility in the interference fringes due to collisional decoherence.

Finally, it is interesting to note that the comparison between Eq. (8.30) and Eq. (8.10) suggests a natural heuristic approach to extend the quantum linear Boltzmann equation to real, interacting gases. In place of the dynamic structure factor of the ideal gas one just uses the relevant expression of the interacting gas. Even if an exact analytic evaluation of the dynamic structure factor is in general impossible for a truly interacting many-body system, one may rely either on experimental data from scattering experiments or on a phenomenological ansatz, constrained by the requirement of the detailed balance condition. This strategy is straightforward for the weak-coupling form (8.30) of the master equation. However, complications arise if the interaction with the gas is to be treated beyond the Born approximation, as in Eq. (8.10), once the momentum distribution in the gas no longer factorizes as in Eq. (8.1).

\subsection{Inclusion of internal degrees of freedom}

The quantum linear Boltzmann equation provides a realistic description for the dynamics of a massive test particle traveling through a dilute gas of free particles, and its predictions in the quantum framework have been tested in recent experiments relevant for atom interferometry [37/39,155/38]. It is however natural to consider extensions of the equation, allowing one to cope with a wider experimental scenario.

In the first instance both test particle and gas particles have been considered as pointlike particles, whose only relevant degrees of freedom are those connected to the center of mass. This is obviously not necessarily the case and one should consider a proper quantum treatment of both internal and center of mass degrees of freedom.

A hybrid equation, describing the internal degrees of freedom within quantum mechanics, and the center of mass degrees of freedom as classical variables, was recently introduced under the name "Bloch-Boltzmann equation" 190191. It combines the master equation for the gas-induced incoherent dynamics of a $N$-level system of internal states with the classical linear Boltzmann equation. This Bloch-Boltzmann equation thus accounts for the possibility of inelastic collisions connecting the various possible scattering channels, which can be of relevance e.g. for spectroscopic experiments [192, transport phenomena, or more refined interferometric experiments testing decoherence.

A microscopic derivation of the effect of the collisional dynamics on the internal degrees of freedom of a very massive test particle at rest was considered in 6965. A fully quantum description of both internal and center of mass degrees of freedom of a quantum test particle interacting through collisions with a gas has been considered in 193, leading to an equation called "quantum Bloch-Boltzmann equation". From this equation all the others can be obtained as limiting situations in which one of the two kinds of degrees of freedom is traced over or treated classically. In this framework, a rich phenomenology of dynamical situations can be considered, including non-Markovian effects described by means of the so-called generalized Lindblad structure 194195196197.

The "quantum Bloch-Boltzmann equation" can be written in Lindblad form in close analogy to the linear Boltzmann equation (2.1). However, the statistical operator $\rho$ now acts on the Hilbert space describing both internal and center of mass degrees of freedom. The correction to the free Hamiltonian, which is the analog of Eq. (2.5), now reads

$$
\begin{gathered}
\mathrm{H}_{\mathrm{n}}=-2 \pi \hbar^{2} \frac{n_{\text {gas }}}{m_{*}} \sum_{i j} \int \mathrm{d} \mathbf{p} \mu(\mathbf{p}) \operatorname{Re}\left[f_{i j}(\operatorname{rel}(\mathbf{p}, \mathrm{P}), \operatorname{rel}(\mathbf{p}, \mathrm{P}))\right] \otimes \mathrm{E}_{i j}, \\
\mathcal{E}_{i j}=0
\end{gathered}
$$


where $\mathrm{E}_{i j}=|i\rangle\langle j|$ provides a basis of operators in the space of the internal degrees of freedom, and $\mathcal{E}_{i j}=\hbar \omega_{i}-$ $\hbar \omega_{j}$ are the possible transition energies. The multi-channel scattering amplitudes, denoted by $f_{i j}\left(\mathbf{p}_{f}, \mathbf{p}_{i}\right) \equiv$ $f\left(\mathbf{p}_{i}, j \rightarrow \mathbf{p}_{f}, i\right)$, describe a transition from an in-state with labels $\mathbf{p}_{i}, j$ to an out-state with labels $\mathbf{p}_{f}, i$.

The incoherent part of the master equation can be written analogous to Eq. (2.2). It takes the form

$$
\begin{aligned}
\mathcal{L} \rho= & \sum_{\mathcal{E}} \int \mathrm{d} \mathbf{Q} \int_{\mathbf{Q}^{\perp}} \mathrm{d} \mathbf{k}_{\perp}\left[\mathrm{e}^{i \mathbf{Q} \cdot \mathbf{X} / \hbar} L\left(\mathbf{k}_{\perp}, \mathrm{P} ; \mathbf{Q}, \mathcal{E}\right) \rho L^{\dagger}\left(\mathbf{k}_{\perp}, \mathrm{P} ; \mathbf{Q}, \mathcal{E}\right) \mathrm{e}^{-i \mathbf{Q} \cdot \mathbf{X} / \hbar}\right. \\
& \left.-\frac{1}{2}\left\{\rho, L^{\dagger}\left(\mathbf{k}_{\perp}, \mathrm{P} ; \mathbf{Q}, \mathcal{E}\right) L\left(\mathbf{k}_{\perp}, \mathrm{P} ; \mathbf{Q}, \mathcal{E}\right)\right\}\right]
\end{aligned}
$$

where, apart from the momentum integrations, there is an additional sum over a discrete index $\mathcal{E}$, labeling the energy transfer to the internal degrees of freedom in case of inelastic scattering.

The function $L$, which describes the effect of the single collisions, is given by

$$
\begin{aligned}
L(\mathbf{p}, \mathbf{P} ; \mathbf{Q}, \mathcal{E})= & \sum_{i j} f_{i j}\left(\operatorname{rel}\left(\mathbf{p}_{\perp \mathbf{Q}}, \mathbf{P}_{\perp \mathbf{Q}}\right)-\frac{\mathbf{Q}}{2}+\frac{\mathcal{E}_{i j}}{Q^{2} / m_{*}} \mathbf{Q}, \operatorname{rel}\left(\mathbf{p}_{\perp \mathbf{Q}}, \mathbf{P}_{\perp \mathbf{Q}}\right)+\frac{\mathbf{Q}}{2}+\frac{\mathcal{E}_{i j}}{Q^{2} / m_{*}} \mathbf{Q}\right) \\
& \mathcal{E}_{i j}=\mathcal{E} \\
& \times \sqrt{\frac{n_{\text {gas }} m}{m_{*}^{2} Q} \mu\left(\mathbf{p}_{\perp \mathbf{Q}}+\frac{m}{m_{*}} \frac{\mathbf{Q}}{2}+\frac{m}{M} \mathbf{P}_{\| \mathbf{Q}}+\frac{\mathcal{E}_{i j}}{Q^{2} / m} \mathbf{Q}\right)} \otimes \mathrm{E}_{i j},
\end{aligned}
$$

which should be compared to Eq. (2.3). This implies that the multi-channel scattering amplitudes $f_{i j}$ and the distribution function $\mu$ of the gas momenta appear again operator-valued in (8.33).

Equations (8.32)-(8.34) represent the natural generalization obtained by combining the two master equations for the quantum motion of a point particle and for the internal dynamics of a system at rest, respectively. It should be emphasized, however, that a physically stringent derivation of Eqs. 8.32)- (8.34) is still missing. It is a subject of current research whether this can be achieved by means of the monitoring approach described in [65, which was also instrumental in deriving the quantum linear Boltzmann equation.

\section{Conclusion}

In the present report we discussed a Lindblad master equation for the quantum motion of a test particle in an ambient gas, arguing that it is the natural quantum counterpart of the classical linear Boltzmann equation. This means that the gas-induced phenomena described by the equation are accounted for not just in a phenomenological sense, but are incorporated in a non-perturbative fashion by means of the exact scattering amplitudes. This microscopically realistic description of the individual scattering processes allows one to predict experimentally observable properties such as dissipation and decoherence rates directly from the microscopic interaction laws. At the same time, it explains why the structure of the equation is more complicated than most master equations used for the description of open quantum systems.

We presented a fairly straightforward, heuristic motivation for the particular form of the equation, referring the reader to the literature for a more stringent derivation [32 141]. It is remarkable that already two basic requirements serve to fix the master equation almost completely, namely the translation-covariance of its Lindblad structure and the compatibility with the classical linear Boltzmann equation for quantum states which are indistinguishable from classical ones. The remaining freedom in this heuristic approach can be settled by a plausibility argument, which is corroborated by the fact that the various limits that can be taken turn the equation into established master equations. In particular, a generalized Caldeira-Leggett master equation of quantum Brownian motion is obtained in the diffusive limit, whose Lindblad structure is as close as possible to the classical Kramers equation with microscopically defined friction and diffusion constants.

Some attention was devoted to analyzing the general structural features and symmetry properties of the equation, as well as the predicted approach to an equilibrium state. We saw that many of the relaxation 
and dissipation effects are shared with those of the classical linear Boltzmann equation, while pure quantum phenomena, such as collisional decoherence and the gas induced index of refraction for matter waves, are genuine results of the quantum version. A remarkable feature of the equation is that it accounts for the interplay of all relevant gas-induced phenomena, from the short-time decoherence dynamics to the approach towards equilibrium for asymptotically large times. At the same time, it is clearly impossible to state the general solution in an explicit form, even in the simplest case of s-wave scattering with a constant scattering length. However, we saw that the Lindblad structure of the master equation admits a stochastic unravelling of the master equation in terms of quantum trajectories, which is particularly transparent in the momentum basis.

The validity of the quantum linear Boltzmann equation is expected to cease in situations where the medium cannot be considered an ideal, homogeneous Maxwell-Boltzmann gas, or where the scattering interaction is inelastic. We discussed an alternative formulation of the master equation in terms of the dynamic structure factor of the gas. Apart from providing physical insights, it suggests a natural way of incorporating corrections due to quantum degeneracies and self-interactions in the gas, at least in the framework of the weak-coupling approximation. As for inelastic scattering, we described a natural multi-channel scattering extension of the equation, capable to account for the presence of internal degrees of freedom in the test particle.

A further important extension of the equation concerns the treatment of an inhomogeneous background gas, e.g. due to an external potential. In view of the corresponding classical equation, it seems natural to incorporate this by means of an operator-valued gas density $n_{\text {gas }}(\mathrm{X})$, rendering the transition rate $M_{\text {in }}$ a function of the position operator of the test particle. However, the non-commutativity of the position and momentum operators complicates this task considerably.

Finally, the limit of a dense and strongly self-interacting gas turns the background medium into a liquid. Clearly, the interactions with the test particle are then no longer described by statistically independent two-particle collisions, leading to a breakdown of the Markov assumption. In spite of many years of research, the field of non-Markovian open quantum dynamics is still in its infancy. However, it will certainly be of central relevance to cope with such non-Markovian effects in order to describe a truly quantum microscopic dynamics.

The long path leading to the present version of the quantum linear Boltzmann equation suggests that most of these improvements are still a long way ahead, and possibly will require a different mathematical framework. Nonetheless, they will be of great importance for a quantitative assessment of quantum transport and decoherence phenomena.

Acknowledgments

The work was partially supported by the Italian MIUR under PRIN2005 (BV) and by the Emmy Noether programme of Deutsche Forschungsgemeinschaft $(\mathrm{KH})$. We would like to thank A. Barchielli, H.-P. Breuer, L. Lanz, and J. E. Sipe for many useful discussions on the subject of this article.

\section{Appendix A. Classical formulation}

\section{A.1. The classical linear Boltzmann equation}

The classical linear Boltzmann equation can be written in quite different ways, which reflects its complicated structure and the many variables appearing in it [2]. The most compact and perhaps well-known form is given by

$$
\begin{aligned}
\partial_{t}^{\text {coll }} f(\mathbf{P})= & \int \mathrm{d} \mathbf{p} \int \mathrm{d} \Omega \sigma\left(\operatorname{rel}(\mathbf{p}, \mathbf{P}), \operatorname{rel}\left(\mathbf{p}^{\prime}, \mathbf{P}^{\prime}\right)\right) j_{\text {rel }}(\mathbf{p}, \mathbf{P}) \\
& \times\left[\mu\left(\mathbf{p}^{\prime}\right) f\left(\mathbf{P}^{\prime}\right)-\mu(\mathbf{p}) f(\mathbf{P})\right]
\end{aligned}
$$


where $j_{\text {rel }}(\mathbf{p}, \mathbf{P}) \equiv n_{\text {gas }}|\operatorname{rel}(\mathbf{p}, \mathbf{P})| / m_{*}$ denotes the current density in the relative motion. In this equation, the values of $\mathbf{p}^{\prime}$ and $\mathbf{P}^{\prime}$ are determined implicitly by the conservation of momentum, $\mathbf{p}^{\prime}+\mathbf{P}^{\prime}=\mathbf{p}+\mathbf{P}$, and energy, $\left|\operatorname{rel}\left(\mathbf{p}^{\prime}, \mathbf{P}^{\prime}\right)\right|=|\operatorname{rel}(\mathbf{p}, \mathbf{P})|$, as well as by the angles of rotation $\Omega$ which connect the relative momenta appearing in the differential cross-section $\sigma$. Moreover, we have only considered the collisional term.

However, it is much more convenient for our purposes to use a more explicit expression. In particular, is permits to clearly demonstrate the connection between the classical and the quantum version of the equation.

Let us start from the basic expression obtained by turning the original, non-linear Boltzmann equation into the linear version. By replacing one of the distribution functions $f(\mathbf{p})$ appearing in the bilinear collision term with the equilibrium distribution $\mu(\mathbf{p})$ of the gas particles, one gets an equation

$$
\begin{aligned}
\partial_{t}^{\text {coll }} f(\mathbf{P})= & \frac{n_{\text {gas }}}{m_{*}^{2}} \int \mathrm{d} \mathbf{P}^{\prime} \int \mathrm{d} \mathbf{p}^{\prime} \int \mathrm{d} \mathbf{p} \delta\left(\frac{P^{\prime 2}}{2 M}+\frac{p^{\prime 2}}{2 m}-\frac{P^{2}}{2 M}-\frac{p^{2}}{2 m}\right) \delta^{3}\left(\mathbf{P}^{\prime}+\mathbf{p}^{\prime}-\mathbf{P}-\mathbf{p}\right) \\
& \times \sigma\left(\operatorname{rel}(\mathbf{p}, \mathbf{P}), \operatorname{rel}\left(\mathbf{p}^{\prime}, \mathbf{P}^{\prime}\right)\right)\left[\mu\left(\mathbf{p}^{\prime}\right) f\left(\mathbf{P}^{\prime}\right)-\mu(\mathbf{p}) f(\mathbf{P})\right],
\end{aligned}
$$

which can be brought into the form Eq. A.1). Here the dynamics determined by collisions between test particle and gas particles is put into evidence. The collisions are characterized by a differential scattering cross-section $\sigma\left(\mathbf{p}_{f}, \mathbf{p}_{i}\right)$, together with the constraints of energy and momentum conservation expressed by the $\delta$-functions.

Exploiting the momentum conservation and introducing the momentum transfer $\mathbf{Q}=\mathbf{P}^{\prime}-\mathbf{P}$, which corresponds to the momentum gained by the test particle in a collision, we obtain

$$
\begin{aligned}
\partial_{t}^{\text {coll }} f(\mathbf{P})= & \frac{n_{\text {gas }}}{m_{*}^{2}} \int \mathrm{d} \mathbf{Q} \int \mathrm{d} \mathbf{p} \delta\left(\frac{(\mathbf{P}+\mathbf{Q})^{2}}{2 M}+\frac{(\mathbf{p}-\mathbf{Q})^{2}}{2 m}-\frac{P^{2}}{2 M}-\frac{p^{2}}{2 m}\right) \\
& \times \sigma(\operatorname{rel}(\mathbf{p}, \mathbf{P}), \operatorname{rel}(\mathbf{p}-\mathbf{Q}, \mathbf{P}+\mathbf{Q}))[\mu(\mathbf{p}-\mathbf{Q}) f(\mathbf{P}+\mathbf{Q})-\mu(\mathbf{p}) f(\mathbf{P})] .
\end{aligned}
$$

It is now convenient to express the $\delta$-function of energy conservation in terms of the momentum transfer and of the momentum components parallel and perpendicular to it. Introducing $\mathbf{P}_{\| \mathbf{Q}}=(\mathbf{P} \cdot \mathbf{Q}) \mathbf{Q} / Q^{2}$ and $\mathbf{P}_{\perp \mathbf{Q}}=\mathbf{P}-\mathbf{P}_{\| \mathbf{Q}}$ and further exploiting the definition of relative momenta given in Eq. (2.4) one has

$$
\delta\left(\frac{(\mathbf{P}+\mathbf{Q})^{2}}{2 M}+\frac{(\mathbf{p}-\mathbf{Q})^{2}}{2 m}-\frac{P^{2}}{2 M}-\frac{p^{2}}{2 m}\right)=\delta\left(\frac{Q^{2}}{2 m_{*}}-\frac{1}{m_{*}} \mathbf{Q} \cdot \operatorname{rel}\left(\mathbf{p}_{\| \mathbf{Q}}, \mathbf{P}_{\| \mathbf{Q}}\right)\right),
$$

which implies

$$
\operatorname{rel}\left(\mathbf{p}_{\| \mathbf{Q}}, \mathbf{P}_{\| \mathbf{Q}}\right)=\frac{\mathbf{Q}}{2}
$$

Since the reduced mass $m_{*}$ obeys $m_{*} / m+m_{*} / M=1$, one has the useful relation

$$
\operatorname{rel}(\mathbf{p}, \mathbf{P})+\mathbf{Q}=\operatorname{rel}(\mathbf{p}+\mathbf{Q}, \mathbf{P}-\mathbf{Q}),
$$

which, together with Eq. A.5, leads to

$$
\begin{aligned}
\sigma(\operatorname{rel}(\mathbf{p}, \mathbf{P}), \operatorname{rel}(\mathbf{p}, \mathbf{P})-\mathbf{Q})= & \sigma\left(\operatorname{rel}\left(\mathbf{p}_{\| \mathbf{Q}}, \mathbf{P}_{\| \mathbf{Q}}\right)+\operatorname{rel}\left(\mathbf{p}_{\perp \mathbf{Q}}, \mathbf{P}_{\perp \mathbf{Q}}\right)-\mathbf{Q}\right. \\
& \left.\operatorname{rel}\left(\mathbf{p}_{\| \mathbf{Q}}, \mathbf{P}_{\| \mathbf{Q}}\right)+\operatorname{rel}\left(\mathbf{p}_{\perp \mathbf{Q}}, \mathbf{P}_{\perp \mathbf{Q}}\right)\right) \\
= & \sigma\left(\operatorname{rel}\left(\mathbf{p}_{\perp \mathbf{Q}}, \mathbf{P}_{\perp \mathbf{Q}}\right)-\frac{1}{2} \mathbf{Q}, \operatorname{rel}\left(\mathbf{p}_{\perp \mathbf{Q}}, \mathbf{P}_{\perp \mathbf{Q}}\right)+\frac{1}{2} \mathbf{Q}\right) .
\end{aligned}
$$

Eq. A.3 now becomes

$$
\begin{aligned}
\partial_{t}^{\text {coll }} f(\mathbf{P})= & \frac{n_{\text {gas }}}{m_{*}^{2}} \int \mathrm{d} \mathbf{Q} \int \mathrm{d} \mathbf{p} \sigma\left(\operatorname{rel}\left(\mathbf{p}_{\perp}, \mathbf{P}_{\perp \mathbf{Q}}\right)-\frac{1}{2} \mathbf{Q}, \operatorname{rel}\left(\mathbf{p}_{\perp}, \mathbf{P}_{\perp \mathbf{Q}}\right)+\frac{1}{2} \mathbf{Q}\right) \\
& \times \delta\left(\frac{Q^{2}}{2 m_{*}}-\frac{1}{m_{*}} \mathbf{Q} \cdot \operatorname{rel}\left(\mathbf{p}_{\| \mathbf{Q}}, \mathbf{P}_{\| \mathbf{Q}}\right)\right)[\mu(\mathbf{p}-\mathbf{Q}) f(\mathbf{P}+\mathbf{Q})-\mu(\mathbf{p}) f(\mathbf{P})] .
\end{aligned}
$$


By means of the shift of coordinates

$$
\mathbf{p} \rightarrow \mathbf{p}+\frac{m}{m_{*}} \frac{\mathbf{Q}}{2}+\frac{m}{M} \mathbf{P}_{\| \mathbf{Q}},
$$

which leaves $\mathbf{p}_{\perp}$ invariant, the argument of the delta function simplifies to $\mathbf{Q} \cdot \mathbf{p}$, such that it can be evaluated explicitly leading to

$$
\begin{aligned}
\partial_{t}^{\text {coll }} f(\mathbf{P})= & \frac{n_{\text {gas }} m}{m_{*}^{2}} \int \frac{\mathrm{d} \mathbf{Q}}{Q} \int_{\mathbf{Q}^{\perp}} \mathrm{d} \mathbf{k}_{\perp} \sigma\left(\operatorname{rel}\left(\mathbf{k}_{\perp}, \mathbf{P}_{\perp \mathbf{Q}}\right)-\frac{\mathbf{Q}}{2}, \operatorname{rel}\left(\mathbf{k}_{\perp}, \mathbf{P}_{\perp \mathbf{Q}}\right)+\frac{\mathbf{Q}}{2}\right) \\
& \times\left[\mu\left(\mathbf{k}_{\perp}+\frac{m}{m_{*}} \frac{\mathbf{Q}}{2}+\frac{m}{M}\left(\mathbf{P}_{\| \mathbf{Q}}-\mathbf{Q}\right)\right) f(\mathbf{P}-\mathbf{Q})-\mu\left(\mathbf{k}_{\perp}+\frac{m}{m_{*}} \frac{\mathbf{Q}}{2}+\frac{m}{M} \mathbf{P}_{\| \mathbf{Q}}\right) f(\mathbf{P})\right] .
\end{aligned}
$$

This is the explicit expression allowing one to straightforwardly see the connection between the classical and the quantum linear Boltzmann equation. Another useful form is given by Eq. A.14).

\section{A.2. Approach to equilibrium}

We now prove that the relative entropy of a solution of the classical linear Boltzmann equation with respect to its stationary solution is a monotonically decreasing function, equal to zero if and only if the solution is at equilibrium. Let us first consider the explicit derivative of the relative entropy (4.16),

$$
\frac{\mathrm{d} H\left(f \mid f_{\mathrm{EQ}}\right)}{\mathrm{d} t}=\int \mathrm{d} \mathbf{P} \partial_{t}^{\text {coll }} f(\mathbf{P})\left[1+\log \frac{f(\mathbf{P})}{f_{\mathrm{EQ}}(\mathbf{P})}\right] \text {. }
$$

Exploiting the relations Eq. (2.12) and Eq. (2.11) for the loss and gain rates, the classical linear Boltzmann equation (3.3) reads

$$
\partial_{t}^{\text {coll }} f(\mathbf{P})=\int \mathrm{d} \mathbf{P}^{\prime}\left[M^{\mathrm{cl}}\left(\mathbf{P}^{\prime} \rightarrow \mathbf{P}\right) f\left(\mathbf{P}^{\prime}\right)-M^{\mathrm{cl}}\left(\mathbf{P} \rightarrow \mathbf{P}^{\prime}\right) f(\mathbf{P})\right] .
$$

The fact that the transition rates obey the detailed balance condition Eq. (4.9) implies that the quantity

$$
W\left(\mathbf{P}, \mathbf{P}^{\prime}\right)=M^{\mathrm{cl}}\left(\mathbf{P}^{\prime} \rightarrow \mathbf{P}\right) \mathrm{e}^{\beta \frac{P^{2}}{2 M}}
$$

is symmetric in its arguments. We thus obtain an expression

$$
\partial_{t}^{\text {coll }} f(\mathbf{P})=\int \mathrm{d} \mathbf{P}^{\prime} W\left(\mathbf{P}, \mathbf{P}^{\prime}\right)\left[f_{\mathrm{EQ}}(\mathbf{P}) f\left(\mathbf{P}^{\prime}\right)-f_{\mathrm{EQ}}\left(\mathbf{P}^{\prime}\right) f(\mathbf{P})\right],
$$

that puts into evidence that the integrand is a product of functions which are symmetric and antisymmetric under the exchange of $\mathbf{P}$ and $\mathbf{P}^{\prime}$. Using this form, the time derivative of the relative entropy therefore reads as

$$
\begin{aligned}
\frac{\mathrm{d} H\left(f \mid f_{\mathrm{EQ}}\right)}{\mathrm{d} t}= & \frac{1}{2} \int \mathrm{d} \mathbf{P} \int \mathrm{d} \mathbf{P}^{\prime} W\left(\mathbf{P}^{\prime} \rightarrow \mathbf{P}\right)\left[f_{\mathrm{EQ}}(\mathbf{P}) f\left(\mathbf{P}^{\prime}\right)-f_{\mathrm{EQ}}\left(\mathbf{P}^{\prime}\right) f(\mathbf{P})\right] \\
& \times\left[\log \left(f_{\mathrm{EQ}}\left(\mathbf{P}^{\prime}\right) f(\mathbf{P})\right)-\log \left(f_{\mathrm{EQ}}(\mathbf{P}) f\left(\mathbf{P}^{\prime}\right)\right)\right] .
\end{aligned}
$$

By noting that

$$
(X-Y)(\log Y-\log X) \leqslant 0,
$$

where the equal sign holds if and only if $X=Y$, this proves that the time derivative of the relative entropy is equal to zero if and only if one considers the equilibrium solution.

Moreover, since $\partial_{t}^{\text {coll }} f(\mathbf{P})=0$ implies $\mathrm{d} H\left(f \mid f_{\mathrm{EQ}}\right) / \mathrm{d} t=0$, it follows that $\mathrm{d} H\left(f \mid f_{\mathrm{EQ}}\right) / \mathrm{d} t=0$, or equivalently $f=f_{\mathrm{EQ}}$, is a necessary condition for a stationary solution. But we already know that $f=f_{\mathrm{EQ}}$ is a sufficient condition for stationarity, such that the stationary solution is unique. 


\section{A.3. The friction coefficient of classical Brownian motion}

We now explicitly derive the microscopic expression for the friction coefficient appearing in the classical Kramers equation (5.1). Let us start from the classical linear Boltzmann equation written as in Eq. (A.1), with the constraint $|\operatorname{rel}(\mathbf{p}, \mathbf{P})|=\left|\operatorname{rel}\left(\mathbf{p}^{\prime}, \mathbf{P}^{\prime}\right)\right|$ keeping track of energy conservation in each collision. We put $f(\mathbf{P})=\nu_{\mathrm{EQ}}(\mathbf{P}) \chi(\mathbf{P})$, where $\nu_{\mathrm{EQ}}(\mathbf{P})$ has the form Eq. (4.7) of a Maxwell-Boltzmann distribution of the test particle. Exploiting energy conservation Eq. (A.1) now takes the more explicit form

$$
\begin{aligned}
\partial_{t} f(\mathbf{P})= & \frac{n_{\text {gas }}}{m_{*}} \nu_{\mathrm{EQ}}(\mathbf{P}) \int_{0}^{\infty} \mathrm{d} p p^{2} \mu_{\beta}(\mathbf{p}) \int_{0}^{2 \pi} \mathrm{d} \Phi \int_{0}^{\pi} \mathrm{d} \Theta \sin \Theta \int_{0}^{2 \pi} \mathrm{d} \varphi \int_{0}^{\pi} \mathrm{d} \vartheta \sin \vartheta \\
& \times|\operatorname{rel}(\mathbf{p}, \mathbf{P})| \sigma(\vartheta ;|\operatorname{rel}(\mathbf{p}, \mathbf{P})|)\left[\chi\left(\mathbf{P}^{\prime}\right)-\chi(\mathbf{P})\right],
\end{aligned}
$$

where $(\vartheta, \varphi)$ denote the polar angles of $\operatorname{rel}\left(\mathbf{p}^{\prime}, \mathbf{P}^{\prime}\right)$ with respect to $\operatorname{rel}(\mathbf{p}, \mathbf{P})$, and $(\Theta, \Phi)$ the polar angles of $\mathbf{p}$ with respect to $\mathbf{P}$.

As discussed in Sect. 5 in the Brownian motion limit one considers a test particle much heavier than the gas particles, such that $m / M \ll 1$, and the test particle is assumed to be close to thermal equilibrium. Under these assumptions the momentum transfer in a single collision, given by

$$
\mathbf{Q}=\mathbf{P}^{\prime}-\mathbf{P}=-\left[\operatorname{rel}\left(\mathbf{p}^{\prime}, \mathbf{P}^{\prime}\right)-\operatorname{rel}(\mathbf{p}, \mathbf{P})\right],
$$

can be considered small. We therefore expand the function $\chi\left(\mathbf{P}^{\prime}\right)$ in a Taylor series, thus obtaining up to second order

$$
\begin{aligned}
\partial_{t} f(\mathbf{P})= & \frac{n_{\text {gas }}}{m_{*}} \nu_{\mathrm{EQ}}(\mathbf{P}) \int_{0}^{\infty} \mathrm{d} p p^{2} \mu_{\beta}(\mathbf{p}) \int_{0}^{2 \pi} \mathrm{d} \Phi \int_{0}^{\pi} \mathrm{d} \Theta \sin \Theta \int_{0}^{2 \pi} \mathrm{d} \varphi \int_{0}^{\pi} \mathrm{d} \vartheta \sin \vartheta \\
& \times|\operatorname{rel}(\mathbf{p}, \mathbf{P})| \sigma(\vartheta ;|\operatorname{rel}(\mathbf{p}, \mathbf{P})|) \\
& \times\left[\sum_{i=1}^{3}\left(\mathbf{P}^{\prime}-\mathbf{P}\right)_{i} \frac{\partial}{\partial P_{i}} \chi(\mathbf{P})+\frac{1}{2} \sum_{i, j=1}^{3}\left(\mathbf{P}^{\prime}-\mathbf{P}\right)_{j}\left(\mathbf{P}^{\prime}-\mathbf{P}\right)_{i} \frac{\partial^{2}}{\partial P_{j} \partial P_{i}} \chi(\mathbf{P})\right] .
\end{aligned}
$$

Thanks to the identity Eq. A.18) and to the fact that the scattering cross-section only depends on the angles $\vartheta$ and $\Theta$, one can perform the integration over the azimuthal angle $\varphi$ appearing only in $\mathbf{P}^{\prime}-\mathbf{P}$. It is done by exploiting the integrals

$$
\begin{aligned}
\int_{0}^{2 \pi} \mathrm{d} \varphi\left(\mathbf{K}^{\prime}-\mathbf{K}\right)_{i} & =2 \pi\left(\frac{K^{\prime}}{K} \cos \vartheta-1\right) K_{i} \\
\int_{0}^{2 \pi} \mathrm{d} \varphi\left(\mathbf{K}^{\prime}-\mathbf{K}\right)_{j}\left(\mathbf{K}^{\prime}-\mathbf{K}\right)_{i} & =2 \pi\left[\frac{1}{2} \sin ^{2} \vartheta K^{2} \delta_{i j}-\frac{1}{2}(3 \cos \vartheta-1)(1-\cos \vartheta) K_{j} K_{i}\right],
\end{aligned}
$$

which are valid whenever $(\vartheta, \varphi)$ denote the polar angles of $\mathbf{K}^{\prime}$ with respect to $\mathbf{K}$.

To obtain Eq. A.20 let us denote by $\mathbf{e}_{1}, \mathbf{e}_{2}$, and $\mathbf{e}_{3}$ the unit basis vectors for the Cartesian coordinates, such that $K_{i}=\mathbf{e}_{i} \cdot \mathbf{K}$, and consider another set of basis vectors $\mathbf{e}_{1}^{\prime}, \mathbf{e}_{2}^{\prime}$ and $\mathbf{e}_{3}^{\prime}$ such that $\mathbf{e}_{3}^{\prime}$ is in the direction of $\mathbf{K}$. One then has

$$
\begin{aligned}
\left(\mathbf{K}^{\prime}-\mathbf{K}\right)_{i} & =\mathbf{e}_{i} \cdot\left(\mathbf{K}^{\prime}-\mathbf{K}\right) \\
& =\mathbf{e}_{i} \cdot\left[K^{\prime} \sin \vartheta \cos \varphi \mathbf{e}_{1}^{\prime}+K^{\prime} \sin \vartheta \sin \varphi \mathbf{e}_{2}^{\prime}+\left(K^{\prime} \cos \vartheta-K\right) \mathbf{e}_{3}^{\prime}\right] .
\end{aligned}
$$

Equation A.20 is obtained by integrating the azimuthal angle $\varphi$ and recalling $\mathbf{K}=K \mathbf{e}_{3}^{\prime}$. A similar, but much longer calculation leads to Eq. A.21).

We are left with contributions in the integrand proportional to $\operatorname{rel}(\mathbf{p}, \mathbf{P})_{i}$ and $\operatorname{rel}(\mathbf{p}, \mathbf{P})_{j} \operatorname{rel}(\mathbf{p}, \mathbf{P})_{i}$, such that the integrals A.20 and A.21 can be applied once more to perform the integrations over $\Phi$. The result of the integration over the azimuthal angles reads 


$$
\begin{aligned}
\partial_{t} f(\mathbf{P})= & 4 \pi^{2} \frac{n_{\text {gas }}}{m_{*}} \nu_{\mathrm{EQ}}(\mathbf{P}) \int_{0}^{\infty} \mathrm{d} p p^{2} \mu_{\beta}(\mathbf{p}) \int_{0}^{\pi} \mathrm{d} \Theta \sin \Theta \int_{0}^{\pi} \mathrm{d} \vartheta \sin \vartheta(1-\cos \vartheta)|\operatorname{rel}(\mathbf{p}, \mathbf{P})| \\
& \times \sigma(\vartheta ;|\operatorname{rel}(\mathbf{p}, \mathbf{P})|)\left\{\left(\frac{M}{m} \frac{p}{P} \cos \Theta-1\right) \frac{m_{*}}{M} \sum_{i=1}^{3} P_{i} \frac{\partial}{\partial P_{i}} \chi(\mathbf{P})\right. \\
& +\frac{1}{4}\left[(1+\cos \vartheta)|\operatorname{rel}(\mathbf{p}, \mathbf{P})|^{2}+\frac{1}{2}(1-3 \cos \vartheta) \frac{m_{*}^{2}}{m^{2}} p^{2} \sin ^{2} \Theta\right] \sum_{i=1}^{3} \frac{\partial^{2}}{\partial P_{i}^{2}} \chi(\mathbf{P}) \\
& \left.+\frac{1}{4}(1-3 \cos \vartheta)\left[\left(\frac{M}{m} \frac{p}{P} \cos \Theta-1\right)^{2}-\frac{1}{2}\left(\frac{M}{m} \frac{p}{P} \sin \Theta\right)^{2}\right] \frac{m_{*}^{2}}{M^{2}} \sum_{i, j=1}^{3} P_{j} P_{i} \frac{\partial^{2}}{\partial P_{j} \partial P_{i}} \chi(\mathbf{P})\right\} .
\end{aligned}
$$

To proceed we exploit the fact that the test particle is assumed to be close to equilibrium, such that $p / P \approx \sqrt{m / M}$, and $m / M \ll 1$. We expand the expression of $|\operatorname{rel}(\mathbf{p}, \mathbf{P})|$ in this small ratio, thus obtaining up to order $\sqrt{m / M}$

$$
|\operatorname{rel}(\mathbf{p}, \mathbf{P})| \approx p\left[1-\frac{m}{M} \frac{P}{p} \cos \Theta\right]
$$

and therefore in particular

$$
|\operatorname{rel}(\mathbf{p}, \mathbf{P})| \sigma(\vartheta ;|\operatorname{rel}(\mathbf{p}, \mathbf{P})|) \approx p\left[\sigma(\vartheta ; p)-\frac{m}{M} \frac{P}{p} \cos \Theta\left(\sigma(\vartheta ; p)+p \frac{\partial}{\partial p} \sigma(\vartheta ; p)\right)\right] .
$$

As a result of this approximation one can perform the integral over $\Theta$, without explicit knowledge of the scattering cross-section, which only depends on $p$ and $\vartheta$ for an isotropic interaction potential. Inserting Eq. A.25) and Eq. A.24 in Eq. A.23 and performing the angular integral one obtains after lengthy but straightforward calculations

$$
\begin{aligned}
\partial_{t} f(\mathbf{P})= & -\frac{8}{3} \pi^{2} \frac{n_{\text {gas }}}{m} \nu_{\mathrm{EQ}}(\mathbf{P}) \frac{\beta}{M} \int_{0}^{\infty} \mathrm{d} p p^{5} \mu_{\beta}(\mathbf{p}) \int_{0}^{\pi} \mathrm{d} \vartheta \sin \vartheta(1-\cos \vartheta) \sigma(\vartheta ; p) \\
& \times \sum_{i=1}^{3}\left[P_{i} \frac{\partial}{\partial P_{i}} \chi(\mathbf{P})-\frac{M}{\beta} \frac{\partial^{2}}{\partial P_{i}^{2}} \chi(\mathbf{P})\right] .
\end{aligned}
$$

Here, an integration by parts has been exploited,

$$
\int_{0}^{\infty} \mathrm{d} p p^{4} \mu_{\beta}(\mathbf{p}) \frac{\partial}{\partial p} \sigma(\vartheta ; p)=-\int_{0}^{\infty} \mathrm{d} p\left(4 p^{3}-\frac{2}{p_{\beta}^{2}} p^{5}\right) \mu_{\beta}(\mathbf{p}) \sigma(\vartheta ; p) .
$$

As a last step, we return to an equation for the original distribution $f(\mathbf{P})=\nu_{\mathrm{EQ}}(\mathbf{P}) \chi(\mathbf{P})$. We recall that the equilibrium solution $\nu_{\mathrm{EQ}}(\mathbf{P})$, as defined in Eq. (4.7), is a null eigenvector of the Fokker-Planck operator

$$
D[g(\mathbf{P})]=\sum_{i=1}^{3}\left[\frac{\partial}{\partial P_{i}}\left(P_{i} g(\mathbf{P})\right)+\frac{M}{\beta} \frac{\partial^{2}}{\partial P_{i}^{2}} g(\mathbf{P})\right],
$$

such that in particular

$$
D\left[\nu_{\mathrm{EQ}}(\mathbf{P}) \chi(\mathbf{P})\right]=-\nu_{\mathrm{EQ}}(\mathbf{P}) \sum_{i=1}^{3}\left[P_{i} \frac{\partial}{\partial P_{i}} \chi(\mathbf{P})-\frac{M}{\beta} \frac{\partial^{2}}{\partial P_{i}^{2}} \chi(\mathbf{P})\right] .
$$

We are thus left with 


$$
\begin{aligned}
\partial_{t} f(\mathbf{P})= & \frac{8}{3} \pi^{2} \frac{n_{\text {gas }}}{m} \frac{\beta}{M} \int_{0}^{\infty} \mathrm{d} p p^{5} \mu_{\beta}(\mathbf{p}) \int_{0}^{\pi} \mathrm{d} \vartheta \sin \vartheta(1-\cos \vartheta) \sigma(\vartheta ; p) \\
& \times \sum_{i=1}^{3}\left[\frac{\partial}{\partial P_{i}}\left(P_{i} f(\mathbf{P})\right)+\frac{M}{\beta} \frac{\partial^{2}}{\partial P_{i}^{2}} f(\mathbf{P})\right] .
\end{aligned}
$$

Switching to dimensionless variables $u=p / p_{\beta}$ and using Eq. (2.6) for $\mu_{\beta}$ (p), one finally has

$$
\partial_{t} f(\mathbf{P})=\eta \sum_{i=1}^{3}\left[\frac{\partial}{\partial P_{i}}\left(P_{i} f(\mathbf{P})\right)+\frac{M}{\beta} \frac{\partial^{2}}{\partial P_{i}^{2}} f(\mathbf{P})\right]
$$

with

$$
\eta=\frac{16}{3} \sqrt{\pi} \frac{m}{M} n_{\text {gas }} \sqrt{\frac{2}{\beta m}} \int_{0}^{\infty} \mathrm{d} u u^{5} \mathrm{e}^{-u^{2}} \int_{0}^{\pi} \mathrm{d} \vartheta \sin \vartheta(1-\cos \vartheta) \sigma\left(\vartheta ; u p_{\beta}\right),
$$

as in Eq. (5.2).

\section{Appendix B. List of symbols}

$$
\begin{aligned}
& \beta \quad \text { inverse temperature, } \beta=1 /\left(k_{\mathrm{B}} T\right) \\
& D_{p p} \quad \text { diffusion coefficient, see (5.16) } \\
& D_{x x} \quad \text { diffusion coefficient, see (5.17) } \\
& \eta \quad \text { friction coefficient, see 4.33), (5.13) } \\
& \operatorname{erf}(x) \quad \text { error function } 198 \\
& E(\mathbf{Q}, \mathbf{P}) \quad \text { energy transfer to test particle, see 4.11) } \\
& \Phi_{\mathcal{P}} \quad \text { characteristic function of momentum transfer distribution, see (7.12), (7.15) } \\
& f(\mathbf{P}) \quad \text { distribution function of the test particle momentum } \\
& f\left(\mathbf{p}_{f}, \mathbf{p}_{i}\right) \quad \text { elastic scattering amplitude } \\
& f_{0}(\mathbf{p}) \quad \text { forward scattering amplitude, } f_{0}(\mathbf{p})=f(\mathbf{p}, \mathbf{p}) \\
& f_{B}(\mathbf{Q}) \quad \text { scattering amplitude in Born approximation, see 2.15) } \\
& { }_{1} F_{1} \quad \text { confluent hypergeometric function [198] } \\
& \Gamma(x) \quad \text { gamma function } 198 \\
& \Gamma_{\text {tot }} \quad \text { total scattering rate, see (7.4) } \\
& \Gamma_{\beta} \quad \text { thermal scattering rate, see 44.28) } \\
& \tilde{\Gamma}(U) \quad \text { dimensionless loss rate, see (4.54) } \\
& \mathrm{H}_{0} \quad \text { kinetic energy operator of test particle, } \mathrm{H}_{0}=\mathrm{P}^{2} /(2 M) \\
& H_{\mathrm{n}} \quad \text { gas induced energy shift, see (2.5), (6.2) } \\
& \text { K scaled momentum transfer, see (4.52) } \\
& \lambda_{\text {th }} \quad \text { thermal de Broglie wave length of test particle, } \lambda_{\text {th }}=\sqrt{2 \pi \hbar^{2} \beta / M} \\
& L(\mathbf{p}, \mathbf{P} ; \mathbf{Q}) \quad \text { non-unitary part of Lindblad operator, see (2.3) }
\end{aligned}
$$




\begin{tabular}{|c|c|}
\hline$L_{B}(\mathbf{P} ; \mathbf{Q})$ & non-unitary part of Lindblad operator in Born approximation, see (2.17) \\
\hline $\mathcal{L}$ & superoperator of the dissipative part of the quantum linear Boltzmann equation, see (2.1) \\
\hline$\mu(\mathbf{p})$ & distribution function of gas momenta \\
\hline$\mu_{\beta}(\mathbf{p})$ & Maxwell-Boltzmann distribution, see (2.6); for $\mu_{\beta}^{(1 d, 2 d)}$ see (8.2), (8.3) \\
\hline$m$ & mass of gas particle \\
\hline$m_{*}$ & reduced mass, $m_{*}=m M /(m+M)$ \\
\hline$M$ & mass of test particle \\
\hline$M_{\mathrm{in}}^{\mathrm{cl}}(\mathbf{P} ; \mathbf{Q})$ & classical gain rate, see (2.11) \\
\hline$M_{\text {out }}^{\mathrm{cl}}(\mathbf{P})$ & classical loss rate, see (2.12) \\
\hline $\mathcal{M}$ & superoperator of the quantum linear Boltzmann equation, see (2.1) \\
\hline$n_{\text {gas }}$ & density of gas particles \\
\hline$n \equiv n_{1}+i n_{2}$ & index of refraction, see (6.14) \\
\hline$\nu_{\mathrm{EQ}}(\mathbf{P})$ & Maxwell-Boltzmann distribution for the test particle, see (4.7) \\
\hline$p_{\beta}$ & most probable momentum in thermal gas distribution, $p_{\beta}=\sqrt{2 m / \beta}$ \\
\hline $\mathrm{P} \equiv\left(\mathrm{P}_{1}, \mathrm{P}_{2}, \mathrm{P}_{3}\right)$ & momentum operator of test particle \\
\hline Q & momentum transfer to test particle \\
\hline $\mathbf{Q}^{\perp}$ & set of all momenta perpendicular to $\mathbf{Q}$, i.e., $\mathbf{Q}^{\perp}=\left\{\mathbf{p} \in \mathbb{R}^{3}: \mathbf{p} \cdot \mathbf{Q}=0\right\}$ \\
\hline$\rho$ & quantum state of motion of test particle \\
\hline $\operatorname{rel}(\mathbf{p}, \mathbf{P})$ & relative momentum, see (2.4) \\
\hline$\sigma\left(\mathbf{p}_{f}, \mathbf{p}_{i}\right)$ & differential cross-section, $\sigma\left(\mathbf{p}_{f}, \mathbf{p}_{i}\right)=\left|f\left(\mathbf{p}_{f}, \mathbf{p}_{i}\right)\right|^{2}$ \\
\hline$\sigma\left(\vartheta ; p_{i}\right)$ & differential cross-section (isotropic potential), $\cos \vartheta=\mathbf{p}_{i} \cdot \mathbf{p}_{f} / p_{i}^{2}, p_{i}=\left|\mathbf{p}_{i}\right|$ \\
\hline$\sigma_{\text {tot }}$ & total elastic scattering cross-section, see (6.4), (7.20) \\
\hline$\sigma_{B}(\mathbf{Q})$ & differential cross-section in Born approximation, $\sigma_{B}(\mathbf{Q})=\left|f_{B}(\mathbf{Q})\right|^{2}$ \\
\hline$S(\mathbf{Q}, E)$ & dynamic structure factor, see (8.12) \\
\hline $\mathbf{U}$ & scaled momentum, see 4.51) \\
\hline$v_{\beta}$ & most probable velocity in thermal gas distribution, $v_{\beta}=p_{\beta} / m$ \\
\hline$V(\mathbf{X}-\mathbf{x})$ & interaction potential between test and gas particle \\
\hline$V_{\mathrm{opt}}$ & optical potential, see (6.8) \\
\hline $\mathrm{X} \equiv\left(\mathrm{X}_{1}, \mathrm{X}_{2}, \mathrm{X}_{3}\right)$ & position operator of test particle \\
\hline $\mathbf{X}_{\| \mathbf{Q}}$ & vector component parallel to $\mathbf{Q}$, i.e., $\mathbf{X}_{\| \mathbf{Q}}=(\mathbf{X} \cdot \mathbf{Q}) \mathbf{Q} / Q^{2}$ \\
\hline $\mathbf{X}_{\perp \mathbf{Q}}$ & vector component orthogonal to $\mathbf{Q}$, i.e., $\mathbf{X}_{\perp \mathbf{Q}}=\mathbf{X}-\mathbf{X}_{\| \mathbf{Q}}$ \\
\hline
\end{tabular}

References

[1] L. Boltzmann, Vorlesungen über Gastheorie, Barth, Leipzig, 1898. 
[2] C. Cercignani, Theory and application of the Boltzmann equation, Scottisch Academic Press, Edinburgh, 1975.

[3] H. Spohn, Large scale dynamics of interacting particles, Springer, Berlin, 1991.

[4] S. Harris, An Introduction to the Theory of the Boltzmann Equation, Courier Dover Publications, 2004.

[5] R. Balian, From microphysics to macrophysics: methods and applications of statistical physics. Vol. II, Springer, Berlin, 2007.

[6] B. Davison, B. Sykes, Neutron transport theory, Clarendon Press, Oxford, 1957.

[7] M. M. R. Williams, The Slowing Down and Thermalization of Neutrons, North-Holland, Amsterdam, 1966.

[8] L. W. Nordheim, On the kinetic method in the new statistics and its application in the electron theory of conductivity, Proc. Roy. Soc. 119 (1928) 689-698.

[9] E. A. Uehling, G. E. Uhlenbeck, Transport phenomena in Einstein-Bose and Fermi-Dirac gases. I, Phys. Rev. 43 (1933) $552-561$.

[10] J. Ross, J. G. Kirkwood, The statistical-mechanical theory of transport processes. VIII. Quantum theory of transport in gases, J. Chem. Phys. 22 (1954) 1094-1103.

[11] C. W. Gardiner, P. Zoller, Quantum kinetic theory: A quantum kinetic master equation for condensation of a weakly interacting Bose gas without a trapping potential, Phys. Rev. A 55 (1997) 2902-2921.

[12] D. Jaksch, C. W. Gardiner, P. Zoller, Quantum kinetic theory. II. Simulation of the quantum Boltzmann master equation, Phys. Rev. A 56 (1997) 575-586.

[13] C. W. Gardiner, P. Zoller, R. J. Ballagh, M. J. Davis, Kinetics of Bose-Einstein condensation in a trap, Phys. Rev. Lett. 79 (1997) 1793-1796.

[14] C. W. Gardiner, P. Zoller, Quantum kinetic theory. III. Quantum kinetic master equation for strongly condensed trapped systems, Phys. Rev. A 58 (1998) 536-556.

[15] D. Jaksch, C. W. Gardiner, K. M. Gheri, P. Zoller, Quantum kinetic theory. IV. Intensity and amplitude fluctuations of a Bose-Einstein condensate at finite temperature including trap loss, Phys. Rev. A 58 (1998) 1450-1464.

[16] C. W. Gardiner, M. D. Lee, R. J. Ballagh, M. J. Davis, P. Zoller, Quantum kinetic theory of condensate growth: Comparison of experiment and theory, Phys. Rev. Lett. 81 (1998) 5266-5269.

[17] C. W. Gardiner, P. Zoller, Quantum kinetic theory. V. Quantum kinetic master equation for mutual interaction of condensate and noncondensate, Phys. Rev. A 61 (2000) 033601.

[18] H. Spohn, Kinetic equations from Hamiltonian dynamics: Markovian limits, Rev. Mod. Phys. 52 (1980) 569-615.

[19] R. F. Snider, Relaxation and transport of molecular systems in the gas phase, Int. Rev. Phys. Chem. 17 (1998) $185-225$.

[20] L. Erds, M. Salmhofer, H. Yau, On the quantum Boltzmann equation, J. Stat. Phys. 116 (2004) 367-380.

[21] D. Benedetto, F. Castella, R. Esposito, M. Pulvirenti, A short review on the derivation of the nonlinear quantum Boltzmann equations, Commun. Math. Sci. (2007) 55-71.

[22] J. Lukkarinen, H. Spohn, Not to normal order - notes on the kinetic limit for weakly interacting quantum fluids, J. Stat. Phys. 134 (2009) 1133-1172.

[23] H. Spohn, Kinetic equations for quantum many-particle systems, in: Modern Encyclopedia of Mathematical Physics, Springer, to appear, preprint arXiv:0706.0807

[24] F. Castella, From the von-Neumann equation to the quantum Boltzmann equation in a deterministic framework, J. Stat. Phys. 104 (2001) 387-447.

[25] F. Castella, From the von Neumann equation to the quantum Boltzmann equation II: identifying the Born series, J. Stat. Phys. 106 (2002) 1197-1220.

[26] D. Eng, L. Erds, The linear Boltzmann equation as the low density limit of a random Schrödinger equation, Rev. Math. Phys. 17 (2005) 669-743.

[27] R. Alicki, K. Lendi, Quantum Dynamical Semigroups and Applications, 2nd Edition, Vol. 717 of Lecture Notes in Physics, Springer, Berlin, 2007.

[28] A. S. Holevo, Statistical Structure of Quantum Theory, Vol. m 67 of Lecture Notes in Physics, Springer, Berlin, 2001.

[29] H.-P. Breuer, F. Petruccione, The Theory of Open Quantum Systems, Oxford University Press, Oxford, 2007.

[30] V. Gorini, A. Kossakowski, E. C. G. Sudarshan, Completely positive dynamical semigroups of $N$-level systems, J. Math. Phys. 17 (1976) 821-825.

[31] G. Lindblad, On the generators of quantum dynamical semigroups, Comm. Math. Phys. 48 (1976) 119-130.

[32] K. Hornberger, B. Vacchini, Monitoring derivation of the quantum linear Boltzmann equation, Phys. Rev. A 77 (2008) 022112 .

[33] A. S. Holevo, Covariant quantum dynamical semigroups: unbounded generators, in: A. Böhm, H. D. Doebner, P. Kielanowski (Eds.), Irreversibility and causality, Vol. 504 of Lecture Notes in Physics, Springer, Berlin, 1998, pp. $67-81$.

[34] M. Arndt, K. Hornberger, A. Zeilinger, Probing the limits of the quantum world, Physics World 18 (2005) 35-40.

[35] A. Cronin, J. Schmiedmayer, D. Pritchard, Optics and interferometry with atoms and molecules, Rev. Mod. Phys. (2009) in press eprint arXiv: 0712.3703.

[36] S. Gerlich, L. Hackermüller, K. Hornberger, A. Stibor, H. Ulbricht, M. Gring, F. Goldfarb, T. Savas, M. Müri, M. Mayor, M. Arndt, A Kapitza-Dirac-Talbot-Lau interferometer for highly polarizable molecules, Nature Phys. 3 (2007) 711-715.

[37] J. Schmiedmayer, M. S. Chapman, C. R. Ekstrom, T. D. Hammond, S. Wehinger, D. E. Pritchard, Index of refraction of various gases for sodium matter waves, Phys. Rev. Lett. 74 (1995) 1043-1047.

[38] M. Jacquey, M. Buchner, G. Trenec, J. Vigue, First measurements of the index of refraction of gases for lithium atomic waves, Phys. Rev. Lett. 98 (2007) 240405. 
[39] K. Hornberger, S. Uttenthaler, B. Brezger, L. Hackermüller, M. Arndt, A. Zeilinger, Collisional decoherence observed in matter wave interferometry, Phys. Rev. Lett. 90 (2003) 160401.

[40] E. Joos, H. D. Zeh, The emergence of classical properties through interaction with the environment, Z. Phys. B: Condens. Matter 59 (1985) 223-243.

[41] K. Hornberger, Master equation for a quantum particle in a gas, Phys. Rev. Lett. 97 (2006) 060601.

[42] B. Vacchini, Test particle in a quantum gas, Phys. Rev. E 63 (2001) 066115.

[43] M. R. Gallis, G. N. Fleming, Environmental and spontaneous localization, Phys. Rev. A 42 (1990) 38-48.

[44] L. Diósi, Quantum master equation of a particle in a gas environment, Europhys. Lett. 30 (1995) 63-68.

[45] L. Lanz, B. Vacchini, Incoherent dynamics in neutron-matter interaction, Phys. Rev. A 56 (1997) 4826-4838.

[46] B. Vacchini, Completely positive quantum dissipation, Phys. Rev. Lett. 84 (2000) 1374-1377.

[47] B. Vacchini, Translation-covariant Markovian master equation for a test particle in a quantum fluid, J. Math. Phys. 42 (2001) 4291-4312.

[48] R. Alicki, Search for a border between classical and quantum worlds, Phys. Rev. A 65 (2002) 034104.

[49] P. J. Dodd, J. J. Halliwell, Decoherence and records for the case of a scattering environment, Phys. Rev. D 67 (2003) 105018.

[50] J. Clark, Decoherence rates for Galilean covariant dynamics, J. Math. Phys. 49 (2008) 052103.

[51] J. Clark, An infinite-temperature limit for a quantum scattering process, Rep. Math. Phys. 63 (2009) 131-152.

[52] J. Clark, The reduced effect of a single scattering with a low-mass particle via a point interaction, J. Funct. Anal. 256 (2009) 2894-2916.

[53] J. Clark, W. De Roeck, C. Maes, Diffusive behavior from a quantum master equation, eprint arXiv:0812.2858v1.

[54] K. Hornberger, J. E. Sipe, Collisional decoherence reexamined, Phys. Rev. A 68 (2003) 012105.

[55] L. Hackermüller, K. Hornberger, B. Brezger, A. Zeilinger, M. Arndt, Decoherence in a Talbot Lau interferometer: The influence of molecular scattering, Appl. Phys. B 77 (2003) 781-787.

[56] F. Petruccione, B. Vacchini, Quantum description of Einstein's Brownian motion, Phys. Rev. E 71 (2005) 046134.

[57] L. Lanz, B. Vacchini, Dynamical semigroup description of coherent and incoherent particle-matter interaction, Int. J. Theor. Phys. 36 (1997) 67-88.

[58] T. P. Altenmüller, R. Müller, A. Schenzle, Atom-interferomeric study of Bose-Einstein condensation, Phys. Rev. A 56 (1997) 2959-2971.

[59] A. Teta, On a rigorous proof of the Joos-Zeh formula for decoherence in a two-body problem, in: Multiscale methods in quantum mechanics, Trends Math., Birkhäuser, Boston, 2004, pp. 197-205.

[60] D. Dürr, R. Figari, A. Teta, Decoherence in a two-particle model, J. Math. Phys. 45 (2004) 1291-1309.

[61] R. Adami, R. Figari, D. Finco, A. Teta, On the asymptotic behaviour of a quantum two-body system in the small mass ratio limit, J. Phys. A: Math. Gen. 37 (2004) 7567-7580.

[62] C. Cacciapuoti, R. Carlone, R. Figari, Decoherence induced by scattering: a three-dimensional model, J. Phys. A: Math. Gen. 38 (2005) 4933-4946.

[63] R. Adami, R. Figari, D. Finco, A. Teta, On the asymptotic dynamics of a quantum system composed by heavy and light particles, J. Phys. A: Math. Gen. 268 (2006) 819-852.

[64] S. Stenholm, Occurrences, observations and measurements in quantum mechanics, Phys. Scr. 47 (1993) $724-731$.

[65] K. Hornberger, Monitoring approach to open quantum dynamics using scattering theory, EPL 77 (2007) 50007.

[66] K. Hornberger, Open quantum dynamics via environmental monitoring, J. Phys.: Conf. Ser. 67 (2007) 012002.

[67] K. Jacobs, D. A. Steck, A straightforward introduction to continuous quantum measurement, Contemp. Phys. 47 (2006) 279-303.

[68] A. Barchielli, M. Gregoratti, Quantum Trajectories and Measurements in Continuous Time, Vol. 782 of Lecture Notes in Physics, Springer, Berlin, 2009.

[69] R. Dümcke, The low density limit for an N-level system interacting with a free Bose or Fermi gas, Commun. Math. Phys. 97 (1985) 331-359.

[70] G. Raffelt, G. Sigl, L. Stodolsky, Non-Abelian Boltzmann equation for mixing and decoherence, Phys. Rev. Lett. 70 (1993) $2363-2366$.

[71] S. Tsonchev, P. Pechukas, Binary collision model for quantum Brownian motion, Phys. Rev. E 61 (2000) 6171-6182.

[72] M. Kleckner, A. Ron, Decoherence of a pointer by a gas reservoir, Phys. Rev. A 63 (2001) 022110.

[73] M. Hellmich, Alicki's model of scattering-induced decoherence derived from Hamiltonian dynamics, J. Phys. A: Math. Gen. 37 (2004) 8711-8719.

[74] A. N. Pechen, Quantum stochastic equation for a test particle interacting with a dilute Bose gas, J. Math. Phys. 45 (2004) 400-417.

[75] A. N. Pechen, White noise approach to the low density limit of a quantum particle in a gas, in: M. Schuermann, U. Franz (Eds.), QP-PQ:Quantum Probability and White Noise Analysis, Vol. 18, World Scientific, Singapore, 2005 , p. 428.

[76] S. M. Barnett, J. D. Cresser, Quantum theory of friction, Phys. Rev. A 72 (2005) 022107.

[77] S. L. Adler, Normalization of collisional decoherence: squaring the delta function, and an independent cross-check, J. Phys. A: Math. Gen. 39 (2006) 14067-14074.

[78] J. J. Halliwell, Two derivations of the master equation of quantum Brownian motion, J. Phys. A: Math. Theor. 40 (2007) 3067-3080.

[79] A. Dominguez-Clarimon, A particle across a medium: How decoherence relates to the index of refraction., Ann. Phys. 322 (2007) 2085-2103. 
[80] F. Mintert, E. J. Heller, Simulation of open quantum systems, arXiv:0803.3883

[81] D. Benedetto, F. Castella, R. Esposito, M. Pulvirenti, Some considerations on the derivation of the nonlinear quantum Boltzmann equation, J. Stat. Phys. 116 (2004) 381-410.

[82] D. Benedetto, F. Castella, R. Esposito, M. Pulvirenti, Some considerations on the derivation of the nonlinear quantum Boltzmann equation II: the low density regime, J. Stat. Phys. 124 (2006) 951-996.

[83] D. Benedetto, F. Castella, R. Esposito, M. Pulvirenti, From the N-body Schrödinger equation to the quantum Boltzmann equation: a term-by-term convergence result in the weak coupling regime, Commun. Math. Phys. 277 (2008) 1-44.

[84] R. Steinigeweg, H.-P. Breuer, J. Gemmer, Transition from diffusive to ballistic dynamics for a class of finite quantum models, Phys. Rev. Lett. 99 (2007) 150601.

[85] M. Kadiroglu, J. Gemmer, Boltzmann-equation approach to transport in finite modular quantum systems, Phys. Rev. B 76 (2007) 024306.

[86] A. Kossakowski, On quantum statistical mechanics of non-Hamiltonian systems, Rep. Math. Phys. 3 (1972) $247-274$.

[87] A. D. Manita, Properties of translation-invariant quantum-dynamical semigroups, Theor. Math. Phys. 89 (1991) 12711281.

[88] D. D. Botvich, V. A. Malyshev, A. D. Manita, Translation invariant quantum master equation, Helv. Phys. Acta 64 (1991) 1072-1092.

[89] A. S. Holevo, A note on covariant dynamical semigroups, Rep. Math. Phys. 32 (1993) 211-216.

[90] A. S. Holevo, On conservativity of covariant dynamical semigroups, Rep. Math. Phys. 33 (1993) 95-110.

[91] A. S. Holevo, On translation-covariant quantum Markov equations., Izv. Math. 59 (1995) 427-443.

[92] A. S. Holevo, Covariant quantum Markovian evolutions, J. Math. Phys. 37 (1996) 1812-1832.

[93] B. Vacchini, Covariant mappings for the description of measurement, dissipation and decoherence in quantum mechanics, in E. Brünning, F. Pettrucione (Eds.) Theoretical Foundations of Quantum Information Processing and Communication, Lecture Notes in Physics 878, Springer-Verlag, Berlin, 2010, to appear; eprint arXiv:quant-ph/0707.0603.

[94] K. Huang, Statistical Mechanics, John Wiley \& Sons, New York, 1987.

[95] H. Spohn, J. L. Lebowitz, Irreversible thermodynamics for quantum systems weakly coupled to thermal reservoirs, Adv. Chem. Phys. 38 (1978) 109-142.

[96] G. Lindblad, Non-Equilibrium Entropy and Irreversibility, Reidel Publishing Company, Dordrecht, 1983.

[97] R. Alicki, M. Fannes, Quantum dynamical systems, Oxford University Press, Oxford, 2001.

[98] A. Wehrl, General properties of entropy, Rev. Mod. Phys. 50 (1978) 221-260.

[99] R. S. Ingarden, A. Kossakowski, M. Ohya, Information dynamics and open systems, Kluwer Academic Publishers, Dordrecht, 1997.

[100] B. Vacchini, K. Hornberger, Relaxation dynamics of a quantum Brownian particle in an ideal gas, Eur. Phys. J. ST 151 (2007) 59-72.

[101] H.-P. Breuer, B. Vacchini, Three-dimensional Monte Carlo simulations of the quantum linear Boltzmann equation, Phys. Rev. E 76 (2007) 036706.

[102] L. Diósi, Stochastic pure state representation for open quantum systems, Phys. Lett. 114A (1986) 451-454.

[103] C. W. Gardiner, A. S. Parkins, P. Zoller, Wave-function quantum stochastic differential equations and quantum-jump simulation methods, Phys. Rev. A 46 (1992) 4363-4381.

[104] H. Carmichael, An Open Systems Approach to Quantum Optics, Springer, Berlin, 1993.

[105] K. Molmer, Y. Castin, J. Dalibard, Monte Carlo wave-function method in quantum optics, J. Opt. Soc. Am. B 10 (1993) $524-538$.

[106] B. Vacchini, Quantum and classical features in the explanation of collisional decoherence, J. Mod. Opt. 51 (2004) 10251029.

[107] D. T. Gillespie, Markov Processes, Academic Press, Boston, 1992.

[108] H. Grabert, P. Schramm, G. Ingold, Quantum Brownian motion: The functional integral approach, Phys. Rep. 168 (1988) $115-207$.

[109] G. Ingold, Path integrals and their application to dissipative quantum systems, in: A. Buchleitner, K. Hornberger (Eds.), Coherent Evolution in Noisy Environments, Lecture Notes in Physics 611, Springer, Berlin, 2002, pp. 1-53.

[110] P. Hänggi, G. Ingold, Fundamental aspects of quantum Brownian motion, Chaos 15 (2005) 026105.

[111] U. Weiss, Quantum Dissipative Systems, 3rd Edition, World Scientific, Singapore, 2008.

[112] J. W. S. Rayleigh, Dynamical problems in illustration of the theory of gases, Phil. Mag. 32 (1891) 424-445.

[113] M. S. Green, Brownian motion in a gas of noninteracting molecules, J. Chem. Phys. 19 (1951) 1036-1046.

[114] C. S. W. Chang, G. E. Uhlenbeck, The kinetic theory of gases, in: J. D. Boer (Ed.), Studies in statistical mechanics, Vol. 5, North-Holland, Amsterdam, 1970, Ch. V.

[115] L. Ferrari, An improved differential form of the Boltzmann collision operator for a Rayleigh gas (or Brownian particles), Physica A 142 (1987) 441-466.

[116] V. Ambegaokar, Quantum Brownian motion and its classical limit, Ber. Bunsenges. Phys. Chem. 95 (1991) $400-404$.

[117] P. Pechukas, Quantum Brownian Motion, in: W. Gans, A. Blumen, A. Amann (Eds.), Large Scale Molecular Systems, Vol. 258 of NATO ASI Series, Plenum Press, New York, 1991, p. 123.

[118] A. Bassi, E. Ippoliti, B. Vacchini, On the energy increase in space-collapse models, J. Phys. A: Math. Gen. 38 (2005) $8017-8038$

[119] A. O. Caldeira, A. J. Leggett, Path integral approach to quantum Brownian motion, Physica A 121 (1983) 587-616. 
[120] A. O. Caldeira, A. J. Leggett, Quantum tunnelling in a dissipative system, apny 149 (1983) 374-456.

[121] A. J. Leggett, S. Chakravarty, A. T. Dorsey, M. P. A. Fisher, A. Garg, W. Zwerger, Dynamics of the dissipative two-state system, Rev. Mod. Phys. 59 (1987) 1-85.

[122] A. Barchielli, Continual measurements for quantum open systems, Nuovo Cimento B 74 (1983) 113-138.

[123] A. Sandulescu, H. Scutaru, Open quantum systems and the damping of collective modes in deep inelastic collisions, Ann. Physics 173 (1987) 277-317.

[124] A. Isar, Uncertainty, entropy and decoherence of the damped harmonic oscillator in the Lindblad theory of open quantum systems, Fortschr. Phys. 47 (1999) 855-879.

[125] B. Vacchini, Quantum optical versus quantum Brownian motion master equation in terms of covariance and equilibrium properties, J. Math. Phys. 43 (2002) 5446-5458.

[126] A. Isar, A. Sandulescu, H. Scutaru, E. Stefanescu, W. Scheid, Open quantum systems, Int. J. Mod. Phys. E 3 (1994) $635-714$.

[127] K. Jacobs, A Monte Carlo method for modeling thermal damping: Beyond the Brownian motion master equation, EPL 85 (2009) 40002.

[128] L. Diósi, On high temperature Markovian equation for quantum Brownian motion, Europhys. Lett. 22 (1993) 1-3.

[129] L. Diósi, Caldeira-Leggett master equation and medium temperatures, Physica A 199 (1993) 517-526.

[130] B. Vacchini, Non-abelian linear Boltzmann equation and quantum correction to Kramers and Smoluchowski equation, Phys. Rev. E 66 (2002) 027107.

[131] V. F. Sears, Neutron Optics, Oxford University Press, Oxford, 1989.

[132] S. A. Werner, H. Rauch, Neutron Interferometry, Oxford University Press, Oxford, 2000.

[133] C. S. Adams, M. Siegel, J. Mlynek, Atom optics, Phys. Rep. 240 (1994) 143-210.

[134] A. Miffre, M. Jacquey, M. Büchner, G. Trénec, J. Vigué, Atom interferometry, Phys. Scr. 74 (2006) C15-C23.

[135] M. Arndt, K. Hornberger, Quantum interferometry with complex molecules, in: Quantum Coherence in Solid State Systems, Proceedings of the International School of Physics "Enrico Fermi", Course CLXXI, Società Italiana di Fisica, 2009, (to appear), arXiv:0903.1614

[136] J. Vigue, Index of refraction of dilute matter in atomic interferometry, Phys. Rev. A 52 (1995) 3973-3975.

[137] H. Rauch, W. Treimer, U. Bonse, Test of a single crystal neutron interferometer, Phys. Lett. A 47 (1974) 369-371.

[138] J. R. Taylor, Scattering Theory, John Wiley \& Sons, New York, 1972.

[139] C. Champenois, Interférométrie atomique avec l'atome de lithium: analyse théorique et construction d'un interféromètre, applications., Ph.D. thesis, Université Paul Sabatier, Toulouse, available at http://tel.archives-ouvertes.fr/tel-00003602 (1999).

[140] C. Champenois, M. Jacquey, S. Lepoutre, M. Buchner, G. Trenec, J. Vigue, Index of refraction of gases for matter waves: Effect of the motion of the gas particles on the calculation of the index, Phys. Rev. A 77 (2008) 013621.

[141] R. C. Forrey, L. You, V. Kharchenko, A. Dalgarno, Index of refraction of noble gases for sodium matter waves, Phys. Rev. A 54 (1996) 2180-2184.

[142] V. Kharchenko, A. Dalgarno, Refractive index for matter waves in ultracold gases, Phys. Rev. A 63 (2001) 023615.

[143] T. D. Roberts, A. D. Cronin, D. A. Kokorowski, D. E. Pritchard, Glory oscillations in the index of refraction for matter waves, Phys. Rev. Lett. 89 (2002) 200406.

[144] S. N. Sanders, F. Mintert, E. J. Heller, Coherent scattering from a free gas, Phys. Rev. A 79 (2009) 023610.

[145] W. H. Zurek, Decoherence and the transition from quantum to classical, Phys. Today 44 (1991) 36-44.

[146] E. Joos, H. D. Zeh, C. Kiefer, D. Giulini, J. Kupsch, I.-O. Stamatescu, Decoherence and the Appearance of a Classical World in Quantum Theory, 2nd Edition, Springer, Berlin, 2003.

[147] W. H. Zurek, Decoherence, einselection, and the quantum origins of the classical, Rev. Mod. Phys. 75 (2003) $715-775$.

[148] M. Schlosshauer, Decoherence and the Quantum-to-Classical Transition, Springer-Verlag, Berlin, 2007.

[149] K. Hornberger, Introduction to decoherence theory, in: A. Buchleitner, C. Viviescas, M. Tiersch (Eds.), Entanglement and Decoherence, Lecture Notes in Physics 768, Springer, Berlin, 2009, pp. 221-276.

[150] B. Vacchini, Master-equations for the study of decoherence, Int. J. Theor. Phys. 44 (2005) 1011-1021.

[151] K. Hornberger, J. E. Sipe, M. Arndt, Theory of decoherence in a matter mave Talbot-Lau interferometer, Phys. Rev. A 70 (2004) 053608.

[152] W. Feller, An introduction to probability theory and its applications. Vol. II, John Wiley \& Sons Inc., New York, 1971.

[153] L. Lukacs, Characteristic Functions, Griffin, London, 1966.

[154] B. Vacchini, Theory of decoherence due to scattering events and Lévy processes, Phys. Rev. Lett. 95 (2005) 230402.

[155] H. Uys, J. D. Perreault, A. D. Cronin, Matter-wave decoherence due to a gas environment in an atom interferometer, Phys. Rev. Lett. 95 (2005) 150403.

[156] A. Ruiz, J. Breton, J. Gomez Llorente, Scattering cross-sections for low-energy rare-gas $+\mathrm{C}_{60}$ and $\mathrm{C}_{60}+\mathrm{C}_{60}$ collisions, Chem. Phys. Lett. 270 (1997) 121-128.

[157] G. C. Maitland, M. Rigby, E. B. Smith, W. A. Wakeham, Intermolecular Forces - Their Origin and Determination, Clarendon Press, Oxford, 1981.

[158] C.-C. Cheng, M. G. Raymer, Long-range saturation of spatial decoherence in wave-field transport in random multiplescattering media, Phys. Rev. Lett. 82 (1999) 4807-4810.

[159] T. Pfau, S. Spälter, C. Kurtsiefer, C. Ekstrom, J. Mlynek, Loss of spatial coherence by a single spontaneous emission, Phys. Rev. Lett. 73 (1994) 1223-1226. 
[160] M. S. Chapman, T. D. Hammond, A. Lenef, J. Schmiedmayer, R. A. Rubenstein, E. Smith, D. E. Pritchard, Photon scattering from atoms in an atom interferometer: Coherence lost and regained, Phys. Rev. Lett. 75 (1995) 3783 - 3787.

[161] D. A. Kokorowski, A. D. Cronin, T. D. Roberts, D. E. Pritchard, From single- to multiple-photon decoherence in an atom interferometer, Phys. Rev. Lett. 86 (2001) 2191-2195.

[162] L. Hackermüller, K. Hornberger, B. Brezger, A. Zeilinger, M. Arndt, Decoherence of matter waves by thermal emission of radiation, Nature 427 (2004) 711-714.

[163] K. Hornberger, S. Gerlich, H. Ulbricht, L. Hackermüller, S. Nimmrichter, I. V. Goldt, O. Boltalina, M. Arndt, Theory and experimental verification of Kapitza-Dirac Talbot-Lau interferometry, New J. Phys. 11 (2009) 043032.

[164] P. Facchi, Thermal decoherence in mesoscopic interference, J. Mod. Opt. 51 (2004) 1049-1055.

[165] K. Hornberger, L. Hackermüller, M. Arndt, Influence of molecular temperature on the coherence of fullerenes in a near-field interferometer, Phys. Rev. A 71 (2005) 023601.

[166] K. Hornberger, Thermal limitation of far-field matter-wave interference, Phys. Rev. A 73 (2006) 052102.

[167] S. Nimmrichter, K. Hornberger, H. Ulbricht, M. Arndt, Absolute absorption spectroscopy based on molecule interferometry, Phys. Rev. A 78 (2008) 063607.

[168] A. Viale, M. Vicari, N. Zanghi, Analysis of the loss of coherence in interferometry with macromolecules, Phys. Rev. A 68 (2003) 063610.

[169] F. C. Lombardo, F. D. Mazzitelli, P. I. Villar, Decoherence induced by a fluctuating Aharonov-Casher phase, Phys. Rev. A 72 (2005) 042111.

[170] P. Villar, F. Lombardo, Visibility fringe reduction due to noise-induced effects: Microscopic approach to interference experiments, Int. J. Mod. Phys. B 21 (2007) 4659-4676.

[171] T. Qureshi, A. Venugopalan, Decoherence and matter wave interferometry, Int. J. Mod. Phys. B 22 (2008) 981-990.

[172] R. Rubenstein, D. A. Kokorowski, A. Dhirani, T. Roberts, S. Gupta, J. Lehner, W. Smith, E. Smith, H. Bernstein, D. Pritchard, Measurement of the density matrix of a longitudinally modulated atomic beam, Phys. Rev. Lett. 83 (1999) $2285-2288$.

[173] R. Rubenstein, A. Dhirani, D. Kokorowski, T. Roberts, E. Smith, W. W. Smith, H. Bernstein, J. Lehner, S. Gupta, D. Pritchard, Search for off-diagonal density matrix elements for atoms in a supersonic beam, Phys. Rev. Lett. 82 (1999) 2018-2021.

[174] J. Anglin, J. Paz, W. Zurek, Deconstructing decoherence, Phys. Rev. A 55 (1997) 4041-4053.

[175] D. Kusnezov, A. Bulgac, G. D. Dang, Quantum Lévy processes and fractional kinetics, Phys. Rev. Lett. 82 (1999) 11361139.

[176] E. Lutz, Anomalous Lévy decoherence, Phys. Lett. A 293 (2002) 123-128.

[177] H. Schomerus, E. Lutz, Nonexponential decoherence and momentum subdiffusion in a quantum Lévy kicked rotator, Phys. Rev. Lett. 98 (2007) 260401.

[178] B. Bellomo, S. M. Barnett, J. Jeffers, Frictional quantum decoherence, J. Phys. A: Math. Theor. 40 (2007) $9437-9453$.

[179] F. M. Ramazanoglu, Approach to thermal equilibrium in the Caldeira-Leggett model, eprint arXiv:0812:2520v1.

[180] E. P. Wigner, Review of quantum mechanical measurement problem, in: P. Meystre, M. O. Scully (Eds.), Quantum Optics, Experimental Gravity and Measurement Theory, Plenum Press, New York, 1983, p. 43.

[181] A. Einstein, Über die von der molekularkinetischen Theorie der Wärme geforderte Bewegung von in ruhenden Flüssigkeiten suspendierten Teilchen, Ann. Phys. (Leipzig) 322 (1905) 549-560.

[182] C. Gocke, G. Röpke, Localization of coherent wave packets in plasmas due to decoherence, Contrib. Plasma Phys. 47 (2007) 291-296.

[183] C. Gocke, G. Röpke, Master equation of the reduced statistical operator of an atom in a plasma, Theor. Math. Phys. 154 (2008) $26-51$.

[184] S. Lovesey, Theory of neutron scattering from condensed matter. Vol.1. Nuclear scattering, Clarendon Press, Oxford, UK, 1984.

[185] F. Schwabl, Advanced quantum mechanics, Springer, New York, 2003.

[186] L. Pitaevskii, S. Stringari, Bose-Einstein condensation, Oxford University Press, Oxford, 2003.

[187] L. Lanz, B. Vacchini, Subdynamics of relevant observables: a field theoretical approach, Int. J. Modern Phys. A 17 (2002) 435-463.

[188] W. Brenig, Statistical theory of heat, Springer-Verlag, Berlin, 1989.

[189] L. Van Hove, Correlations in space and time and Born approximation scattering in systems of interacting particles, Phys. Rev. 95 (1954) 249-262.

[190] R. Alicki, S. Kryszewski, Completely positive Bloch-Boltzmann equations, Phys. Rev. A 68 (2003) 013809.

[191] S. Kryszewski, J. Czechowska, Positivity of Bloch-Boltzmann equations, Phys. Rev. A 74 (2006) 022719.

[192] S. G. Rautian, A. M. Shalagin, Kinetic Problems of Non-Linear Spectroscopy, North-Holland, Amsterdam, 1991.

[193] B. Vacchini, Non-Markovian dynamics for bipartite systems, Phys. Rev. A 78 (2008) 022112.

[194] A. A. Budini, Random Lindblad equations from complex environments, Phys. Rev. E 72 (2005) 056106.

[195] A. A. Budini, Lindblad rate equations, Phys. Rev. A 74 (2006) 053815.

[196] H.-P. Breuer, J. Gemmer, M. Michel, Non-Markovian quantum dynamics: Correlated projection superoperators and Hilbert space averaging, Phys. Rev. E 73 (2006) 016139.

[197] H.-P. Breuer, Non-Markovian generalization of the Lindblad theory of open quantum systems, Phys. Rev. A 75 (2007) 022103.

[198] M. Abramowitz, I. Stegun, Handbook of Mathematical Functions, Dover Publications, New York, 1965. 